\title{
Detecting kinematic boundary surfaces in phase space: particle mass measurements in SUSY-like events
}

\author{
Dipsikha Debnath, ${ }^{a}$ James S. Gainer, ${ }^{b}$ Can Kilic, ${ }^{c}$ Doojin Kim, ${ }^{a, d}$ \\ Konstantin T. Matchev ${ }^{a}$ and Yuan-Pao Yang ${ }^{c}$ \\ ${ }^{a}$ Physics Department, University of Florida, \\ Gainesville, FL 32611, U.S.A. \\ ${ }^{b}$ Department of Physics and Astronomy, University of Hawaii, \\ Honolulu, HI 96822, U.S.A. \\ ${ }^{c}$ Theory Group, Department of Physics and Texas Cosmology Center, \\ The University of Texas at Austin, Austin, TX 78712, U.S.A. \\ ${ }^{d}$ Theory Division, CERN, \\ CH-1211 Geneva 23, Switzerland \\ E-mail: dipsikha.debnath@gmail.com, jgainer@hawaii.edu, \\ kilic@physics.utexas.edu, immworry@gmail.com, \\ konstantin.matchev@gmail.com, yjp1986@utexas.edu
}

ABSTRACT: We critically examine the classic endpoint method for particle mass determination, focusing on difficult corners of parameter space, where some of the measurements are not independent, while others are adversely affected by the experimental resolution. In such scenarios, mass differences can be measured relatively well, but the overall mass scale remains poorly constrained. Using the example of the standard SUSY decay chain $\tilde{q} \rightarrow \tilde{\chi}_{2}^{0} \rightarrow \tilde{\ell} \rightarrow \tilde{\chi}_{1}^{0}$, we demonstrate that sensitivity to the remaining mass scale parameter can be recovered by measuring the two-dimensional kinematical boundary in the relevant three-dimensional phase space of invariant masses squared. We develop an algorithm for detecting this boundary, which uses the geometric properties of the Voronoi tessellation of the data, and in particular, the relative standard deviation (RSD) of the volumes of the neighbors for each Voronoi cell in the tessellation. We propose a new observable, $\bar{\Sigma}$, which is the average RSD per unit area, calculated over the hypothesized boundary. We show that the location of the $\bar{\Sigma}$ maximum correlates very well with the true values of the new particle masses. Our approach represents the natural extension of the one-dimensional kinematic endpoint method to the relevant three dimensions of invariant mass phase space.

Keywords: Supersymmetry Phenomenology

ARXiv EPrint: 1611.04487 


\section{Contents}

1 Introduction 1

2 Endpoint formulas and partitioning of parameter space 10

$\begin{array}{ll}2.1 \text { Notation and conventions } & 10\end{array}$

$\begin{array}{lll}2.2 & \text { Endpoint formulas } & 10\end{array}$

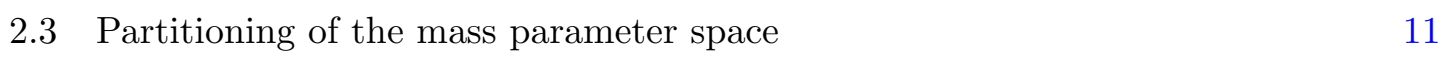

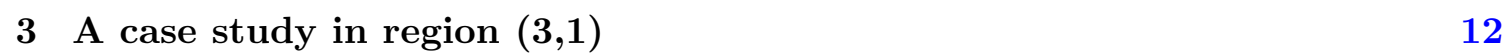

$\begin{array}{lll}3.1 & \text { Kinematical properties along the flat direction } & 12\end{array}$

3.2 A toy study with uniformly distributed background 22

$\begin{array}{lll}3.3 & \text { A study with } t \bar{t} \text { dilepton background events } & 25\end{array}$

4 A case study in region $(3,2) \quad 28$

4.1 Kinematical properties along the flat direction 28

4.2 A toy study with uniformly distributed background 33

4.3 A study with $t \bar{t}$ dilepton background events 34

$\begin{array}{lll}4.4 & \text { A detector level study } & 36\end{array}$

$\begin{array}{lll}4.5 & \boldsymbol{D} \text {-pair production and combinatorics effects } & 37\end{array}$

$\begin{array}{llr}5 & \text { Conclusions } & 39\end{array}$

$\begin{array}{ll}\text { A Inverse formulas } & 40\end{array}$

\section{Introduction}

The dark matter problem is currently our best experimental evidence for the existence of new particles and interactions beyond the Standard Model (BSM). A great number of ongoing experiments are trying to discover dark matter particles through direct [1] or indirect detection [2]. In principle, dark matter particles could also be produced in high energy collisions at the Large Hadron Collider (LHC) at CERN, providing a complementary discovery probe in a controlled experimental environment [3].

Since the dark matter particles must be stable on cosmological timescales, in many popular BSM models they carry some conserved quantum number. The simplest choice is a $\mathbb{Z}_{2}$ parity, which is known as $R$-parity in models with low energy supersymmetry (SUSY) [4], Kaluza-Klein (KK) parity in models with universal extra dimensions (UED) [5], T-parity in Little Higgs models [6], etc. As a result, the dark matter particles are necessarily produced in pairs: either directly, or in the cascade decays of other, heavier BSM particles [7]. The prototypical such cascade decay is shown in figure 1, in which a new particle $D$ undergoes a series of two-body decays, terminating in the dark matter candidate $A$, which is neutral 


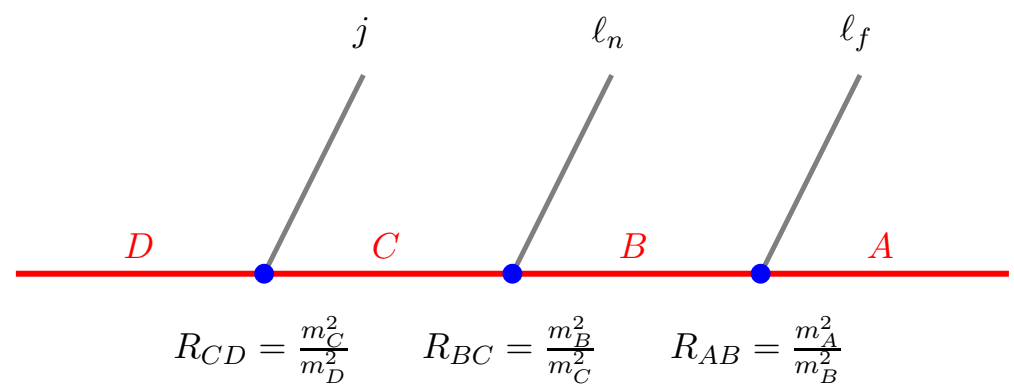

Figure 1. The generic decay chain under consideration in this paper: $D \rightarrow j C \rightarrow j \ell_{n} B \rightarrow j \ell_{n} \ell_{f} A$, where $A, B, C$ and $D$ are new BSM particles, while the SM decay products consist of one jet $j$ and two leptons, labelled "near" $\ell_{n}$ and "far" $\ell_{f}$. In the SUSY case, $D$ represents a squark $\tilde{q}$, $C$ is a heavier neutralino $\tilde{\chi}_{2}^{0}, B$ is a charged slepton $\tilde{\ell}$ and $A$ is the lightest neutralino $\tilde{\chi}_{1}^{0}$, which escapes undetected. The masses of the BSM particles are denoted by $m_{D}, m_{C}, m_{B}$ and $m_{A}$. The corresponding ratios of squared masses $R_{C D}, R_{B C}$ and $R_{A B}$ are introduced for convenience in writing the kinematic endpoint formulas (2.4)-(2.11) and delineating the relevant regions in the mass parameter space (1.1) (see also eq. (2.3) and figure 2 below).

and stable, and thus escapes undetected. Under those circumstances, measuring the set of four masses

$$
\left\{m_{D}, m_{C}, m_{B}, m_{A}\right\}
$$

is a difficult problem, which has been attracting a lot of attention over the last 20 years (for a review, see [8]). The main challenge stems from the fact that the momentum of particle $A$ is not measured, so that the standard technique of directly reconstructing the new particles as invariant mass resonances does not apply. Instead, one has to somehow infer the new masses (1.1) from the measured kinematic distributions of the visible SM decay products.

In the decay chain of figure 1 , the SM decay products are taken to be a quark jet $j$ and two leptons, labelled "near" $\ell_{n}$ and "far" $\ell_{f}$. This choice is motivated by the following arguments:

- At a hadron collider like the LHC, strong production dominates, thus particle $D$ is very likely to be colored. At the same time, the dark matter candidate $A$ is neutral, therefore the color must be shed somewhere along the decay chain in the form of a QCD jet. Here we assume that this "color-shedding" occurs in the $D \rightarrow C$ transition, ${ }^{1}$ since one expects the strong decays of particle $D$ to be the dominant ones.

- The presence of leptons among the SM decay products in figure 1 is theoretically not guaranteed, but is nevertheless experimentally motivated. First, leptonic signatures have significantly lower SM backgrounds and thus represent clean discovery channels.

\footnotetext{
${ }^{1}$ We note that in principle one can test this assumption experimentally, e.g. by constructing suitably defined on-shell constrained $M_{2}$ variables corresponding to the competing event topologies [9], or by studying the shapes and the correlations for the invariant mass variables considered below [10]. Such an exercise is useful, but beyond the scope of this paper.
} 
Second, the momentum of a lepton is measured much better than that of a jet, therefore the masses (1.1) will be measured with a better precision in a leptonic channel (as opposed to a purely jetty channel). Finally, if the SM decay products in figure 1 were all jets, in light of the arising combinatorial problem [11-16], we would have to resort to sorted invariant mass variables [17, 18], whose kinematic endpoints are less pronounced and thus more difficult to measure over the SM backgrounds.

- From a historical perspective, the best motivation for considering the decay chain of figure 1 is that it is ubiquitous in SUSY, where $D$ represents a squark $\tilde{q}, C$ is a heavier neutralino $\tilde{\chi}_{2}^{0}, B$ is a charged slepton $\tilde{\ell}$ and $A$ is the lightest neutralino $\tilde{\chi}_{1}^{0}$, which escapes the detector and leads to missing transverse energy $E_{T}$. In the two most popular frameworks of SUSY breaking, gravity-mediated and gauge-mediated, the combination of (a) specific high scale boundary conditions, and (b) renormalization group evolution of the soft SUSY parameters down to the weak scale, leads to just the right mass hierarchy for the decay chain of figure 1 to occur. In the late 1990's and early 2000's, this prompted a flurry of activity on the topic of mass determination in such "SUSY-like" missing energy events. Soon afterwards, it was also realized that the decay chain of figure 1 is not exclusive to supersymmetry, but the same final state signature also appears in other models, e.g. minimal UED [19] and littlest Higgs [20].

To date, a large variety of mass measurement techniques for SUSY-like events have been developed. Roughly speaking, they all can be divided into two categories.

- Exclusive methods. In this case, one takes advantage of the presence of two decay chains in the event (they are often assumed identical) and the available $E_{T}$ measurement. Several approaches are then possible. For example, in the so-called "polynomial methods" one attempts to solve explicitly for the momenta of the invisible particles in a given event, possibly using additional information from prior measurements of kinematic endpoints [21-35]. ${ }^{2}$ Alternatively, utilizing information from both branches, one could introduce suitable transverse ${ }^{3}$ variables whose distributions exhibit an upper kinematic endpoint indicative of the parent particle mass [48-63]. In the latter case, one still retains a residual dependence on the unknown dark matter particle mass $m_{A}$, which must be fixed by some other means, e.g. via the kink method [64-71] or by performing a sufficient number of independent measurements $[53,57]$. While they could be potentially quite sensitive, these exclusive methods are also less robust, since they rely on the correct identification of all objects in the event, and are thus prone to combinatorial ambiguities, the effects from $E_{T}$ resolution, initial and final state radiation, underlying event and pileup, etc.

\footnotetext{
${ }^{2}$ For long enough decay chains, the polynomial methods are able to solve for the invisible momenta, even without additional experimental input and without a second decay chain in the event. If the decay chain of figure 1 contained an additional two-body decay to a visible particle, just 5 events are sufficient for solving the event kinematics [22, 27].

${ }^{3}$ Transversality is not strictly necessary, in fact it may even be beneficial to work with $3+1$-dimensional variants of those variables [36-47].
} 
- Inclusive methods. In this case, one focuses on the decay chain from figure 1 itself, disregarding what else is going on in the event. Using only the measured momenta of the visible SM decay products, i.e., the jet and the two leptons, one could form all possible invariant mass combinations, ${ }^{4}$ namely $m_{\ell \ell}, m_{j \ell_{n}}, m_{j \ell_{f}}$, and $m_{j \ell \ell}$, measure their respective upper kinematic endpoints

$$
\left\{m_{\ell \ell}^{\max }, m_{j \ell_{n}}^{\max }, m_{j \ell_{f}}^{\max }, m_{j \ell \ell}^{\max }\right\},
$$

and use them to solve for the four input parameters (1.1). As just described, this approach is too naive, as it overlooks the remaining combinatorial problem involving the two leptons $\ell_{n}$ and $\ell_{f}$. Since "near" and "far" cannot be distinguished on an event by event basis, the variables $m_{j \ell_{n}}$ and $m_{j \ell_{f}}$ are ill defined. This is why it has become customary to redefine the two jet-lepton invariant mass combinations $\operatorname{as}^{5}$

$$
\begin{aligned}
& m_{j l(\mathrm{lo})} \equiv \min \left\{m_{j l_{n}}, m_{j l_{f}}\right\}, \\
& m_{j l(\text { hi })} \equiv \max \left\{m_{j l_{n}}, m_{j l_{f}}\right\} .
\end{aligned}
$$

The distributions of the newly defined quantities (1.3) and (1.4) also exhibit upper kinematic endpoints, $m_{j l(\mathrm{lo})}^{\max }$ and $m_{j l(\mathrm{hi})}^{\max }$, respectively. Then, instead of (1.2), one can use the new well-defined set of measurements

$$
\left\{m_{l l}^{\max }, m_{j l l}^{\max }, m_{j l(\mathrm{lo})}^{\max }, m_{j l(\mathrm{hi})}^{\max }\right\}
$$

to invert and solve for the input mass parameters (1.1). This procedure constitutes the classic kinematic endpoint method for mass measurements, which has been successfully tested for several SUSY benchmark points [77-84].

However, despite its robustness and simplicity, the kinematic endpoint method still has a couple of weaknesses. As we show below, taken together, they essentially lead to an almost flat direction in the solution space, thus jeopardizing the uniqueness of the mass determination. The first of these two problems is purely theoretical - it is well known that in certain regions of the parameter space (1.1) the four measurements (1.5) are not independent, but obey the relation [81]

$$
\left(m_{j l l}^{\max }\right)^{2}=\left(m_{j l(\mathrm{hi})}^{\max }\right)^{2}+\left(m_{l l}^{\max }\right)^{2} .
$$

In practice, this means that the measurements (1.5) fix only three out of the four mass parameters (1.1), leaving one degree of freedom undetermined. In what follows, we shall choose to parametrize this "flat direction" with the mass $m_{A}$ of the lightest among the four new particles $D, C, B$ and $A$. One can then use, e.g., the first three of the measurements in (1.5) and solve uniquely for the three heavier masses $m_{D}, m_{C}$, and $m_{B}$, leaving $m_{A}$

\footnotetext{
${ }^{4}$ In general, one is not limited to Lorentz-invariant variables only, e.g., recently it was suggested to study the peak of the energy distribution as a measure of the mass scale [72-75].

${ }^{5} \mathrm{~A}$ more recent alternative approach is to introduce new invariant mass variables which are symmetric functions of $m_{j \ell_{n}}$ and $m_{j \ell_{f}}$, thus avoiding the need to distinguish $\ell_{n}$ from $\ell_{f}$ on an event per event basis [76].
} 
as a free parameter. We list the relevant inversion formulas in appendix A. The obtained one-parameter family of mass spectra

$$
\left\{\begin{array}{l}
m_{D}=m_{D}\left(m_{A} ; m_{l l}^{\max }, m_{j l l}^{\max }, m_{j l(\mathrm{lo})}^{\max }\right), \\
m_{C}=m_{C}\left(m_{A} ; m_{l l}^{\max }, m_{j l l}^{\max }, m_{j l(\mathrm{lo})}^{\max }\right), \\
m_{B}=m_{B}\left(m_{A} ; m_{l l}^{\max }, m_{j l l}^{\max }, m_{j l(\mathrm{lo})}^{\max }\right), \\
m_{A}
\end{array}\right.
$$

will satisfy the three measured kinematic endpoints $m_{l l}^{\max }, m_{j l l}^{\max }$, and $m_{j l(\mathrm{lo})}^{\max }$ by construction. What is more, in parameter space regions where eq. (1.6) holds, the family (1.7) will also obey the fourth measurement of $m_{j l(\mathrm{hi})}^{\max }$, so that the four measurements (1.5) will be insufficient to lift the $m_{A}$ degeneracy in (1.7).

These considerations beg the following two questions, which will be addressed in this paper.

1. In the remaining part of the parameter space, where (1.6) does not hold and $m_{j l(\text { hi }}^{\max }$ provides an independent fourth measurement, how well is the $m_{A}$ degeneracy lifted after all? With the explicit examples of sections 3 and 4 below, we shall show that although in theory the additional measurement of $m_{j l(\mathrm{hi})}^{\max }$ determines the value of $m_{A}$, in practice this may be difficult to achieve, since the effect is very small and will be swamped by the experimental resolution.

2. In the region of parameter space in which (1.6) holds, what additional measurement should be used, and how well does it lift the degeneracy? In the existing literature,

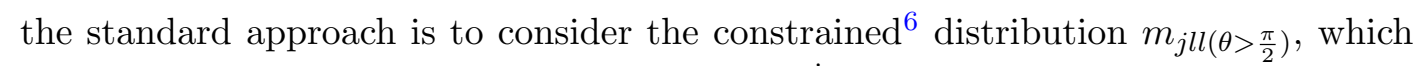
exhibits a useful lower kinematic endpoint $m_{j l l\left(\theta>\frac{\pi}{2}\right)}^{\min }[85,86]$. In what follows, we shall therefore always supplement the original set of 4 measurements (1.5) with the additional measurement of $m_{j l l\left(\theta>\frac{\pi}{2}\right)}^{\min }$ to obtain the extended set

$$
\left\{m_{l l}^{\max }, m_{j l l}^{\max }, m_{j l(\mathrm{lo})}^{\max }, m_{j l(\mathrm{hi})}^{\max }, m_{j l l\left(\theta>\frac{\pi}{2}\right)}^{\min }\right\},
$$

so that in principle there is sufficient information to determine the four unknown masses. Even then, we shall show that the sensitivity of the additional experimental input $m_{j l l\left(\theta>\frac{\pi}{2}\right)}^{\min }$ to the previously found flat direction (1.7) is very low. First of all, it is already well appreciated that the measurement of $m_{j l l\left(\theta>\frac{\pi}{2}\right)}^{\min }$ is very challenging, since in the vicinity of its lower endpoint, the shape of the signal distribution is concave

\footnotetext{
${ }^{6}$ The distribution $m_{j l l\left(\theta>\frac{\pi}{2}\right)}$ is nothing but the usual $m_{j l l}$ distribution taken over a subset of the original events, namely those which satisfy the additional dilepton mass constraint

$$
\frac{m_{l l}^{\max }}{\sqrt{2}}<m_{l l}<m_{l l}^{\max }
$$

In the rest frame of particle $B$, this cut implies the following restriction on the opening angle $\theta$ between the two leptons [86]

$$
\theta>\frac{\pi}{2}
$$

thus justifying the notation for $m_{j l l\left(\theta>\frac{\pi}{2}\right)}$.
} 
downward, which makes it difficult to extract the endpoint with simple linear fitting, and one has to use the whole shape of the $m_{j l l\left(\theta>\frac{\pi}{2}\right)}$ distribution [87]. Secondly, as we shall show in the examples below, the variation of the value of $m_{j l l\left(\theta>\frac{\pi}{2}\right)}^{\min }$ along the flat direction (1.7) can be numerically quite small, and therefore the sensitivity of the added fifth measurement along the flat direction (1.7) is not that great.

Either way, we see that the known methods for lifting the degeneracy of the flat direction (1.7) will face severe limitations once we take into account the experimental resolution, finite statistics, backgrounds, etc. [88, 89] Thus the first goal of this paper will be to illustrate the severity of the problem, i.e. to quantify the "flatness" of the family of solutions (1.7). For this purpose, we shall reuse the study points from ref. [89], which at the time were meant to illustrate discrete ambiguities, i.e. cases where two distinct points in mass parameter space (1.1) accidentally happen to give mathematically identical values for all five measurements (1.8). Here we shall extend those study points to a family of mass spectra (1.7) which give mathematically identical values for the first three ${ }^{7}$ of the measurements (1.8), and numerically very similar values for the remaining measurements.

Having identified the problem, the second goal of the paper is to propose a novel solution to it and investigate its viability. Our starting point is the observation that the signal events from the decay chain in figure 1 populate the interior of a compact region in the $\left(m_{j \ell_{n}}, m_{\ell \ell}, m_{j \ell_{f}}\right)$ space, whose boundary is given by the surface $\mathcal{S}$ defined by the constraint $[17,90,91]$

$$
\mathcal{S}: \quad \hat{m}_{j \ell_{f}}^{2}=\left[\sqrt{\hat{m}_{\ell \ell}^{2}\left(1-\hat{m}_{j \ell_{n}}^{2}\right)} \pm \frac{m_{B}}{m_{C}} \sqrt{\hat{m}_{j \ell_{n}}^{2}\left(1-\hat{m}_{\ell \ell}^{2}\right)}\right]^{2},
$$

which, for convenience, is written in terms of the unit-normalized variables

$$
\hat{m}_{j \ell_{n}}=\frac{m_{j \ell_{n}}}{m_{j \ell_{n}}^{\max }}, \quad \hat{m}_{\ell \ell}=\frac{m_{\ell \ell}}{m_{\ell \ell}}, \quad \hat{m}_{j \ell_{f}}=\frac{m_{j \ell_{f}}}{m_{j \ell_{f}}^{\max }} .
$$

We note that both the shape and the size of the surface $\mathcal{S}$ depend on the input mass spectrum (1.1), i.e., $\mathcal{S}\left(m_{A}, m_{B}, m_{C}, m_{D}\right)$, and this dependence is precisely what we will be targeting with our method to be described below.

In its traditional implementation, the kinematic endpoint method is essentially ${ }^{8}$ using the kinematic endpoints (1.2) of the one-dimensional projections of the signal population onto each of the three axes $m_{j \ell_{n}}, m_{\ell \ell}$ and $m_{j \ell_{f}}$, as well as onto the "radial" direction $m_{j \ell \ell}=$ $\sqrt{m_{j \ell_{n}}^{2}+m_{\ell \ell}^{2}+m_{j \ell_{f}}^{2}}$. This approach is suboptimal because it ignores correlations and misses endpoint features along the other possible projections. The only way to guarantee that we are using the full available information in the data is to fit to the three-dimensional boundary (1.9) itself [92, 93], which will be the approach advocated here. As previously observed in [92] (and extended to a broader class of event topologies in [94]), most of

\footnotetext{
${ }^{7}$ And sometimes four, if we are in parts of parameter space where (1.6) holds.

${ }^{8}$ The fact that one has to use $m_{j l(\mathrm{lo})}$ and $m_{j l(\mathrm{hi})}$ in place of $m_{j \ell_{n}}$ and $m_{j \ell_{f}}$ does not change the gist of the argument.
} 
the signal events are populated near the phase space boundary (1.9), on which the signal number density $\rho_{s}$ formally becomes singular. This fact is rather fortuitous, since it implies a relatively sharp change in the local number density as we move across the phase space boundary, even in the presence of SM backgrounds (with some number density $\rho_{b}$, which is expected to be a relatively smooth function). Thus, we need to develop a suitable method for identifying regions in phase space where the gradient of the total number density $\rho \equiv \rho_{b}+\rho_{s}$ is relatively large, and then fit to them the analytical parametrization (1.9) in order to obtain the best fit values for the four new particle masses (1.1).

The first step of this program was already accomplished in our earlier paper [93], building on the idea originally proposed in [95] for finding "edges" in two-dimensional stochastic distributions of point data. Ref. [95] suggested that interesting features in the data, e.g., edge discontinuities, kinks, and so on, can be identified by analyzing the geometric properties of the Voronoi tessellation (for a basic introduction to Voronoi tessellations, see [96]) of the data. ${ }^{9}$ The volume $v_{i}$ of a given Voronoi cell generated by a data point at some location $\vec{r}_{i}$ provides an estimate of the functional value of the number density $\rho$ at that location,

$$
\rho\left(\vec{r}_{i}\right) \sim \frac{1}{v_{i}}
$$

Therefore, in order to obtain an estimate of $|\vec{\nabla} \rho(\vec{r})|$, we can construct variables which compare the properties of the Voronoi cell and its direct neighbors. Among the different options investigated in refs. [95, 98], the relative standard deviation (RSD), $\bar{\sigma}_{i}$, of the volumes of neighboring cells, was identified as the most promising tagger of edge cells. The RSD was defined as follows. Let $N_{i}$ be the set of neighbors of the $i$-th Voronoi cell $C_{i}$, with volumes, $\left\{v_{j}\right\}$, for $j \in N_{i}$. The $\mathrm{RSD}, \bar{\sigma}_{i}$, is now defined by

$$
\bar{\sigma}_{i} \equiv \frac{1}{\left\langle v\left(N_{i}\right)\right\rangle} \sqrt{\sum_{j \in N_{i}} \frac{\left(v_{j}-\left\langle v\left(N_{i}\right)\right\rangle\right)^{2}}{\left|N_{i}\right|-1}},
$$

where we have normalized by the average volume of the set of neighbors, $N_{i}$, of the $i$-th cell

$$
\left\langle v\left(N_{i}\right)\right\rangle \equiv \frac{1}{\left|N_{i}\right|} \sum_{j \in N_{i}} v_{j}
$$

Subsequently, in [93] we showed that this procedure for tagging edge cells can be readily extended to three-dimensional point data, as is the case here. The end result of the method was a set of Voronoi cells which have been tagged as "edge cell candidates" since their values of $\bar{\sigma}_{i}$ were above the chosen threshold [93]. With the thus obtained set of edge cells in hand, it appears that we are in a perfect position to perform a mass measurement, simply by finding the set of values for $\left\{m_{A}, m_{B}, m_{C}, m_{D}\right\}$ which maximize the overlap between our tagged edge cells and the hypothesized surface $\mathcal{S}$. We have checked that this approach indeed works and gives a reasonable estimate of the true mass spectrum. However, here

\footnotetext{
${ }^{9}$ We note the existence of efficient codes for finding Voronoi tessellations in the form of the qHULL algorithms [97]. Wrappers that allow the use of these algorithms in many frameworks also exist, and in this work we use a private Python code to compute the geometric attributes of the Voronoi cells.
} 
we prefer to suggest a slightly modified alternative, which accomplishes the same goal, but with somewhat better precision.

The problem with fitting to a subset of the original data set (namely the set of Voronoi cells which happened to pass the $\bar{\sigma}_{i}$ cut) is that we are still throwing away useful information, e.g., the Voronoi cells which barely failed the cut. In spite of formally failing, those cells are nevertheless still quite likely to be edge cells. Thus, in order to retain the full amount of information in our data, we prefer to abandon this "cut and fit" approach, and instead design a global variable which is calculated over the full data set. The only requirement is that the variable is maximized (or minimized, as the case may be) for the true values of the masses $\left\{m_{A}, m_{B}, m_{C}, m_{D}\right\}$.

In order to motivate such a variable, consider for a moment the case when the function $\rho(\vec{r})$ is known analytically, then let us investigate the (normalized) surface integral

$$
\frac{\int_{\tilde{\mathcal{S}}\left(\tilde{m}_{A}, \tilde{m}_{B}, \tilde{m}_{C}, \tilde{m}_{D}\right)} d a|\vec{\nabla} \rho(\vec{r})|}{\int_{\tilde{\mathcal{S}}\left(\tilde{m}_{A}, \tilde{m}_{B}, \tilde{m}_{C}, \tilde{m}_{D}\right)} d a}
$$

for some arbitrary trial ${ }^{10}$ values $\left(\tilde{m}_{A}, \tilde{m}_{B}, \tilde{m}_{C}, \tilde{m}_{D}\right)$ of the unknown masses (1.1). The meaning of the quantity (1.14) is very simple: it is the average gradient of $\rho(\vec{r})$ over the chosen surface $\tilde{\mathcal{S}}$. We expect the dominant contributions to the integral to come from regions where the gradient is large, and we know that the gradient is largest on the true phase space boundary $\mathcal{S}\left(m_{A}, m_{B}, m_{C}, m_{D}\right)$, defined in terms of the true values of the particle masses. However, if our choice for $\left(\tilde{m}_{A}, \tilde{m}_{B}, \tilde{m}_{C}, \tilde{m}_{D}\right)$ is wrong, the integration surface $\tilde{\mathcal{S}}$ will be far from the true phase space boundary $\mathcal{S}$, and those large contributions will be missed. The only way to capture all of the large contributions to the integral is to have $\tilde{\mathcal{S}}$ coincide with the true $\mathcal{S}$, and this is only possible if in turn the trial masses are exactly equal to the true particle masses. This suggests a method of mass measurement whereby the true mass spectrum is obtained as the result of an optimization problem involving the quantity (1.14).

Of course, in our case the analytical form of the integrand $|\vec{\nabla} \rho(\vec{r})|$ is unknown, but we can obtain a closely related quantity using the Voronoi tessellation of the data. Following $[93,95]$, we shall utilize the RSD $\bar{\sigma}_{i}$ defined in (1.12), which has been shown to be a good indicator of edge cells, and replace the integrand in (1.14) as

$$
|\vec{\nabla} \rho(\vec{r})| \longrightarrow g(\vec{r}) \equiv \bar{\sigma}_{i} \text { for } \vec{r} \in C_{i} .
$$

In other words, the gradient estimator ${ }^{11}$ function $g(\vec{r})$ is defined so that it is equal to the RSD $\bar{\sigma}_{i}$ of the Voronoi cell $C_{i}$ in which the point $\vec{r}$ happens to be. Eqs. (1.14) and (1.15) suggest that the variable which we should be maximizing is

$$
\bar{\Sigma}\left(\tilde{m}_{A}, \tilde{m}_{B}, \tilde{m}_{C}, \tilde{m}_{D}\right) \equiv \frac{\int_{\tilde{\mathcal{S}}\left(\tilde{m}_{A}, \tilde{m}_{B}, \tilde{m}_{C}, \tilde{m}_{D}\right)} d a g(\vec{r})}{\int_{\tilde{\mathcal{S}}\left(\tilde{m}_{A}, \tilde{m}_{B}, \tilde{m}_{C}, \tilde{m}_{D}\right)} d a} .
$$

\footnotetext{
${ }^{10}$ From here on trial values for the masses will carry a tilde to distinguish from the true values of the masses which will have no tilde. Correspondingly, $\tilde{\mathcal{S}}$ stands for a hypothesized "trial" boundary surface (1.9) obtained with trial values of the mass parameters.

${ }^{11}$ Note that $g(\vec{r})$ is not supposed to be an approximation for $|\vec{\nabla} \rho(\vec{r})|$, the crucial property for us is that the two functions peak in the same location.
} 
It obviously depends on our choice of trial masses $\left(\tilde{m}_{A}, \tilde{m}_{B}, \tilde{m}_{C}, \tilde{m}_{D}\right)$, and as argued above, we expect the maximum of $\bar{\Sigma}$ to occur for the correct choice $\left(m_{A}, m_{B}, m_{C}, m_{D}\right)$, i.e.

$$
\max _{\tilde{m}_{A}, \tilde{m}_{B}, \tilde{m}_{C}, \tilde{m}_{D}} \bar{\Sigma}\left(\tilde{m}_{A}, \tilde{m}_{B}, \tilde{m}_{C}, \tilde{m}_{D}\right) \simeq \bar{\Sigma}\left(m_{A}, m_{B}, m_{C}, m_{D}\right)
$$

This hypothesis will be tested and validated with explicit examples below in sections 3 and 4.

The paper is organized as follows. In the next section 2 we shall first review the well known formulas for the one-dimensional kinematic endpoints (1.8) and introduce the corresponding relevant partitioning of the mass parameter space into domain regions. In the next two sections we shall concentrate on the two most troublesome regions, $(3,2)$ and $(3,1)$, where the problematic relationship (1.6) holds. We shall pick one study point in each region, then study how well our conjecture (1.17) is able to determine the true mass spectrum. In principle, (1.17) involves optimization over 4 continuous variables, which is very time consuming (additionally, we have to perform the integration in the numerator of (1.16) by Monte Carlo). This is why for simplicity we choose to illustrate the power of our method with a one-dimensional toy study along the problematic flat direction (1.7). In particular, for each of our two study points we shall assume that the first three kinematic endpoints $m_{l l}^{\max }, m_{j l l}^{\max }$, and $m_{j l(\mathrm{lo})}^{\max }$ are already measured, leaving us only the task of determining the remaining degree of freedom $m_{A}$ along the flat direction defined in (1.7). Correspondingly, we shall consider the whole family of mass spectra (1.7) which passes through a given study point. This family will eventually take us into the neighboring parameter space regions, including the third potentially problematic region, namely $(2,3)$, in which (1.6) is satisfied. For each family, we shall perform the following investigations

- As a warm up, we shall first illustrate that for each of the three distributions, $m_{l l}$, $m_{j l l}$, and $m_{j l(\mathrm{lo})}$, the endpoint along the flat direction is the same (as expected by construction).

- We shall then investigate the variation of the kinematic endpoint of the $m_{j l(h i)}$ distribution along the flat direction (1.7). The endpoint value $m_{j l(\mathrm{hi})}^{\max }$ is expected to be constant in regions $(3,2),(3,1)$ and $(2,3)$, so the main question will be, how much does it vary in the remaining parameter space regions.

- We shall similarly investigate the variation of the lower kinematic endpoint $m_{j l l\left(\theta>\frac{\pi}{2}\right)}^{\min }$ along the flat direction (1.7). Together with the previous item, this will serve as an illustration of the main weakness of the classic kinematic endpoint method for mass measurements.

- Then we shall illustrate the distortion of the kinematic boundary surface (1.9) along the flat direction (1.7). The size of the distortion will be indicative of the precision with which one can hope to perform the mass measurement (1.17) using the kinematic boundary surface in phase space. 
- Finally, we shall perform the fitting (1.17) along the flat direction parameterized by $\tilde{m}_{A}$. We shall show results in two cases: (a) when the background events are distributed uniformly in $m^{2}$ phase space, and (b) when the background is coming from dilepton $t \bar{t}$ events.

We shall summarize and conclude in section 5. Appendix A contains the inversion formulas needed to define the flat direction (1.7).

\section{Endpoint formulas and partitioning of parameter space}

\subsection{Notation and conventions}

Following [89], we introduce for convenience some shorthand notation for the mass squared ratios

$$
R_{i j} \equiv \frac{m_{i}^{2}}{m_{j}^{2}}
$$

where $i, j \in\{A, B, C, D\}$. Note that in (2.1) there are only three independent quantities, which can be taken to be the set $\left\{R_{A B}, R_{B C}, R_{C D}\right\}$. To save writing, we will also introduce convenient shorthand notation for the five kinematic endpoints as follows

$$
a=\left(m_{l l}^{\max }\right)^{2}, \quad b=\left(m_{j l l}^{\max }\right)^{2}, \quad c=\left(m_{j l(\mathrm{lo})}^{\max }\right)^{2}, \quad d=\left(m_{j l(\mathrm{hi})}\right)^{2}, \quad e=\left(m_{j l l\left(\theta>\frac{\pi}{2}\right)}\right)^{2} .
$$

Note that these represent the kinematic endpoints of the mass squared distributions. ${ }^{12}$

In the next two sections we shall use the three endpoint measurements $m_{l l}^{\max }, m_{j l l}^{\max }$, and $m_{j l(\mathrm{lo})}^{\max }$ to fix $m_{D}, m_{C}$ and $m_{B}$, leaving $m_{A}$ as a free parameter. Another way to think about this procedure is to note that the parameter space (1.1) can be equivalently parametrized as

$$
\left\{R_{C D}, R_{B C}, R_{A B}, m_{A}\right\} .
$$

Then, the endpoint measurements of $m_{l l}^{\max }, m_{j l l}^{\max }$, and $m_{j l(\mathrm{lo})}^{\max }$ can be used to fix the ratios $R_{C D}, R_{B C}$ and $R_{A B}$ (see appendix $\mathrm{A}$ ), leaving the overall mass scale undetermined and parametrized by $m_{A}$.

\subsection{Endpoint formulas}

The kinematical endpoints are given by the following formulas:

$$
\begin{aligned}
& a \equiv\left(m_{l l}^{\max }\right)^{2}=m_{D}^{2} R_{C D}\left(1-R_{B C}\right)\left(1-R_{A B}\right) ; \\
& b \equiv\left(m_{j l l}^{\max }\right)^{2}=\left\{\begin{array}{lll}
m_{D}^{2}\left(1-R_{C D}\right)\left(1-R_{A C}\right), & \text { for } R_{C D}<R_{A C}, & \text { case }(1,-), \\
m_{D}^{2}\left(1-R_{B C}\right)\left(1-R_{A B} R_{C D}\right), & \text { for } R_{B C}<R_{A B} R_{C D}, & \text { case }(2,-), \\
m_{D}^{2}\left(1-R_{A B}\right)\left(1-R_{B D}\right), & \text { for } R_{A B}<R_{B D}, & \text { case }(3,-), \\
m_{D}^{2}\left(1-\sqrt{R_{A D}}\right)^{2}, & \text { otherwise, } & \text { case }(4,-) ;
\end{array}\right.
\end{aligned}
$$

\footnotetext{
${ }^{12}$ Contrast to the notation of ref. [81], which uses $a, b, c, d$ to label the same endpoints, but for the linear masses.
} 


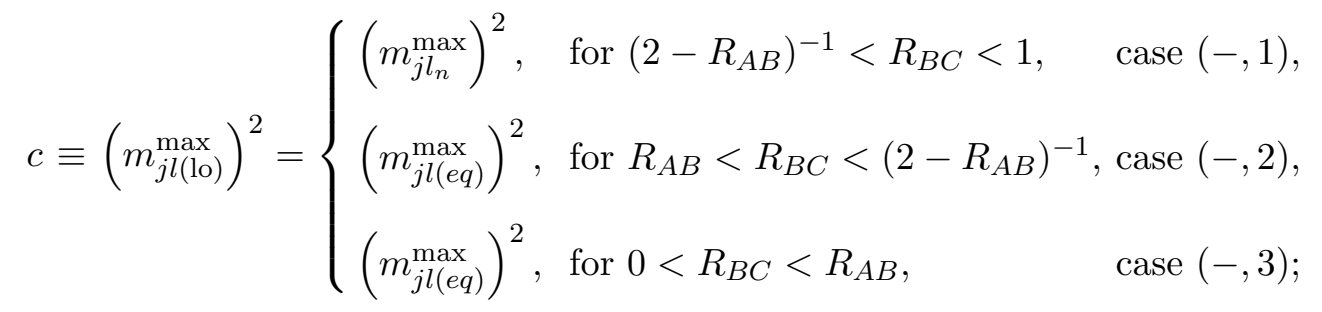

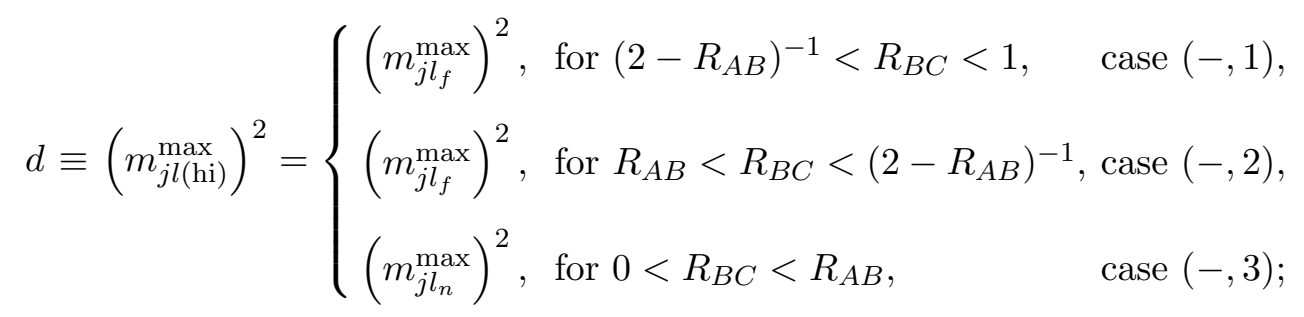

where

$$
\begin{aligned}
\left(m_{j l_{n}}^{\max }\right)^{2} & =m_{D}^{2}\left(1-R_{C D}\right)\left(1-R_{B C}\right) \\
\left(m_{j l_{f}}^{\max }\right)^{2} & =m_{D}^{2}\left(1-R_{C D}\right)\left(1-R_{A B}\right) \\
\left(m_{j l(e q)}^{\max }\right)^{2} & =m_{D}^{2}\left(1-R_{C D}\right)\left(1-R_{A B}\right)\left(2-R_{A B}\right)^{-1} .
\end{aligned}
$$

Finally, the endpoint $m_{j l l\left(\theta>\frac{\pi}{2}\right)}^{\min }$ introduced earlier in the Introduction, is given by

$$
\begin{aligned}
e \equiv\left(m_{j l l\left(\theta>\frac{\pi}{2}\right)}^{\min }\right)^{2}= & \frac{1}{4} m_{D}^{2}\left\{\left(1-R_{A B}\right)\left(1-R_{B C}\right)\left(1+R_{C D}\right)\right. \\
& \left.+2\left(1-R_{A C}\right)\left(1-R_{C D}\right)-\left(1-R_{C D}\right) \sqrt{\left(1+R_{A B}\right)^{2}\left(1+R_{B C}\right)^{2}-16 R_{A C}}\right\}
\end{aligned}
$$

\subsection{Partitioning of the mass parameter space}

One can see that the formulas (2.5)-(2.7) are piecewise-defined: they are given in terms of different expressions, depending on the parameter range for $R_{C D}, R_{B C}$ and $R_{A B}$. This divides the $\left\{R_{C D}, R_{B C}, R_{A B}\right\}$ parameter subspace from (2.3) into several distinct regions, illustrated in figure 2. Following [81], we label those by a pair of integers $\left(N_{j l l}, N_{j l}\right)$. As already indicated in eqs. (2.5)-(2.7), the first integer $N_{j l l}$ identifies the relevant case for $m_{j l l}^{\max }$, while the second integer $N_{j l}$ identifies the corresponding case for $\left(m_{j l(\mathrm{lo})}^{\max }, m_{j l(\mathrm{hi})}^{\max }\right)$. One can show that only 9 out of the 12 pairings $\left(N_{j l l}, N_{j l}\right)$ are physical, and they are all exhibited within the unit square of figure 2. In what follows, an individual study point within a given region $\left(N_{j l l}, N_{j l}\right)$ will be marked with corresponding subscripts as $P_{N_{j l l} N_{j l}}$.

Using (2.4), (2.5) and (2.7), it is easy to check that the "bad" relation (1.6), which can be equivalently rewritten in the new notation as

$$
b=a+d,
$$

is identically satisfied in regions $(3,1),(3,2)$ and $(2,3)$ of figure 2 . Therefore, as already discussed, in these regions one would necessarily have to rely on the additional information provided by the measurement of the $e$ endpoint (2.11). 


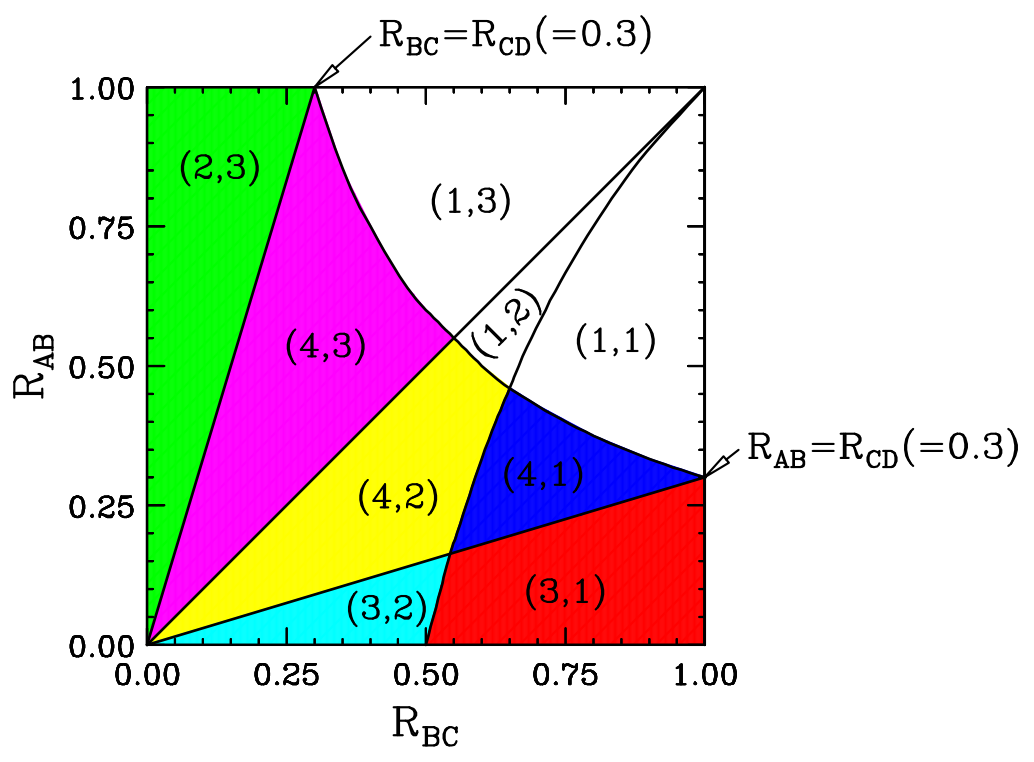

Figure 2. A slice through the $\left\{R_{C D}, R_{B C}, R_{A B}\right\}$ parameter space at a fixed $R_{C D}=0.3$. The $\left(R_{B C}, R_{A B}\right)$ plane exhibits the nine definition domains $\left(N_{j l l}, N_{j l}\right)$ of the set of equations $(2.5)-(2.7)$. For the purposes of this paper, only six of those regions will be in play, and we have color-coded them as follows: region $(3,1)$ in red, region $(4,1)$ in blue, region $(3,2)$ in cyan, region $(4,2)$ in yellow, region $(4,3)$ in magenta, and region $(2,3)$ in green.

Before concluding this rather short preliminary section, we direct the reader's attention to the color-coding in figure 2 , where we have shaded in color six of the parameter space regions: region $(3,1)$ in red, region $(4,1)$ in blue, region $(3,2)$ in cyan, region $(4,2)$ in yellow, region $(4,3)$ in magenta, and region $(2,3)$ in green. It will turn out that the two families of mass spectra considered in the next two sections will visit the six color-shaded regions. For the benefit of the reader, in the remainder of the paper we shall strictly adhere to this color scheme - for example, results obtained for a study point from a particular region will always be plotted with the color of the respective region: study points in region $(3,1)$ are red, study points in region $(4,1)$ are blue, etc.

\section{A case study in region $(3,1)$}

\subsection{Kinematical properties along the flat direction}

In this section we shall study the flat direction (1.7) in mass parameter space which is generated by a study point $P_{31}$ from region $(3,1)$ (the same study point was used in [89] for a slightly different purpose). Table 1 lists some relevant information for the study point $P_{31}$ : the input mass spectrum (1.1), the corresponding mass squared ratios (2.1), and the predicted kinematic endpoints (1.8), also reminding the reader of the alternative shorthand notation (2.2). As discussed in the Introduction, starting from the point $P_{31}$, we can follow a one-dimensional trajectory (1.7) through the parameter space (2.3) so that everywhere along the trajectory the prediction for the three endpoints $a, b$ and $c$ is unchanged (see figure 6 below). This trajectory is illustrated in figure 3, where we show its projections 


\begin{tabular}{|c|c|c|c|c|c|}
\hline & \multicolumn{2}{|c|}{ true branch } & \multicolumn{2}{|c|}{ auxilliary branch } \\
\hline \multicolumn{2}{|l|}{ Region } & $(3,1)$ & $4,1)$ & $(4,3)$ & $(2,3)$ \\
\hline \multicolumn{2}{|l|}{ Study point } & $P_{31}$ & $P_{41}$ & $P_{43}$ & $P_{23}$ \\
\hline \multicolumn{2}{|l|}{$m_{A}(\mathrm{GeV})$} & 236.64 & 5000.00 & $2,000.00$ & 100.00 \\
\hline \multicolumn{2}{|l|}{$m_{B}(\mathrm{GeV})$} & 374.16 & 5126.02 & 2040.56 & 124.78 \\
\hline \multicolumn{2}{|l|}{$m_{C}(\mathrm{GeV})$} & 418.33 & 5168.03 & 2167.36 & 272.54 \\
\hline \multicolumn{2}{|l|}{$m_{D}(\mathrm{GeV})$} & 500.00 & 5256.90 & 2256.90 & 362.23 \\
\hline \multicolumn{2}{|l|}{$R_{A B}$} & 0.400 & 0.951 & 0.960 & 0.642 \\
\hline \multicolumn{2}{|l|}{$R_{B C}$} & 0.800 & 0.984 & 0.886 & 0.210 \\
\hline \multicolumn{2}{|l|}{$R_{C D}$} & 0.700 & 0.966 & 0.922 & 0.566 \\
\hline$m_{l l}^{\max }(\mathrm{GeV})$ & $\sqrt{a}$ & \multicolumn{4}{|c|}{144.91} \\
\hline$m_{j l l}^{\max }(\mathrm{GeV})$ & $\sqrt{b}$ & \multicolumn{4}{|c|}{256.90} \\
\hline$m_{j l(\mathrm{lo})}^{\max }(\mathrm{GeV})$ & $\sqrt{c}$ & \multicolumn{4}{|c|}{122.47} \\
\hline$m_{j l(\mathrm{hi})}^{\max }(\mathrm{GeV})$ & $\sqrt{d}$ & 212.13 & & 212.13 & 212.13 \\
\hline$m_{j l l\left(\theta>\frac{\pi}{2}\right)}(\mathrm{GeV})$ & $\sqrt{e}$ & 132.10 & 129.73 & 130.79 & 141.78 \\
\hline
\end{tabular}

Table 1. Mass spectrum and expected kinematic endpoints for the study point $P_{31}$ from region $(3,1)$ which was discussed in ref. [89], together with three additional study points illustrating the different regions from figure 2 encountered by the parameter space trajectories from figure 3 . By construction, all study points give identical values for the kinematic endpoints $m_{l l}^{\max }, m_{j l l}^{\max }$ and $m_{j l(\mathrm{lo})}^{\max }$. Furthermore, in accordance with $(1.6)$, the two study points $P_{31}$ and $P_{23}$ from regions $(3,1)$ and $(2,3)$ have identical values of $m_{j l(\mathrm{hi})}^{\mathrm{max}}$. The remaining two study points $P_{41}$ and $P_{43}$, representing regions $(4,1)$ and $(4,3)$, have essentially the same value for $m_{j l(\mathrm{hi})}^{\max }$ as well. The last row lists the predicted values for $m_{j l l\left(\theta>\frac{\pi}{2}\right)}^{\min }$, which are slightly different, and allow discriminating between the four endpoints in theory, but not in practice.

onto the three planes $\left(R_{B C}, R_{A B}\right)$ (left panel), $\left(R_{A B}, R_{C D}\right)$ (middle panel) and $\left(R_{B C}, R_{C D}\right)$ (right panel). The lines in figure 3 are parametrized by the continuous test mass parameter $\tilde{m}_{A}$. For any given fixed value of $\tilde{m}_{A}$, the trajectory in figure 3 predicts the test values for the other three mass parameters, namely $\tilde{m}_{B}, \tilde{m}_{C}$ and $\tilde{m}_{D}$. This is shown more explicitly in figure 4 , where we plot the mass differences $\tilde{m}_{B}-\tilde{m}_{A}$ (solid lines), $\tilde{m}_{C}-\tilde{m}_{A}$ (dashed lines), and $\tilde{m}_{D}-\tilde{m}_{A}$ (dotted lines), as a function of $\tilde{m}_{A}$. All lines in figures 3 and 4 are color-coded using the same color conventions as for the parameter space regions in figure 2. Initially, as we move away from point $P_{31}$ (marked with the red square in figure 3 ), we are still within the red region $(3,1)$, and the trajectory is therefore colored in red and parametrically given by eqs. (A.8)-(A.10). As the value of $\tilde{m}_{A}$ is reduced from its nominal value $(236.6 \mathrm{GeV})$ at the point $P_{31}$, the mass spectrum gets lighter and eventually we reach $\tilde{m}_{A}=0$, where (the red portion of) the trajectory terminates at $R_{A B}=0, R_{B C} \simeq 0.67$ and $R_{C D} \simeq 0.58$. If, on the other hand, we start increasing $\tilde{m}_{A}$ from its nominal $P_{31}$ value, the 

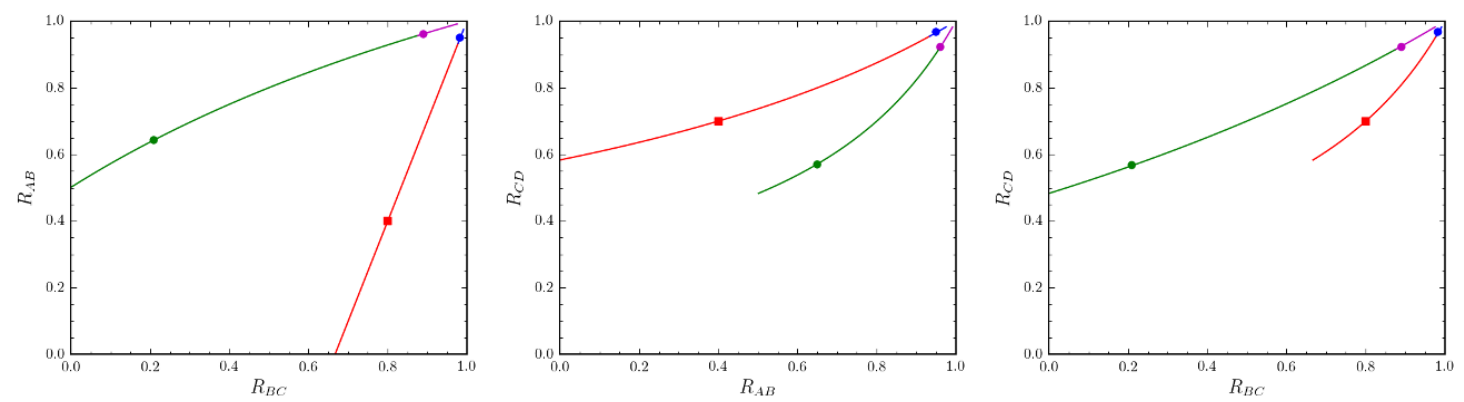

Figure 3. The two trajectories in mass parameter space leading to the same endpoints $a, b$ and $c$. The lines are colored in accordance with the coloring convention for the regions depicted in figure 2 . The red square marks the original study point $P_{31}$ from table 1 , while the circles denote the other three study points from table 1: $P_{41}$ in region $(4,1)$ (blue circle), $P_{43}$ in region $(4,3)$ (magenta circle), and $P_{23}$ in region $(2,3)$ (green circle).

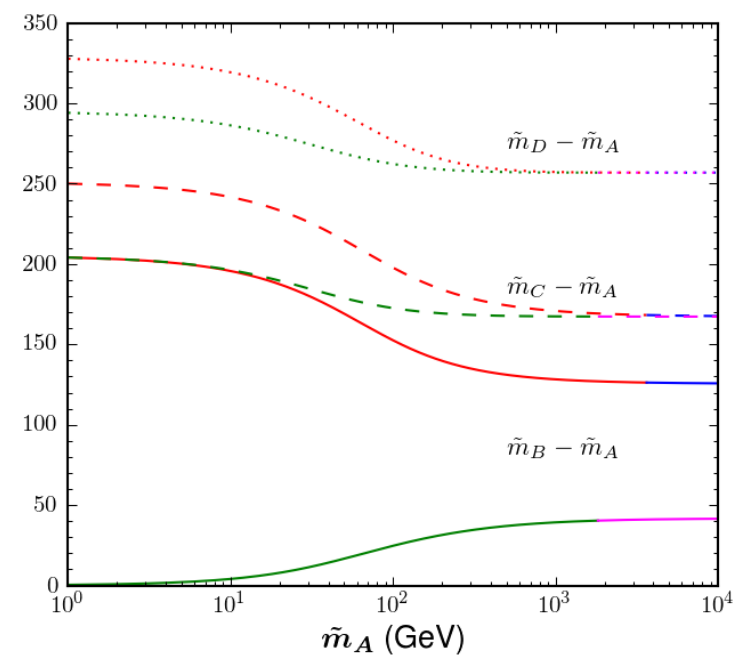

Figure 4. Mass spectra along the flat direction specified by the study point $P_{31}$. As a function of $\tilde{m}_{A}$, we plot the mass differences $\tilde{m}_{B}-\tilde{m}_{A}$ (solid lines), $\tilde{m}_{C}-\tilde{m}_{A}$ (dashed lines), and $\tilde{m}_{D}-\tilde{m}_{A}$ (dotted lines), which would preserve the values for the three kinematic endpoints $a, b$ and $c$.

spectrum gets heavier, and we start approaching the neighboring region $(4,1)$. Eventually, at around $\tilde{m}_{A} \sim 3600 \mathrm{GeV}$, the trajectory crosses into region $(4,1)$ and thus changes its color to blue. This transition is illustrated in the left panel of figure 5 , where we plot the mass squared ratios $R_{A B}, R_{B C}$ and $R_{C D}$ (solid lines), together with some other relevant quantities (dotted lines). In particular, the boundary between regions $(3,1)$ and $(4,1)$ is given by the relation $R_{A B}=R_{B D}$, see (A.2) and (A.29). We can see that crossover more clearly in the insert in the left panel of figure 5 , where the line color changes from red to blue as soon as the $R_{B D}$ (dotted) line crosses the $R_{A B}$ (solid) line.

Once we are in region $(4,1)$, we follow the blue portion of the trajectory in figure 3 , which is parametrically defined by eqs. (A.36)-(A.38). We choose a representative study point for region $(4,1)$ as well - it is denoted by $P_{41}$ and listed in the third (blue shaded) column of table 1 . The corresponding mass spectrum is clearly very heavy, but is nev- 

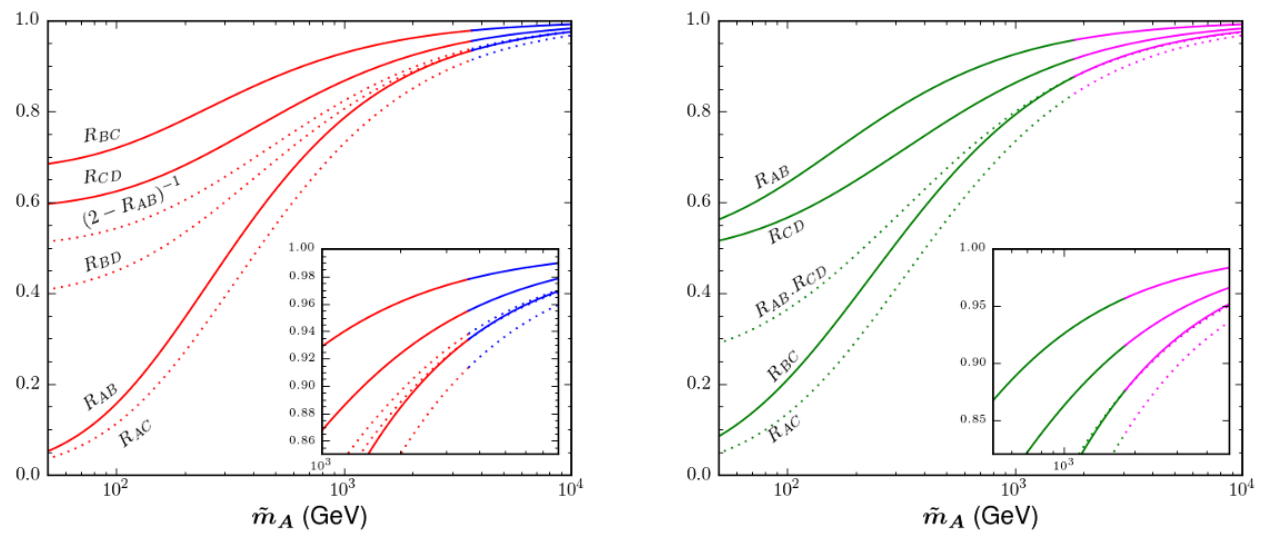

Figure 5. The equivalent representation of figure 4 in terms of the mass squared ratios $R_{A B}$, $R_{B C}$ and $R_{C D}$ (solid lines). The dotted lines depict various quantities of interest which are used to delineate the regions in figure 2. The left panel shows the true branch passing through regions $(3,1)$ (red) and $(4,1)$ (blue), while the right panel shows the auxiliary branch through regions $(2,3)$ (green) and $(4,3)$ (magenta). The left insert zooms in on the transition between regions $(3,1)$ and $(4,1)$ near $\tilde{m}_{A}=3600 \mathrm{GeV}$, while the right insert focuses on the transition between regions $(2,3)$ and $(4,3)$ near $\tilde{m}_{A}=1800 \mathrm{GeV}$.

ertheless perfectly consistent with the three measured endpoints $a, b$ and $c$, as shown in figure 6 . As seen in figure 3, the blue portion of the mass trajectory appears headed for the point $\left(R_{A B}, R_{B C}, R_{C D}\right)=(1,1,1)$, which is indeed reached in the limit of $\tilde{m}_{A} \rightarrow \infty$, without ever entering into the neighboring region $(1,1) .{ }^{13}$

Figure 3 reveals that the mass family (1.7) through our study point $P_{31}$ includes a segment which starts at $\left(R_{A B}, R_{B C}, R_{C D}\right)=(0,0.67,0.58)$ and ends at $\left(R_{A B}, R_{B C}, R_{C D}\right)=$ $(1,1,1)$, visiting regions $(3,1)$ and $(4,1)$. Since the actual study point $P_{31}$ belongs to this segment, in what follows we shall refer to it as "the true branch". However, figure 3 also shows that there is an additional disconnected segment of the mass trajectory through the green region $(2,3)$ and the magenta region $(4,3)$. In the following, we shall refer to this additional segment as "the auxiliary branch". Note that this terminology is introduced only for clarity and should not be taken too literally - as far as the measured endpoints $a, b$ and $c$ are concerned, all points on the true and auxiliary branches are on the same footing, since the experimenter would have no way of knowing a priori which is the true branch and which is the auxiliary branch. This is why we have to seriously consider points on the auxiliary branch as well. We choose two representative study points, which are listed in the last two columns of table 1: point $P_{43}$ belongs to the magenta region $(4,3)$, while point $P_{23}$ is in the green region $(2,3)$. As shown in figure 3 , the auxiliary branch starts at $\left(R_{A B}, R_{B C}, R_{C D}\right)=(0.5,0,0.48)$ and asymptotically meets the true branch at the corner point $\left(R_{A B}, R_{B C}, R_{C D}\right)=(1,1,1)$. The transition between the two regions $(2,3)$ and $(4,3)$ along the auxiliary branch is illustrated in the right panel of figure 5 . According to (A.21) and (A.54), the boundary between regions $(2,3)$ and $(4,3)$ is defined by

\footnotetext{
${ }^{13}$ Note that as the value of $R_{C D}$ increases, the $(1,1)$ region shrinks and for $R_{C D}=1$ it disappears altogether.
} 

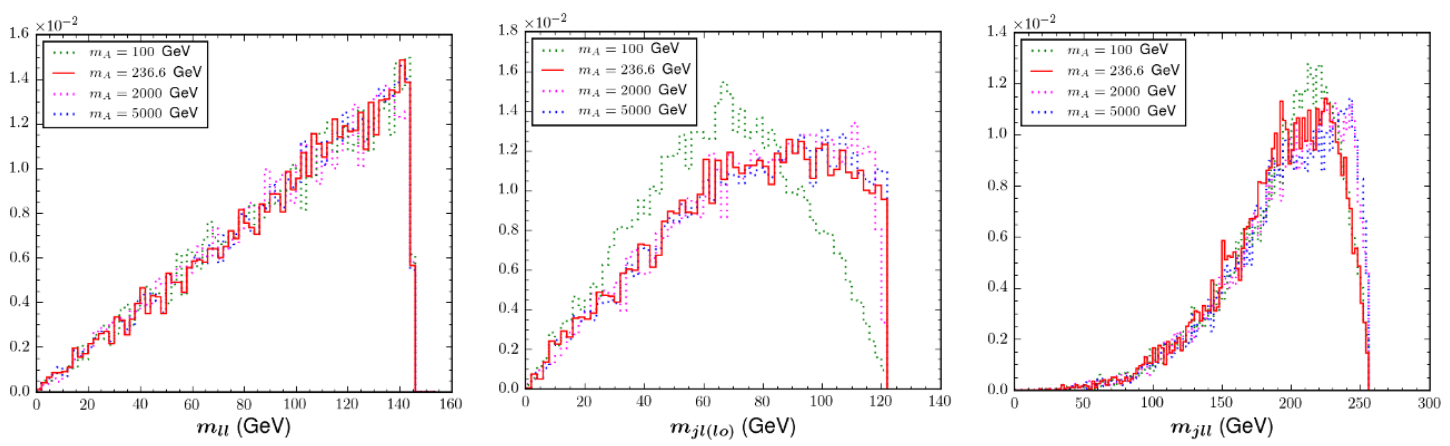

Figure 6. Unit-normalized invariant mass distributions for the four study points from table 1: the distribution of $m_{\ell \ell}$ (left panel), $m_{j \ell(1 \circ)}$ (middle panel), and $m_{j \ell \ell}$ (right panel). The lines are color coded according to our conventions from figure 2 and table 1: red for $P_{31}$, blue for $P_{41}$, magenta for $P_{43}$ and green for $P_{23}$.

the relation $R_{B C}=R_{A B} R_{C D}$. The right panel of figure 5 confirms this: the color of the auxiliary branch in figures 3 and 4 changes from green to magenta as soon as the dotted line representing the product $R_{A B} R_{C D}$ crosses the solid line for $R_{B C}$.

To summarize our discussion so far, we have imposed the three endpoint measurements $a, b$ and $c$ on the four-dimensional parameter space (1.1), reducing it to the one-dimensional parameter curve depicted in figures 3 and 4 . The curve consists of two branches which visit four of the colored regions in figure 2, and we have chosen one study point in each region. The four study points are listed in table 1, and their predicted invariant mass distributions from the ROOT phase space generator [99] are shown in figure 6: $m_{\ell \ell}$ in the left panel, $m_{j \ell(\mathrm{lo})}$ in the middle panel and $m_{j \ell \ell}$ in the right panel. By construction, for any points along the mass trajectory (1.7), and in particular for the four study points from table 1, these distributions share common kinematic endpoints. Furthermore, as figure 6 reveals, the shapes of most distributions are also very similar, which makes it difficult to pinpoint our exact location along the mass trajectory (1.7). This is why in the remainder of this section, we shall focus on the question, what additional measurements may allows us to discriminate experimentally points along the two branches in figures 3 and 4, and in particular distinguish between the four study points in table 1.

One obvious possibility is to investigate the remaining kinematic endpoints $d$ and $e$, which are analyzed in figures 7 and 8 , respectively. The left panels show the theoretical predictions for the kinematic endpoints $\sqrt{d}=m_{j \ell(\mathrm{hi})}^{\max }$ and $\sqrt{e}=m_{j l l\left(\theta>\frac{\pi}{2}\right)}^{\min }$ along the flat direction (1.7) as a function of $\tilde{m}_{A}$, while the right panels exhibit the corresponding invariant mass distributions for each of our four study points from table 1.

Let us first focus on figure 7 which illustrates the $\tilde{m}_{A}$ dependence of the $m_{j \ell(\text { hi) }}$ distribution and its kinematic endpoint $\sqrt{d}$. As we have already discussed, in regions $(3,1)$ and $(2,3)$ the additional measurement of $\sqrt{d}$ is not useful, since it is not independent the value of $d$ is predicted by the relation (2.12), as confirmed by the left panel in figure 7 , where the red and green dotted lines representing those two regions are perfectly flat and insensitive to $\tilde{m}_{A}$. However, this still leaves open the possibility that in the remaining two regions, namely $(4,1)$ and $(4,3)$, the measurement of the $d$ endpoint will be able to lift 

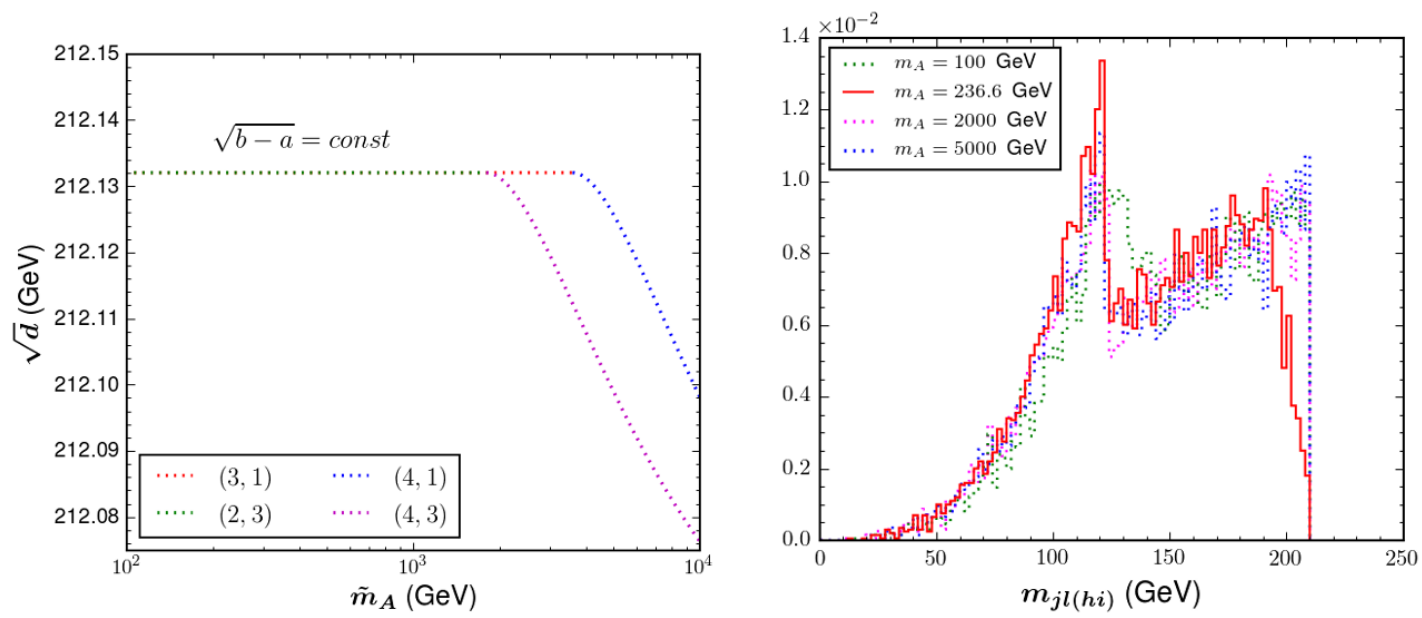

Figure 7. Left: the prediction for the kinematic endpoint $\sqrt{d}$ along the flat direction (1.7) generated by $P_{31}$, as a function of the trial value of the parameter $\tilde{m}_{A}$. Right: the same as figure 6 , but for the distribution $m_{j \ell(\mathrm{hi})}$.
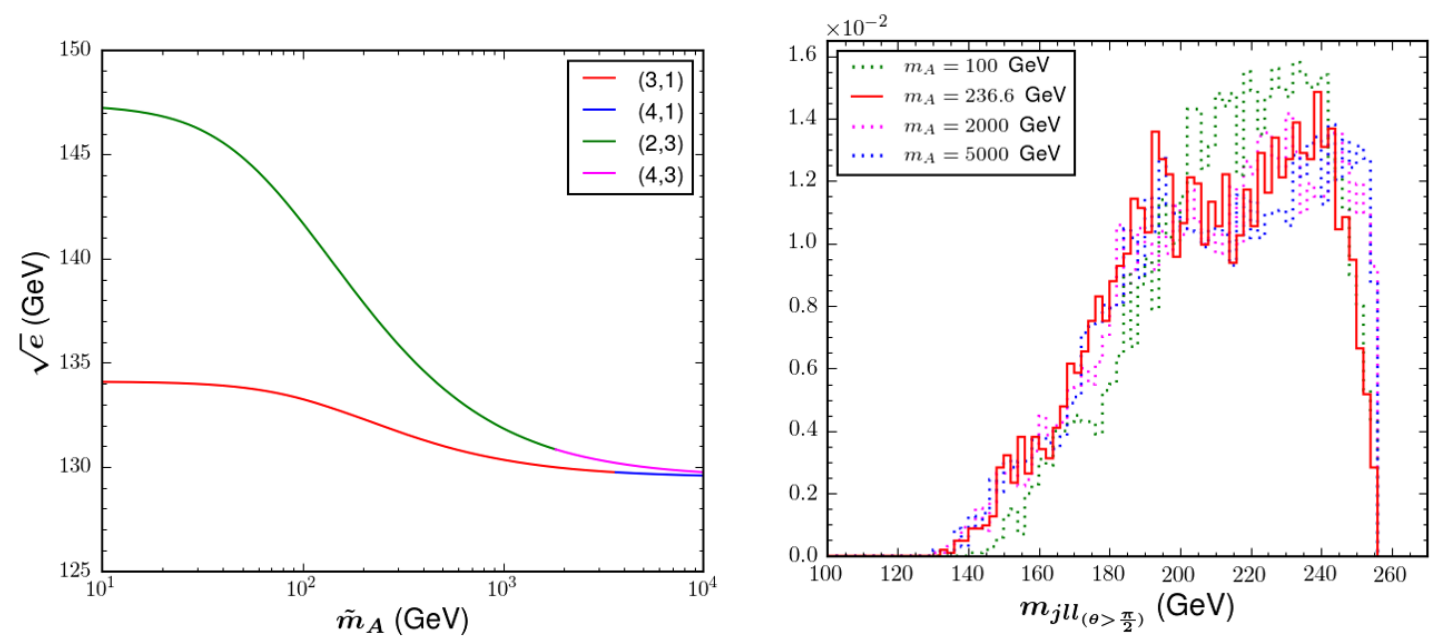

Figure 8. The same as figure 7 , but for the endpoint $\sqrt{e}$ and the corresponding distribution $m_{j l l\left(\theta>\frac{\pi}{2}\right)}$.

the degeneracy and determine the value of $m_{A}$, since, at least in theory, $d$ is a non-trivial function of $\tilde{m}_{A}$, see (A.39b) and (A.63b). Unfortunately, figure 7 demonstrates that this is not the case in practice - the $\tilde{m}_{A}$ dependence is extremely weak, and the endpoint value for $\sqrt{d}$ only changes by a few tens of $\mathrm{MeV}$ as $\tilde{m}_{A}$ is varied over a range of several $\mathrm{TeV}$ ! This lack of sensitivity is the reason why we have been referring to the family of mass spectra (1.7) as a "flat direction" in mass parameter space. Clearly, due to the finite experimental resolution, an endpoint measurement with a precision of tens of $\mathrm{MeV}$ is not feasible, the anticipated experimental errors at the LHC are significantly higher, on the order of a few GeV [100].

It is instructive to understand this lack of sensitivity analytically, by studying, e.g. the mathematical expression (A.39b) for $d$ which is relevant for region $(4,1)$. Figures 4 and 7 
already showed that region $(4,1)$ occurs at large values of $\tilde{m}_{A}$, where the spectrum is relatively heavy - on the order of several $\mathrm{TeV}$. At the same time, the measured parameter inputs into (A.39b), namely the endpoints $a, b$ and $c$, are all on the order of several hundred $\mathrm{GeV}$. This suggests an expansion in terms of $1 / \tilde{m}_{A}$ as

$$
d\left(a, b, c, \tilde{m}_{A}\right) \equiv K_{0}+\frac{K_{1}}{\tilde{m}_{A}}+\mathcal{O}\left(\frac{1}{\tilde{m}_{A}^{2}}\right) .
$$

Using (A.39b), we get the expansion coefficients to be

$$
\begin{aligned}
& K_{0}=\frac{a c}{(a+c)^{2}}(\sqrt{b}+\sqrt{b-a-c})^{2}, \\
& K_{1}=\frac{a c\left[(\sqrt{b}+\sqrt{b-a-c})\left(a^{2}+a c-2 a b+2 b c\right)+\left(a^{2}-c^{2}\right) \sqrt{b}\right]}{(a+c)^{3}} .
\end{aligned}
$$

Interestingly, the numerical value of $K_{0}$ is extremely close to $b-a$ :

$$
K_{0} \equiv \lim _{\tilde{m}_{A} \rightarrow \infty} d=(212.047 \mathrm{GeV})^{2} \leftrightarrow b-a=(212.132 \mathrm{GeV})^{2}
$$

Since $K_{0}$ is the leading order prediction for $d$, (3.4) implies that even in region $(4,1)$, the relation (2.12) will still hold to a very good approximation - any deviations from it will be $1 / \tilde{m}_{A}$ suppressed. We can formalize this observation by introducing the value $m_{A}^{(b)}$ which the parameter $\tilde{m}_{A}$ takes when the mass trajectory (1.7) crosses the boundary between regions $(3,1)$ and $(4,1)$. Using the continuity of the function $d\left(a, b, c, \tilde{m}_{A}\right)$, we can write

$$
b-a=K_{0}+\frac{K_{1}}{\tilde{m}_{A}^{(b)}}+\mathcal{O}\left(\frac{1}{\left(\tilde{m}_{A}^{(b)}\right)^{2}}\right),
$$

where the left-hand side is the value of $d$ in region $(3,1)$ which is given by (A.11), while the right-hand side is the value of $d$ as predicted by the Taylor expansion (3.1) in region $(4,1)$. Eliminating $K_{0}$ from $(3.5)$, we can rewrite the expansion (3.1) in the form

$$
\begin{aligned}
d\left(a, b, c, \tilde{m}_{A}\right) & \equiv b-a+\frac{K_{1}}{\tilde{m}_{A}}-\frac{K_{1}}{m_{A}^{(b)}}+\mathcal{O}\left(\frac{1}{\tilde{m}_{A}^{2}}\right) \\
& =b-a-K_{1} \frac{\tilde{m}_{A}-m_{A}^{(b)}}{\tilde{m}_{A} m_{A}^{(b)}}+\mathcal{O}\left(\frac{1}{\tilde{m}_{A}^{2}}\right),
\end{aligned}
$$

which manifestly shows that the deviations from the relation (2.12) are $1 / m_{A}$ suppressed. One can check that the sign of the $K_{1}$ coefficient (3.3) is positive, then (3.6b) explains why $d$ is a decreasing function of $\tilde{m}_{A}$ in region $(4,1)$, as observed in the left panel of figure 7 .

Starting from (A.63b), one can repeat the same analysis for the magenta portion of the auxiliary branch which is located in region $(4,3)$. As the left panel of figure 7 shows, the conclusions will be the same - the $d$ endpoint is still given approximately by the "bad" relation (2.12), and the corrections to it are tiny and $1 / m_{A}$ suppressed. The right panel in 
figure 7 explicitly demonstrates that the variation of the $d$ endpoint along the flat direction is unnoticeable by eye even with perfect resolution, large statistics and no background. The shapes of the $m_{j \ell(\mathrm{hi})}$ distributions are also very similar. As a result, we anticipate that the additional measurement of the $d$ kinematic endpoint and the analysis of the associated $m_{j \ell(\text { hi })}$ distribution will not help much in lifting the degeneracy of the flat direction (1.7).

We now turn to the discussion of the fifth and final kinematic endpoint, $e$, illustrated in figure 8. The left panel now shows a more promising result - the variation along the flat direction is much larger than what we saw previously in figure 7 . This is especially noticeable for the auxiliary branch, where the prediction for $\sqrt{e}$ can vary by as much as $17 \mathrm{GeV}$, suggesting that one might be able to at least rule out some portions of it. At the same time, the variation of $\sqrt{e}$ along the true branch is only $4 \mathrm{GeV}$, once again making it rather difficult to pinpoint an exact location along the true branch. Unfortunately, these theoretical considerations are dwarfed by the experimental challenges in measuring the $e$ endpoint, as suggested by the right panel of figure 8. Unlike the other four kinematic endpoints, $e$ is a lower endpoint (a.k.a. "threshold"), which places it in a region where one expects more background. More importantly, the signal distribution is very poorly populated near its lower endpoint - the vast majority of signal events appear sufficiently far away from the threshold, and the measurement will suffer from a large statistical uncertainty. This casts significant doubts on the feasibility of this measurement - in previous studies, the $\sqrt{e}$ endpoint was either the measurement with the largest experimental error from the fit (on the order of $10 \mathrm{GeV}$ [79]), or one could not obtain a measurement for it at all [81]. One could hope to improve on the precision by utilizing shape information [101], but this introduces additional systematic uncertainty, since the background shape and the shape distortion due to cuts has to be modeled with Monte Carlo.

Being mindful of the challenges involved with the measurement of the $e$ endpoint, in this paper we shall look for an alternative method for lifting the degeneracy along the flat direction. Our proposal is to study the shape of the kinematic boundary (1.9), which is a two-dimensional surface in the three-dimensional space of observables

$$
\left\{m_{j \ell(\mathrm{lo})}^{2}, m_{j \ell(\mathrm{hi})}^{2}, m_{\ell \ell}^{2}\right\} \text {. }
$$

As a proof of principle, we first illustrate the change in the shape of the surface (1.9) as we move along the flat direction. Our results are shown in figure 9 (for the true branch) and in figure 10 (for the auxiliary branch). Following [93], we visualize the surface (1.9) by showing a series of two-dimensional slices in the $\left(m_{j \ell(\mathrm{lo})}^{2}, m_{j \ell(\mathrm{hi})}^{2}-m_{j \ell(\mathrm{lo})}^{2}\right)$ plane, where the slight modification of the " $y$-axis" was done in order to avoid wasted space on the plots due to the unphysical areas with $m_{j \ell(\mathrm{lo})}>m_{j \ell(\mathrm{hi})}$. Each slice is taken at a fixed value of $m_{\ell \ell}^{2}$, starting from a very low value $\left(10 \mathrm{GeV}^{2}\right)$ and going up all the way until the kinematic endpoint $\left(m_{\ell \ell}^{\max }\right)^{2}=20,976 \mathrm{GeV}^{2}$. The red solid lines in figure 9 correspond to the nominal case of the study point $P_{31}$. In each panel, the signal events will be populating the areas delineated by these red solid lines. As pointed out in [92], the density of signal events is enhanced near the phase space boundary, i.e. signal events will cluster close to the solid red lines; this property can be incorporated into the algorithm for detecting the surface boundary [93]. It is worth noting that in general, each panel contains two signal 

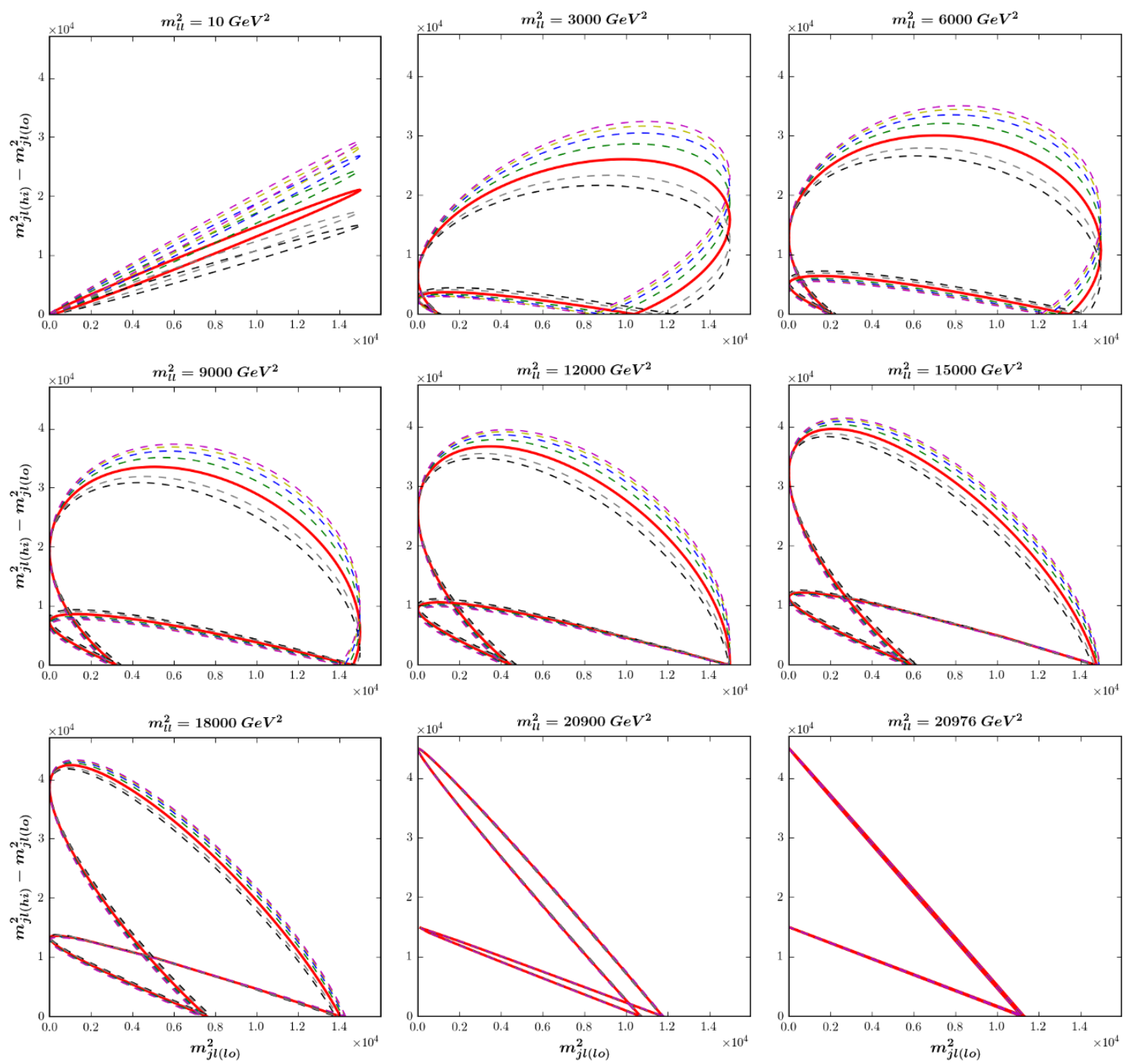

Figure 9. Signal kinematic boundaries in the $\left(m_{j \ell(\mathrm{lo})}^{2}, m_{j \ell(\mathrm{hi})}^{2}-m_{j \ell(\mathrm{lo})}^{2}\right)$ plane, at nine fixed values of $m_{\ell \ell}^{2}$. Results are shown for several points along the true branch in regions $(3,1)$ and $(4,1)$. The red solid line represents the case of the $P_{31}$ study point with $\tilde{m}_{A}=236.6 \mathrm{GeV}$, while the dashed lines correspond to other values of $\tilde{m}_{A}$ along the true branch: $\tilde{m}_{A}=0$ (black), $\tilde{m}_{A}=100 \mathrm{GeV}$ (gray), $\tilde{m}_{A}=500 \mathrm{GeV}$ (green), $\tilde{m}_{A}=1000 \mathrm{GeV}$ (blue), $\tilde{m}_{A}=2000 \mathrm{GeV}$ (yellow) and $\tilde{m}_{A}=5000 \mathrm{GeV}$ (magenta).

populations, which arise from the reordering (1.3)-(1.4) [89]. As we vary the value of $m_{\ell \ell}^{2}$, the shape of the red solid lines changes in accordance with eq. (1.9), which follows from simple phase space considerations. However, the main purpose of figures 9 and 10 is to check how much the shape is modified relative to the nominal case of $P_{31}$ when we vary the value of $\tilde{m}_{A}$ along the flat direction (1.7). The dashed lines in figure 9 show results for several representative values of $\tilde{m}_{A}$ along the true branch: $\tilde{m}_{A}=0$ (black), $\tilde{m}_{A}=100 \mathrm{GeV}$ (gray), $\tilde{m}_{A}=500 \mathrm{GeV}$ (green), $\tilde{m}_{A}=1000 \mathrm{GeV}$ (blue), $\tilde{m}_{A}=2000 \mathrm{GeV}$ (yellow) and $\tilde{m}_{A}=5000 \mathrm{GeV}$ (magenta). We observe noticeable shape variations, especially at low to intermediate values of $m_{\ell \ell}^{2}$, which bodes well for our intended purpose of measuring the 

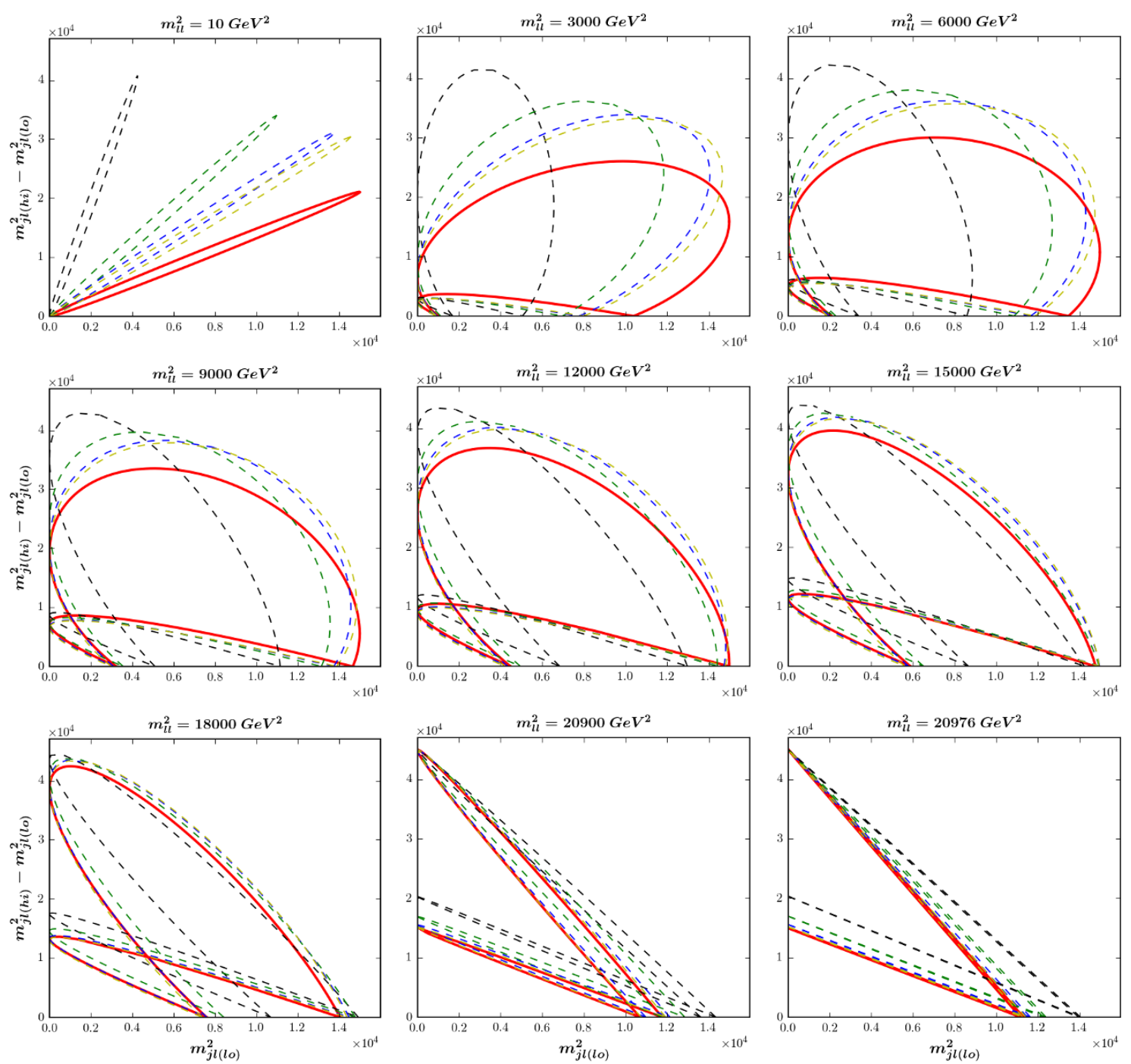

Figure 10. The same as figure 9, but for the auxiliary branch going through regions $(2,3)$ and $(4,3)$. The dashed lines represent points with $\tilde{m}_{A}=100 \mathrm{GeV}$ (black), $\tilde{m}_{A}=500 \mathrm{GeV}$ (green), $\tilde{m}_{A}=2000 \mathrm{GeV}$ (blue) and $\tilde{m}_{A}=6000 \mathrm{GeV}$ (yellow). For reference, we also show the case of the true mass spectrum for point $P_{31}$ (red solid lines), although $P_{31}$ does not belong to the auxiliary branch.

value of $m_{A}$. Figure 9 aids in visualizing why sensitivity is lost when performing onedimensional projections. Consider, for example the variable $m_{j \ell(\mathrm{lo})}$. The top two rows of figure 9 show that as $\tilde{m}_{A}$ is varied along the flat direction, the boundary contours are being stretched vertically, which does not have any effect on the $m_{j \ell(\mathrm{lo})}$ endpoint. Later on, when the events are projected vertically on the $m_{j \ell(\mathrm{lo})}$ axis to obtain the $m_{j \ell(\mathrm{lo})}$ distribution seen in the middle panel of figure 6 , the effects from this vertical stretching tend to be washed out and the resulting $m_{j \ell(10)}$ distributions have very similar shapes.

Figure 10 shows the analogous results for the auxiliary branch. Once again, the red solid lines represent the study point $P_{31}$, while the dashed lines correspond to four values of $\tilde{m}_{A}: \tilde{m}_{A}=100 \mathrm{GeV}$ (black), $\tilde{m}_{A}=500 \mathrm{GeV}$ (green), $\tilde{m}_{A}=2000 \mathrm{GeV}$ (blue) and 
$\tilde{m}_{A}=6000 \mathrm{GeV}$ (yellow). This time the shape variation along the flat direction is much more significant compared to what we saw in figure 9 . This observation agrees with our expectation based on figure 8 that points on the auxiliary branch behave quite differently from our nominal study point $P_{31}$, especially at low $\tilde{m}_{A}$.

\subsection{A toy study with uniformly distributed background}

In the remainder of this section we shall illustrate our proposed method for mass measurement with two exercises. In each case, we shall assume that the standard set of onedimensional kinematic endpoints (1.2) has already been well measured and used to reduce the relevant mass parameter space (1.1) to the flat direction (1.7) parametrized by the test mass $\tilde{m}_{A}$ for the lightest new particle $A$. This is done only for simplicity - in principle, our method would also work without any prior information from endpoint measurements, but by using those, we are reducing the 4-dimensional optimization problem in (1.17) to the much simpler one-dimensional optimization problem

$$
\max _{\tilde{m}_{A}} \bar{\Sigma}\left(\tilde{m}_{A}, \tilde{m}_{B}\left(\tilde{m}_{A}\right), \tilde{m}_{C}\left(\tilde{m}_{A}\right), \tilde{m}_{D}\left(\tilde{m}_{A}\right)\right) \simeq \bar{\Sigma}\left(m_{A}, m_{B}, m_{C}, m_{D}\right),
$$

where $\tilde{m}_{B}\left(\tilde{m}_{A}\right), \tilde{m}_{C}\left(\tilde{m}_{A}\right)$, and $\tilde{m}_{D}\left(\tilde{m}_{A}\right)$ are the masses of particles $B, C$ and $D$ along the flat direction. Our main emphasis here is on demonstrating the advantages of our method relative to the method of kinematic endpoints. In section 3.1 we already showed that while the method of kinematic endpoints does a good job in reducing the unknown mass parameter space (1.1) to the flat direction (1.7), it does a poor job of lifting the degeneracy along the flat direction. Thus, if we can show that our method can perform the remaining mass measurement along the flat direction, we will have accomplished our goal.

In order to make contact with our previous studies in [93], we begin with a simple toy exercise where in addition to the signal events from the cascade decay in figure 1, we also consider a certain number of background events, which we take to be uniformly distributed in the mass squared space of observables (3.7). While the assumption of uniform background density is unrealistic, such an exercise is nevertheless worth studying for several reasons. First, our method is completely general and applies in any situation where we have a decay of the type shown in figure 1, while to correctly identify the relevant backgrounds, we must be a lot more specific - we need to fix the signature, the type of production mechanism (which determines what else is in the event), the cuts, etc. In order to retain generality, we choose to avoid specifying those details and instead we generate background events by pure Monte Carlo according to a flat hypothesis. Second, as shown in [93], a uniform background distribution is actually a pretty good approximation to more realistic backgrounds resulting, e.g., from dilepton $t \bar{t}$ events (compare to the results in section 3.3 below). Finally, our method is attempting to detect a discontinuity in the measured event density caused by a signal kinematic boundary, so the exact shape of the background distribution is not that important, as long as it is smooth and without any sharp kinematic features. 

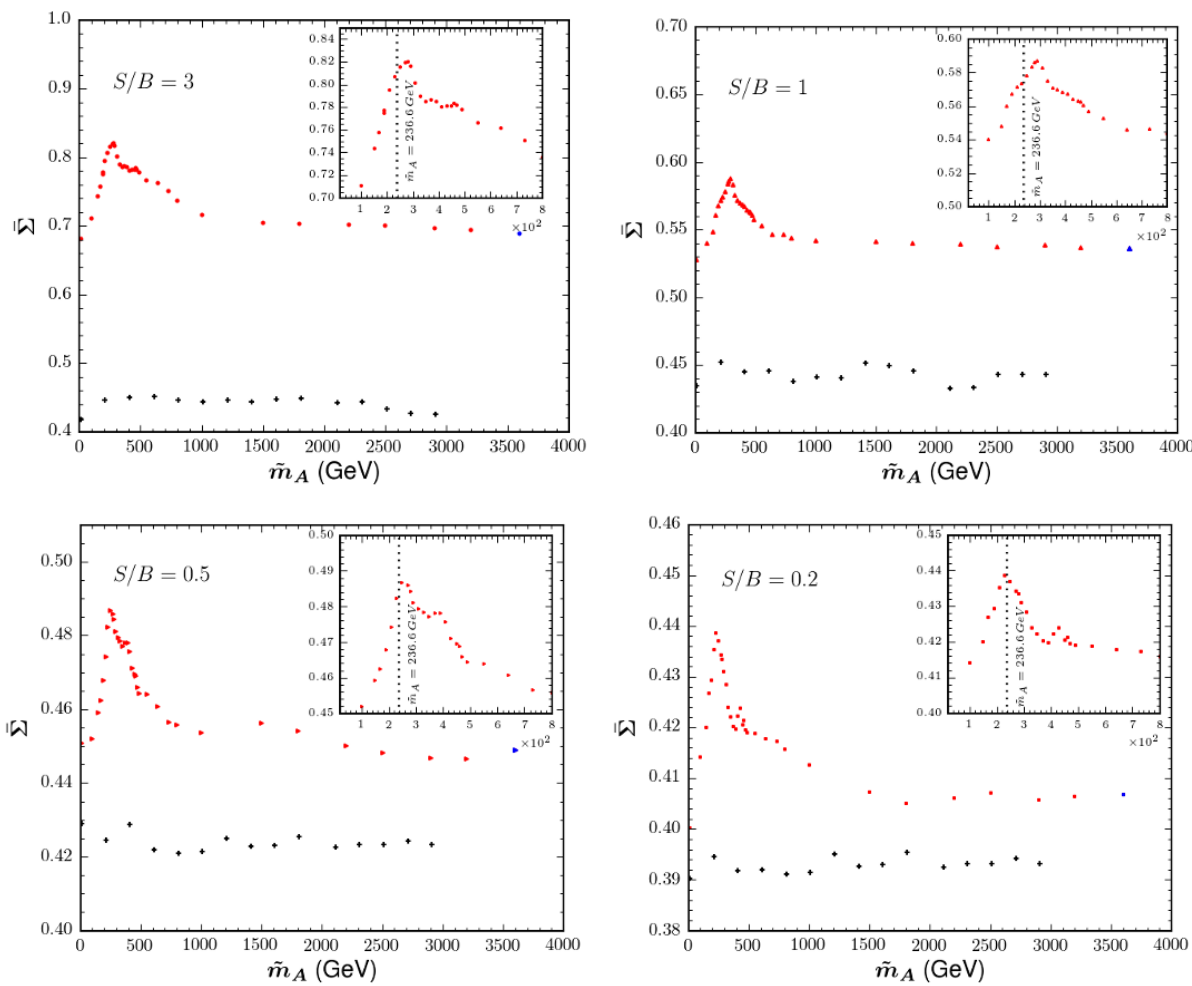

Figure 11. The quantity $\bar{\Sigma}\left(\tilde{m}_{A}\right)$ defined in (3.9) as a function of $\tilde{m}_{A}$ for different values of the signal to background ratio $S / B$ defined in (3.10): $S / B=3$ (upper left panel), $S / B=1$ (upper right panel), $S / B=0.5$ (lower left panel) and $S / B=0.2$ (lower right panel). The colored symbols correspond to the true branch with the color conventions from figure 2, while the black crosses indicate points on the auxiliary branch. The insert on each panel zooms in on the region near the peak value for $\bar{\Sigma}\left(\tilde{m}_{A}\right)$.

In order to detect the exact location of the kinematic boundary, we shall be computing the quantity $\bar{\Sigma}$ defined in (1.16) along the flat direction (1.7), i.e.

$$
\bar{\Sigma}\left(\tilde{m}_{A}\right) \equiv \bar{\Sigma}\left(\tilde{m}_{A}, \tilde{m}_{B}\left(\tilde{m}_{A}\right), \tilde{m}_{C}\left(\tilde{m}_{A}\right), \tilde{m}_{D}\left(\tilde{m}_{A}\right)\right)
$$

We shall perform several versions of the exercise, with varying levels of signal-tobackground. For this purpose, we vary the ratio of signal to background events inside the true "samosa" surface $\mathcal{S}\left(m_{A}, m_{B}, m_{C}, m_{D}\right)$ :

$$
S / B \equiv \frac{\int_{V_{\mathcal{S}}} \rho_{s} d V}{\int_{V_{\mathcal{S}}} \rho_{b} d V},
$$

where $V_{\mathcal{S}}$ is the volume inside the samosa $\mathcal{S}\left(m_{A}, m_{B}, m_{C}, m_{D}\right)$, while $\rho_{s}$ and $\rho_{b}$ are the signal and background event densities from section 1, respectively. In this exercise, we shall fix the overall normalization by choosing $N_{B}=1000$ background events inside $\mathcal{S}$.

Our main result is shown in figure 11 , which plots the quantity $\bar{\Sigma}\left(\tilde{m}_{A}\right)$ along the flat direction, for several different choices of $S / B: S / B=3$ (upper left panel), $S / B=1$ (upper right panel), $S / B=0.5$ (lower left panel) and $S / B=0.2$ (lower right panel). Each panel 
contains two sets of points: the colored symbols represent points on the true branch, while the black crosses indicate points on the auxiliary branch. ${ }^{14}$

There are several important lessons from figure 11:

- Viability of the method. We see that in each panel, the maximum of $\bar{\Sigma}$ is obtained for a value of $\tilde{m}_{A}$ which is close to the true value $m_{A}=236.6 \mathrm{GeV}$. This validates our conjecture, ${ }^{15}$ eq. (1.17), and proves the viability of our method.

- Precision of the method. Of course, we did not recover exactly the input value for $m_{A}$, but in each case, came relatively close. Each panel of figure 11 contains an insert which zooms in on the region near the peak, which is sampled more finely. For the different values of $S / B=\{3.0,1.0,0.5,0.2\}$, the $\bar{\Sigma}$ maxima are obtained at $\tilde{m}_{A}=\{280,290,250,230\} \mathrm{GeV}$, correspondingly. Since the measurement is not perfect, it may be instructive to compare the theoretical boundary for the input study point $P_{31}$ to the boundary surface found by the fit. This is illustrated in figure 12 , where in analogy to figures 9 and 10 we show two-dimensional slices at fixed $m_{\ell \ell}^{2}$ of the Voronoi tessellation of the data for the case of $S / B=3$. The Voronoi cells are color coded by their value of $\bar{\sigma}_{i}$ defined in (1.12). As in figures 9 and 10 , the red solid line in each panel is the expected signal boundary for the nominal case of point $P_{31}$. We notice that the cells with the highest values of $\bar{\sigma}_{i}$ are indeed clustered near the nominal boundary, in agreement with the results from refs. [93, 95]. On the other hand, the boundary delineated by the black dashed lines in figure 12 corresponds to the best fit value of $\tilde{m}_{A}=280 \mathrm{GeV}$, which was found in the upper left panel in figure 11. The difference between the solid red and black dashed contours in figure 12 is essentially a measure of the resolution of our method.

- Elimination of the auxiliary branch and the large $\tilde{m}_{A}$ tail of the true branch. One very positive piece of news from figure 11 is that the whole auxiliary branch has very low values for $\bar{\Sigma}$ which makes it easy to rule it out - one can see that no point on the auxiliary branch was ever in contention for the top spot. Similar comments, albeit to a lesser extent, also apply to the long tail along the true branch at large $\tilde{m}_{A}$. In particular, region $(4,1)$ seems to be ruled out, as well as the large $\tilde{m}_{A}$ portion of region $(3,1)$. In effect, the range of possible values for $\tilde{m}_{A}$ along the flat direction has been significantly narrowed down to a small interval within a few tens of $\mathrm{GeV}$ of the true value $m_{A}$.

- The adverse effect of the background. Comparing the different panels in figure 11, we see that as we make $S / B$ smaller, the difference between the true and auxiliary branch is reduced, but the auxiliary branch is still disfavored. As for the true branch, the peak near $m_{A}$ still persists, even in the case when the data is dominated by background events. This is not surprising, since the background distribution is relatively smooth, so that in the background-dominated regions of phase space there aren't too many Voronoi cells with large values of $\bar{\sigma}_{i}$, which could adversely affect the fit.

\footnotetext{
${ }^{14}$ Recall from figure 4 that for any given choice of $\tilde{m}_{A}$, there is one point on the true branch and a corresponding point on the auxiliary branch.

${ }^{15}$ Strictly speaking, figure 11 tests only the one-dimensional version (3.8).
} 

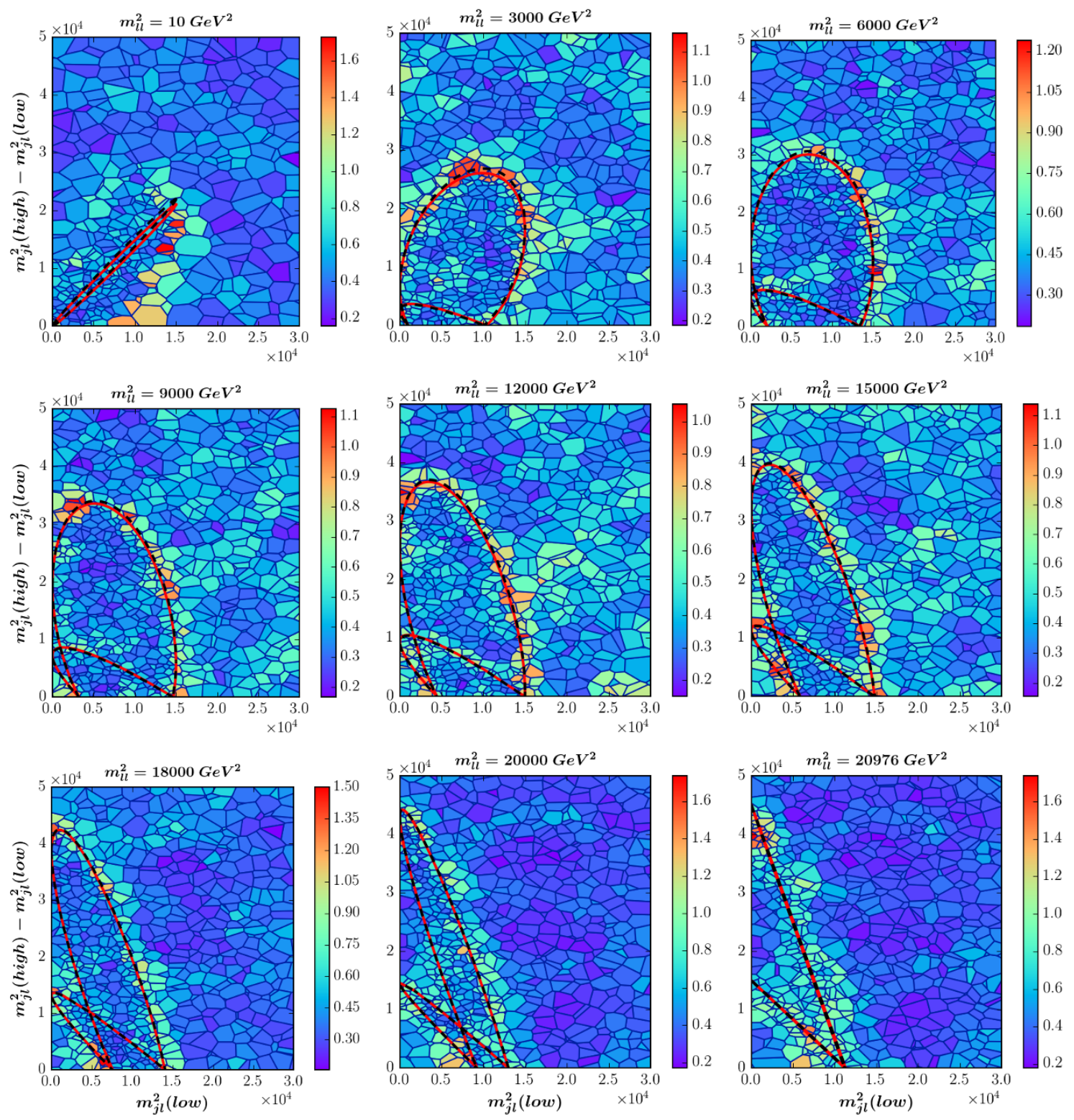

Figure 12. Two-dimensional views at fixed $m_{\ell \ell}^{2}$ of the Voronoi tessellation of the data for the case of $S / B=3$. The red solid line is the expected signal boundary for the nominal case of point $P_{31}$, i.e., with the true value $\tilde{m}_{A}=m_{A}=236.6 \mathrm{GeV}$. The black dashed line corresponds to the mass spectrum with $\tilde{m}_{A}=280 \mathrm{GeV}$, which was found to maximize the quantity $\bar{\Sigma}$ in the top left panel of figure 11.

\subsection{A study with $t \bar{t}$ dilepton background events}

We are now in position to repeat the exercise from section 3.2, with signal events from $D+A$ associated production and background taken from dilepton $t \bar{t}$ events, which represent the main background to the signature from figure 1 of a jet plus two opposite sign, same flavor leptons (the electroweak backgrounds involving leptonic $Z$ decays can be suppressed with a $Z$ mass veto). Events were generated at parton level for LHC at $14 \mathrm{TeV}$ with 

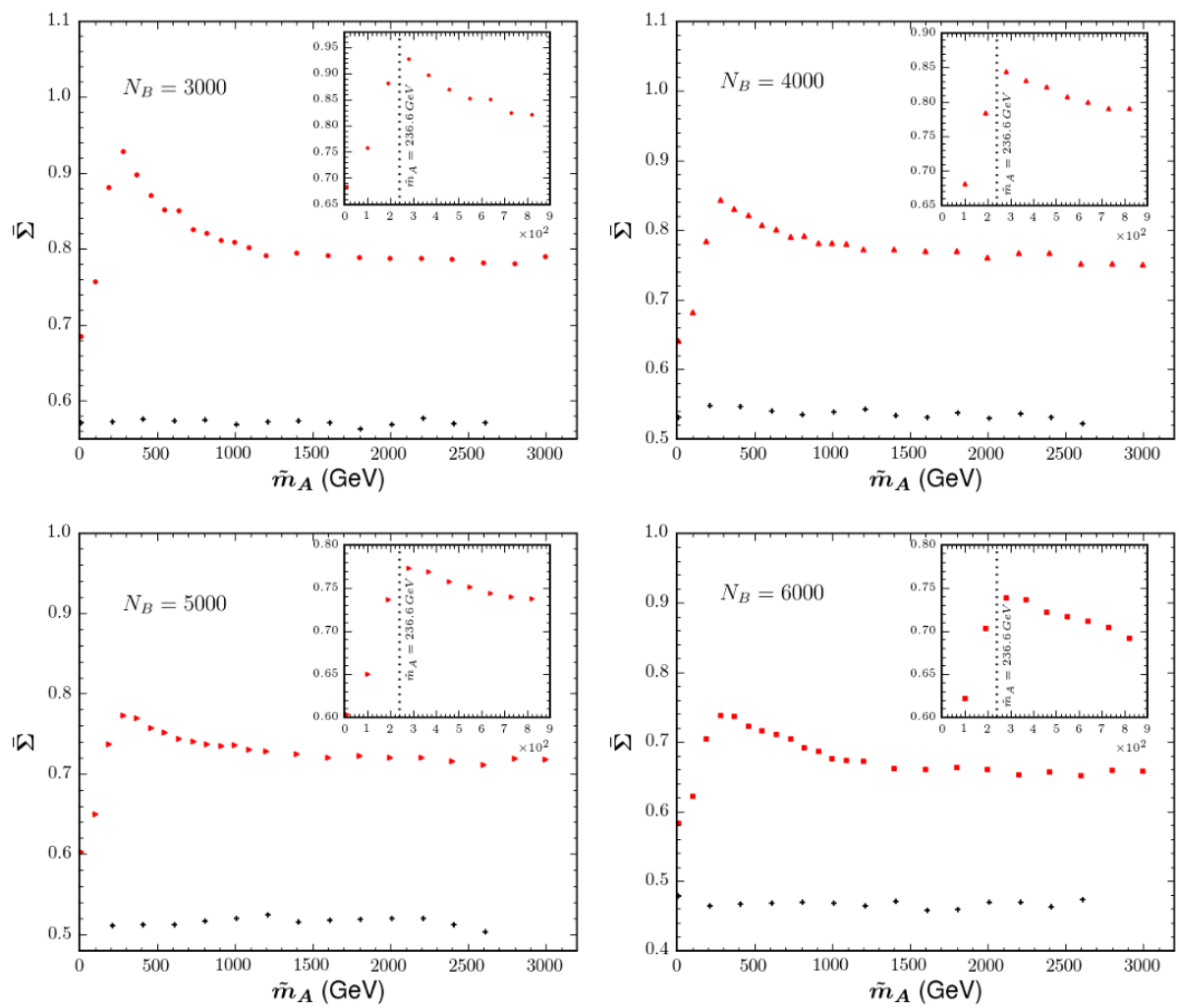

Figure 13. The analogue of figure 11 for the exercise with $t \bar{t}$ background events considered in section 3.3. Results are shown for $N_{S}=3000$ signal events and several choices for the number of background events: $N_{B}=3000$ (upper left panel), $N_{B}=4000$ (upper right panel), $N_{B}=5000$ (lower left panel) and $N_{B}=6000$ (lower right panel).

MADGraph5 [102] version 2.1.1 with the default PDF set cteq611. For signal we used the SUSY version of the cascade decay in figure 1, and considered the associate production of a squark $\tilde{q}$ with the lightest neutralino $\tilde{\chi}_{1}^{0}$, namely $p p \rightarrow \tilde{q}_{1}^{0}[103,104]$. Since each $t \bar{t}$ background event contains two jets, there is a two-fold ambiguity in the jet selection. We will use both possible pairings, so that each background event will contribute two entries to our data. Of course, we do not know a priori how many of those entries will end up inside the nominal boundary surface $\mathcal{S}\left(m_{A}, m_{B}, m_{C}, m_{D}\right)$, which is why we have to use a slightly different normalization from section 3.2. We shall fix the number of signal events to $N_{S}=3000$, and then we shall consider several values ${ }^{16}$ for the number of dilepton $t \bar{t}$ events: $N_{B}=\{3000,4000,5000,6000\}$. From Monte Carlo we then find that these choices correspond to $S / B=\{1.52,1.14,0.91,0.76\}$ inside the $\mathcal{S}$ boundary, see (3.10).

Our main result is shown in figure 13, which is the analogue of figure 11 for this case. Once again, we find that the function $\bar{\Sigma}\left(\tilde{m}_{A}\right)$ is maximized in the vicinity of $\tilde{m}_{A}=$ $m_{A}=236.6 \mathrm{GeV}$. The fitting procedure is illustrated in figure 14, which is the analogue of figure 12. The red solid lines show the boundary contours for the nominal value of

\footnotetext{
${ }^{16}$ The anticipated signal-to-background ratio is model-dependent. In this sense, SUSY may not be the best case for discovery, since other scenarios, e.g., UED [105-107], have higher signal cross-sections.
} 

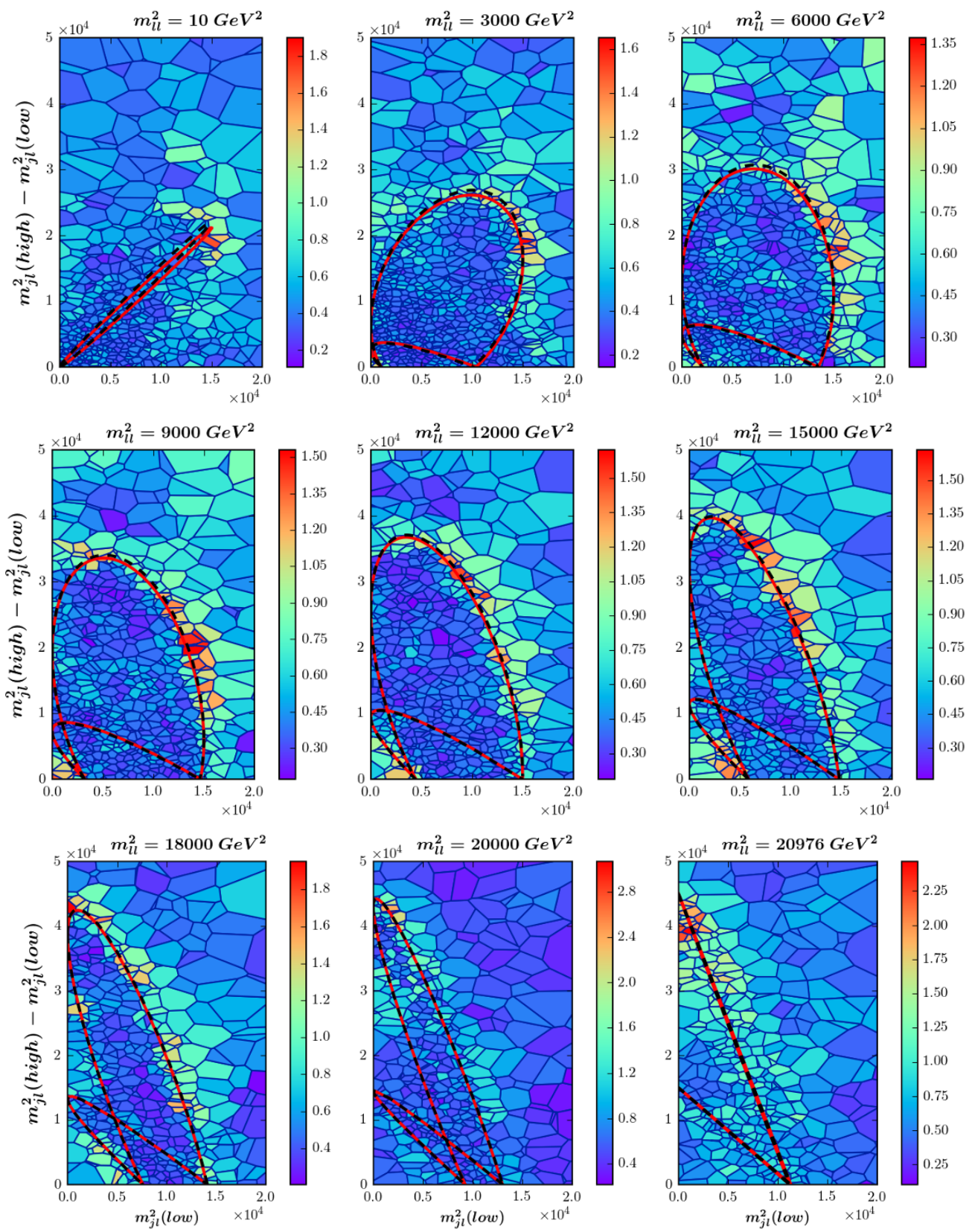

Figure 14. The analogue of figure 12 for the exercise with $t \bar{t}$ background events considered in section 3.3. The Voronoi tessellation was done for the case of $N_{B}=3000$. The red solid line is the phase space boundary for the nominal value $m_{A}=236.6 \mathrm{GeV}$, while the black dashed line corresponds to the best fit value $\tilde{m}_{A}=280.0 \mathrm{GeV}$ found in the top left panel of figure 13 .

$m_{A}=236.6 \mathrm{GeV}$, while the black dashed lines are for the best fit value of $\tilde{m}_{A}=280.0 \mathrm{GeV}$, which was found in the top left panel of figure 13. Figure 13 shows that once again, our procedure has disfavored the whole auxiliary branch and narrowed down the range of viable values of $\tilde{m}_{A}$ to a few tens of $\mathrm{GeV}$ around the nominal value $m_{A}$. 


\begin{tabular}{|c|c|c|c|c|c|c|}
\hline \multirow{2}{*}{ Region } & & \multicolumn{2}{|c|}{ true branch } & \multicolumn{3}{|c|}{ mirror branch } \\
\hline & & $(3,2)$ & $(3,1)$ & $(4,2)$ & $(4,3)$ & $(2,3)$ \\
\hline \multicolumn{2}{|l|}{ Study point } & $P_{32}$ & $P_{31}$ & $P_{42}$ & $P_{43}$ & $P_{23}$ \\
\hline \multicolumn{2}{|l|}{$m_{A}(\mathrm{GeV})$} & 126.49 & 5000.00 & 90.00 & 150.00 & 500.00 \\
\hline \multicolumn{2}{|l|}{$m_{B}(\mathrm{GeV})$} & 282.84 & 5207.42 & 194.61 & 250.96 & 609.04 \\
\hline \multicolumn{2}{|l|}{$m_{C}(\mathrm{GeV})$} & 447.21 & 5324.17 & 399.99 & 460.80 & 815.72 \\
\hline \multicolumn{2}{|l|}{$m_{D}(\mathrm{GeV})$} & 500.00 & 5372.07 & 458.78 & 518.78 & 869.36 \\
\hline \multicolumn{2}{|l|}{$R_{A B}$} & 0.200 & 0.922 & 0.214 & 0.357 & 0.674 \\
\hline \multicolumn{2}{|l|}{$R_{B C}$} & 0.400 & 0.957 & 0.237 & 0.297 & 0.557 \\
\hline \multicolumn{2}{|l|}{$R_{C D}$} & 0.800 & 0.982 & 0.760 & 0.789 & 0.880 \\
\hline$m_{l l}^{\max }(\mathrm{GeV})$ & $\sqrt{a}$ & & & 309.84 & & \\
\hline$m_{j l l}^{\max }(\mathrm{GeV})$ & $\sqrt{b}$ & & & 368.78 & & \\
\hline$m_{j l(\mathrm{lo})}^{\max }(\mathrm{GeV})$ & $\sqrt{c}$ & & & 149.07 & & \\
\hline$m_{j l(\mathrm{hi})}^{\max }(\mathrm{GeV})$ & $\sqrt{d}$ & 200.00 & 200.00 & 198.23 & 199.87 & 200.00 \\
\hline$m_{j l l\left(\theta>\frac{\pi}{2}\right)}^{\min }(\mathrm{GeV})$ & $\sqrt{e}$ & 247.94 & 237.47 & 253.72 & 250.99 & 243.81 \\
\hline
\end{tabular}

Table 2. The same as table 1, except now the starting point is a point $\left(P_{32}\right)$ from region $(3,2)$.
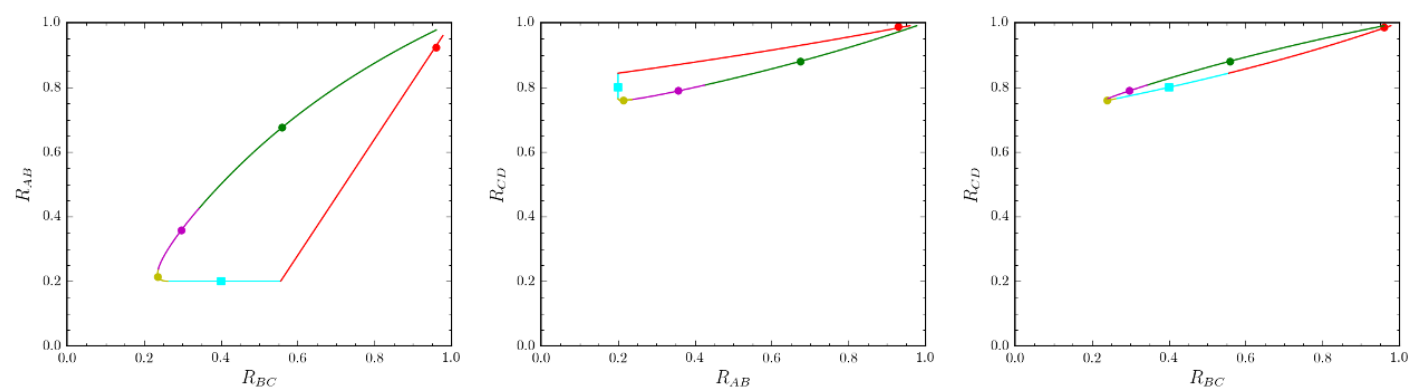

Figure 15. The same as figure 3 , but for the flat direction generated by point $P_{32}$ from table 2 .

\section{A case study in region $(3,2)$}

In this section, we shall repeat the analysis from section 3, only this time our nominal study point, from now on labelled as $P_{32}$, will be chosen within the cyan region $(3,2)$ of figure 2. Recall that the problematic relation (2.12) was satisfied in three of the colored regions in figure 2 , namely $(2,3),(3,1)$ and $(3,2)$. The former two regions, $(2,3)$ and $(3,1)$, were already visited by the mass trajectory studied in section 3 , thus here for completeness we will also illustrate the case of region $(3,2)$.

\subsection{Kinematical properties along the flat direction}

The mass spectrum for the study point $P_{32}$ and the corresponding mass squared ratios and kinematic endpoints are shown in the cyan-shaded column of table 2. Point $P_{32}$ was used 


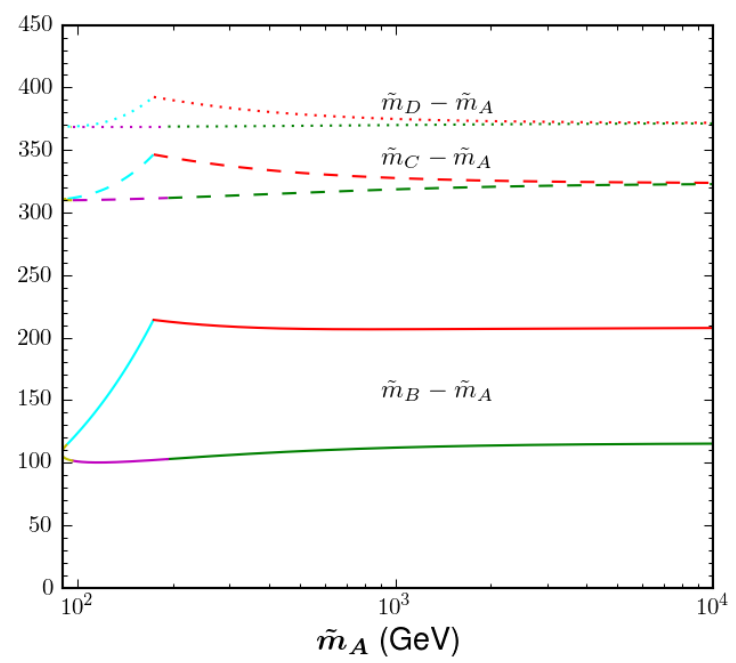

Figure 16. The analogue of figure 4 , but for the flat direction defined in figure 15 .

previously in ref. [89] as an example of a discrete two-fold ambiguity, while here it serves to define a flat direction (1.7) in mass parameter space. This flat direction is illustrated in figures 15 and 16, which are the analogues of figures 3 and 4, respectively. According to figures 15 and 16, the mass trajectory now goes through five of the six colored regions in figure 2: there is a true branch through the red region $(3,1)$, the cyan region $(3,2)$ and the yellow region $(4,2)$, as well as an auxiliary branch through the yellow region $(4,2)$, the magenta region $(4,3)$ and the green region $(2,3)$. As in section 3 , we choose one representative study point in each of these regions. The four additional study points, $P_{31}$, $P_{42}, P_{43}$ and $P_{23}$, are also listed in table 2 , and their columns are shaded with the color of their respective regions in figure 2 .

The flat direction depicted in figure 15 is again parametrized by the trial value $\tilde{m}_{A}$ for the mass of the lightest new particle $A$. However, as seen in figure 16, this time the allowed range for $\tilde{m}_{A}$ does not extend all the way to $\tilde{m}_{A}=0$, and instead the true and auxiliary branch meet inside the yellow region $(4,2)$ around at the lowest value $\tilde{m}_{A} \sim 89 \mathrm{GeV}$.

The transitions between two neighboring regions along the flat direction can be understood from figure 17, which plots the mass squared ratios $R_{A B}, R_{B C}$ and $R_{C D}$ (solid lines) and several other quantities (dotted lines) which are relevant for defining the regions from figure 2, as a function of the mass trajectory parameter $\tilde{m}_{A}$. For example, relations (A.3) and (A.13) imply that the boundary between the cyan region $(3,2)$ and the red region $(3,1)$ is given by $R_{B C}=\left(2-R_{A B}\right)^{-1}$. Indeed, the lines in figures 16 and 17 change color from cyan to red when the $R_{B C}$ curve crosses the dotted line representing the function $\left(2-R_{A B}\right)^{-1}$ near $\tilde{m}_{A}=173 \mathrm{GeV}$. Similarly, it follows from (A.21) and (A.54) that the boundary between the green region $(2,3)$ and the magenta region $(4,3)$ is given by $R_{B C}=R_{A B} R_{C D}$. The right panel of figure 17 confirms that the line color changes from magenta to green when the solid line for $R_{B C}$ is intersected by the dotted line for $R_{A B} R_{C D}$. Finally, according to (A.42) and (A.53), the transition between the yellow region $(4,2)$ and the magenta region $(4,3)$ occurs at $R_{A B}=R_{B C}$, and this is borne out by figure 17 as well. 

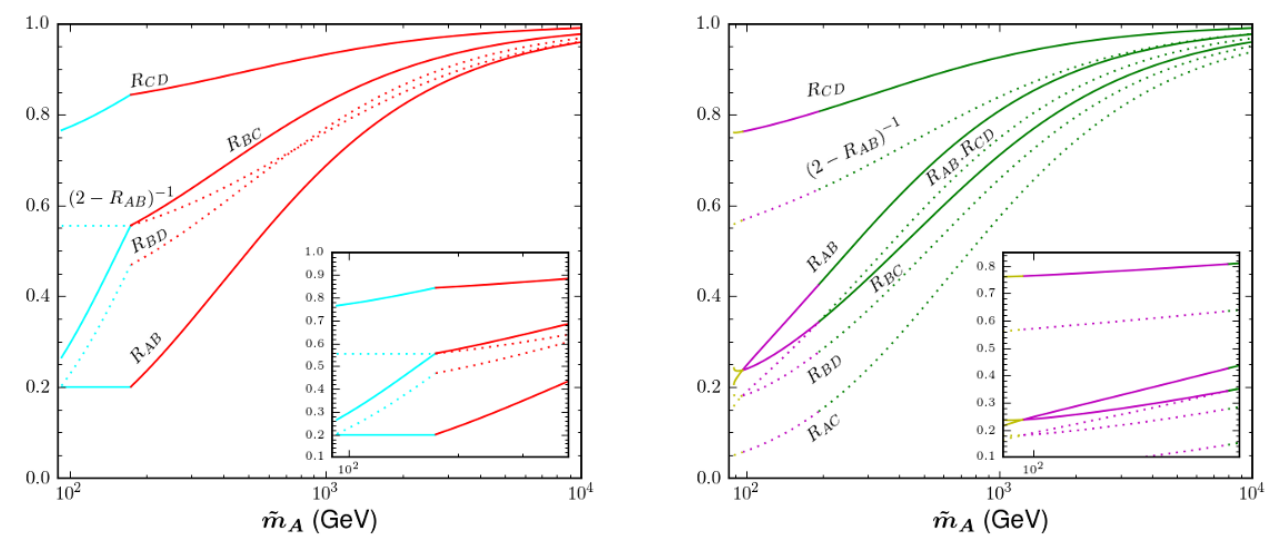

Figure 17. The equivalent representation of figure 16 in terms of the mass squared ratios $R_{A B}$, $R_{B C}$ and $R_{C D}$ (solid lines). The dotted lines depict various quantities of interest which are used to delineate the regions in figure 2. The left panel shows the true branch passing through regions $(3,2)$ (cyan) and $(3,1)(\mathrm{red})$, while the right panel shows the auxiliary branch through regions $(4,2)$ (yellow), $(4,3)$ (magenta) and $(2,3)$ (green). The left insert zooms in on the transition between regions $(3,2)$ and $(3,1)$ near $\tilde{m}_{A}=173 \mathrm{GeV}$, while the right insert focuses on the transitions between regions $(4,2)$ and $(4,3)$ near $\tilde{m}_{A}=97 \mathrm{GeV}$ and between regions $(4,3)$ and $(2,3)$ near $\tilde{m}_{A}=193 \mathrm{GeV}$.
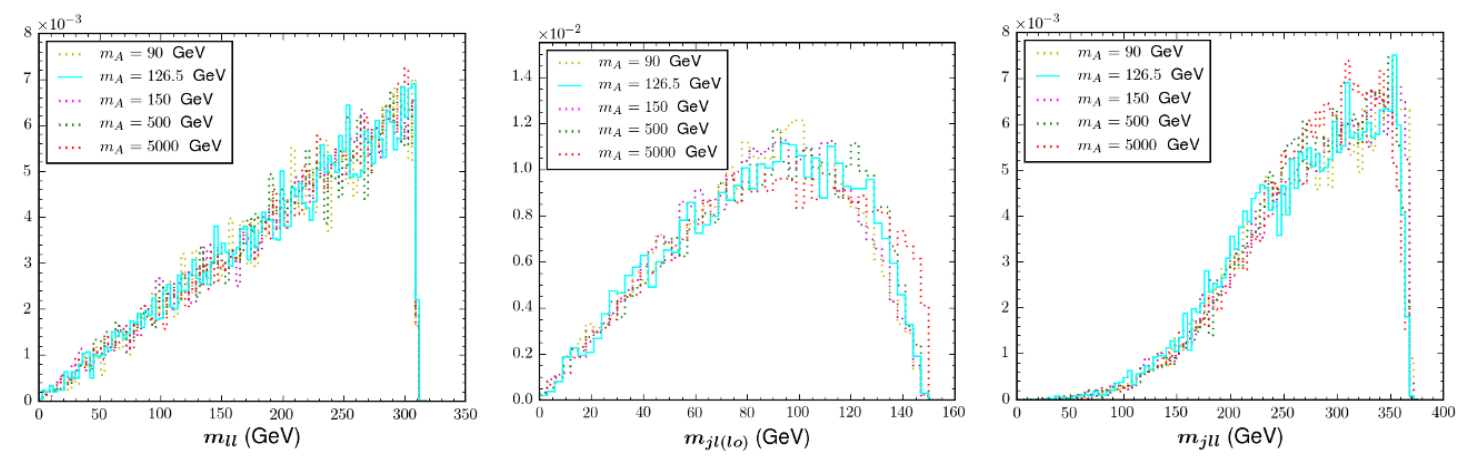

Figure 18. The analogue of figure 6 , but for the five study points exhibited in table 2. The distributions are color-coded according to our color conventions for the regions in figure 2 .

By construction, all five study points from table 2 predict identical values for the three kinematic endpoints $a, b$ and $c$. This is demonstrated in figure 18, which is the analogue of figure 6 , but for the five study points from table 2. As before, the distributions in figure 18 are color-coded according to our color conventions from figure 2 . The nominal input study point $P_{32}$ is represented by the solid line, while the dotted lines mark the other four study points. Given that the five study points look very similar on figure 18, we now focus on the remaining two distributions, $m_{j \ell(\text { hi })}$ and $m_{j l l\left(\theta>\frac{\pi}{2}\right)}$, which are investigated in figures 19 and 20. The left panels show the predictions for the kinematic endpoint $\sqrt{d}=m_{j \ell(\mathrm{hi})}^{\max }$ and the threshold $\sqrt{e}=m_{j \ell \ell\left(\theta>\frac{\pi}{2}\right)}^{\min }$, respectively, while the right panels plot the corresponding kinematic distributions for the five study points from table 2 .

By now, we should not be surprised by the extreme flatness of the curves exhibited in the left panel of figure 19. The mass trajectory from figure 15 passes through all three 

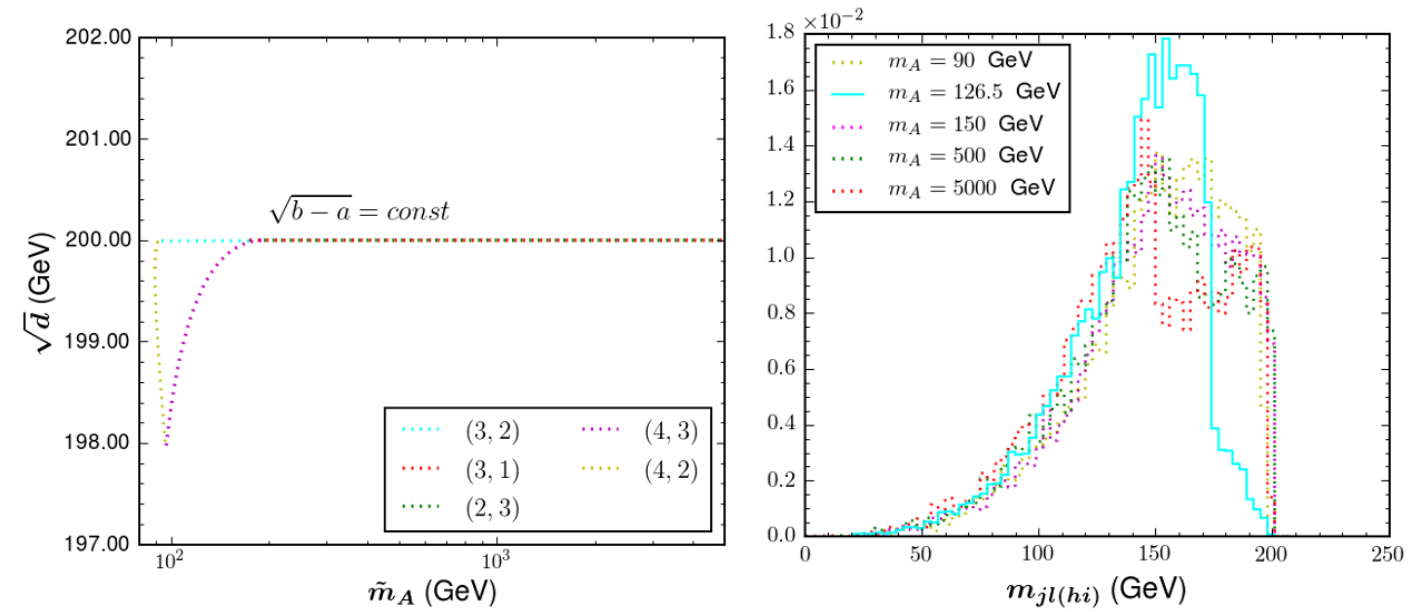

Figure 19. The analogue of figure 19, but for the flat direction defined in figure 15 (left panel) and for the five study points from table 2 (right panel).
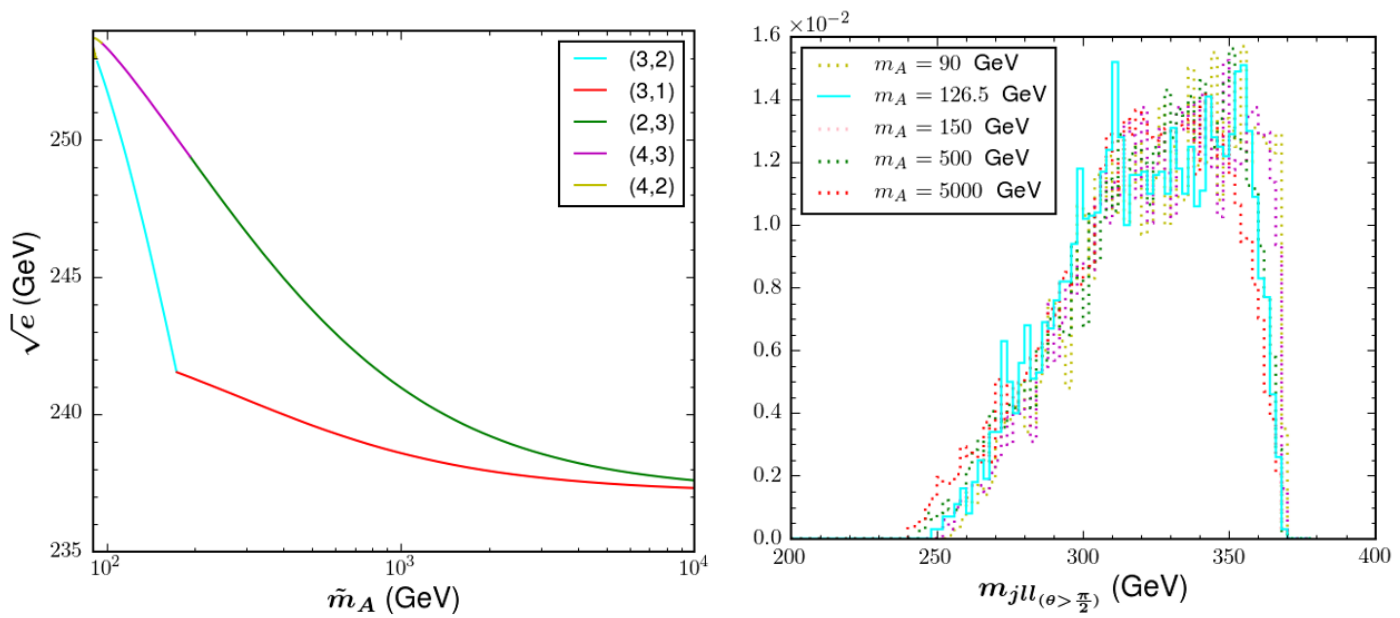

Figure 20. The same as figure 19, but for the kinematic endpoint $\sqrt{e}$ and the corresponding $m_{j l l\left(\theta>\frac{\pi}{2}\right)}$ distribution.

of the regions where the endpoint $d$ is not an independent quantity, but is fixed by the relation (2.12) and is therefore strictly independent of $\tilde{m}_{A}$. In the remaining two regions, $(4,2)$ and $(4,3)$, figure 19 shows a maximal deviation of only $2 \mathrm{GeV}$ from the prediction $\sqrt{d}=\sqrt{b-a}$ of (2.12). Taken together, the left panels of figures 7 and 19 justify our terminology of the mass trajectory (1.7) as a "flat direction" in mass parameter space.

On the other hand, the left panel of figure 20 shows a much more significant variation of the kinematic threshold variable $\sqrt{e}$ along the flat direction. The total variation is on the order of $15 \mathrm{GeV}$, which is of the same order as our previous result in figure 8 . However, as we already discussed in section 3 , the measurement of $\sqrt{e}$ presents significant experimental challenges, as one can deduce from the very minor apparent variation of the $m_{j \ell \ell\left(\theta>\frac{\pi}{2}\right)}$ distributions shown in the right panel of figure 20. This motivates searching for alternative methods for lifting the degeneracy along the flat direction. 

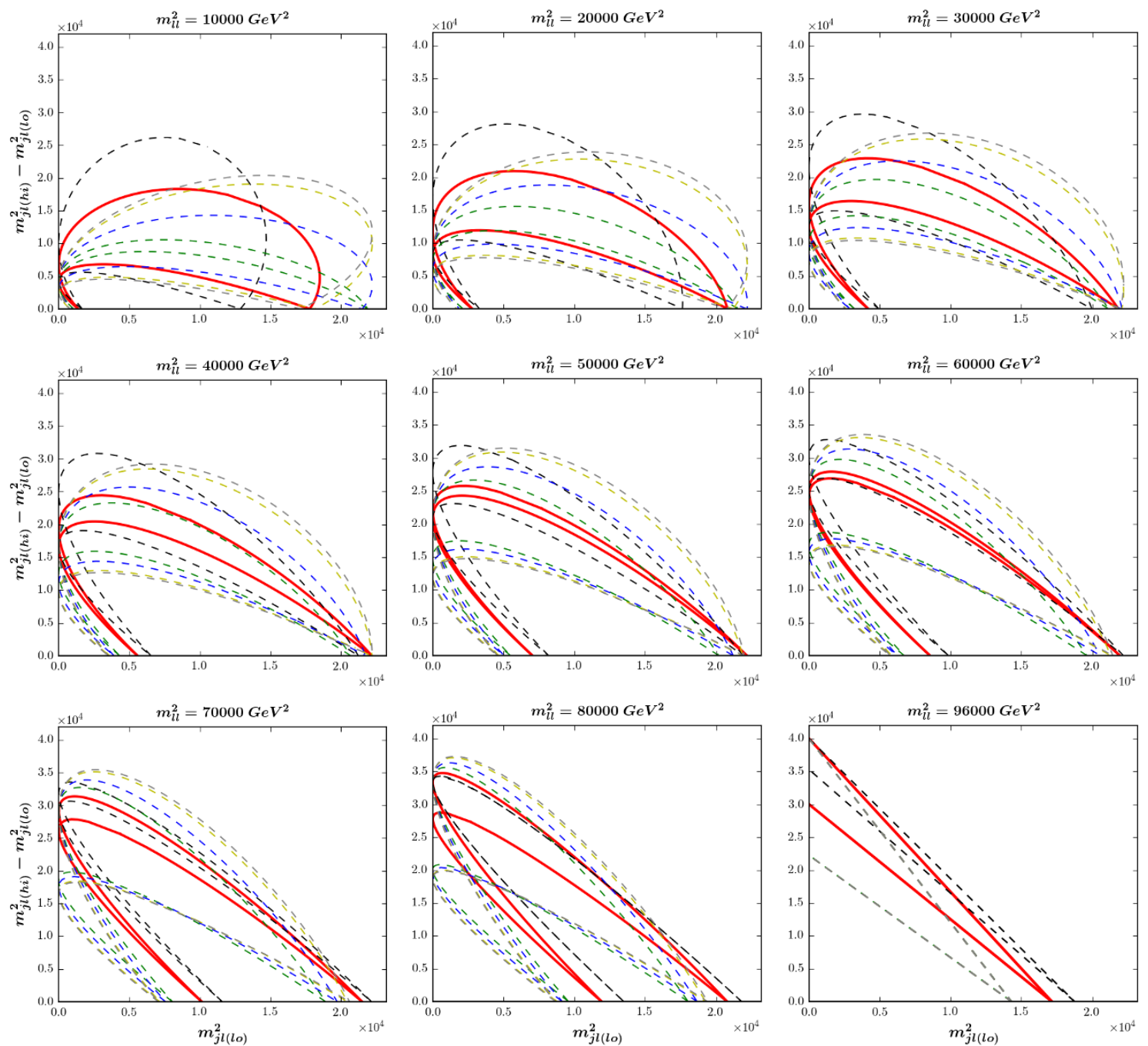

Figure 21. The same as figure 9, but for the true branch in figure 15. The red solid line represents the case of the $P_{32}$ study point with $\tilde{m}_{A}=126.5 \mathrm{GeV}$, while the dashed lines correspond to other values of $\tilde{m}_{A}$ along the true branch: $\tilde{m}_{A}=100$ (black), $\tilde{m}_{A}=173 \mathrm{GeV}$ (green), $\tilde{m}_{A}=500 \mathrm{GeV}$ (blue), $\tilde{m}_{A}=2000 \mathrm{GeV}$ (yellow) and $\tilde{m}_{A}=4000 \mathrm{GeV}$ (gray).

As already discussed in section 3 , one such method is to track the deformation of the shape of the kinematic boundary (1.9) along the flat direction. The effect is illustrated in figures 21 and 22, which are the analogues of figures 9 and 10 for the example of a flat direction considered in this section. Once again, the solid red lines in each panel indicate the kinematic boundaries for the nominal study point $P_{32}$ with $\tilde{m}_{A}=126.5 \mathrm{GeV}$, while the dashed lines are drawn for several other values of $\tilde{m}_{A}$, chosen so that they illustrate the typical range of shape fluctuations. Along the true branch, in figure 21 we plot contours for $\tilde{m}_{A}=100$ (black), $\tilde{m}_{A}=173 \mathrm{GeV}$ (green), $\tilde{m}_{A}=500 \mathrm{GeV}$ (blue), $\tilde{m}_{A}=2000 \mathrm{GeV}$ (yellow) and $\tilde{m}_{A}=4000 \mathrm{GeV}$ (gray). Even though we are confined to the true branch only, when we compare figure 21 to its analogue, figure 9 , we observe a much larger variation in the shape of the kinematic boundary in the present case, which promises good prospects for the mass 

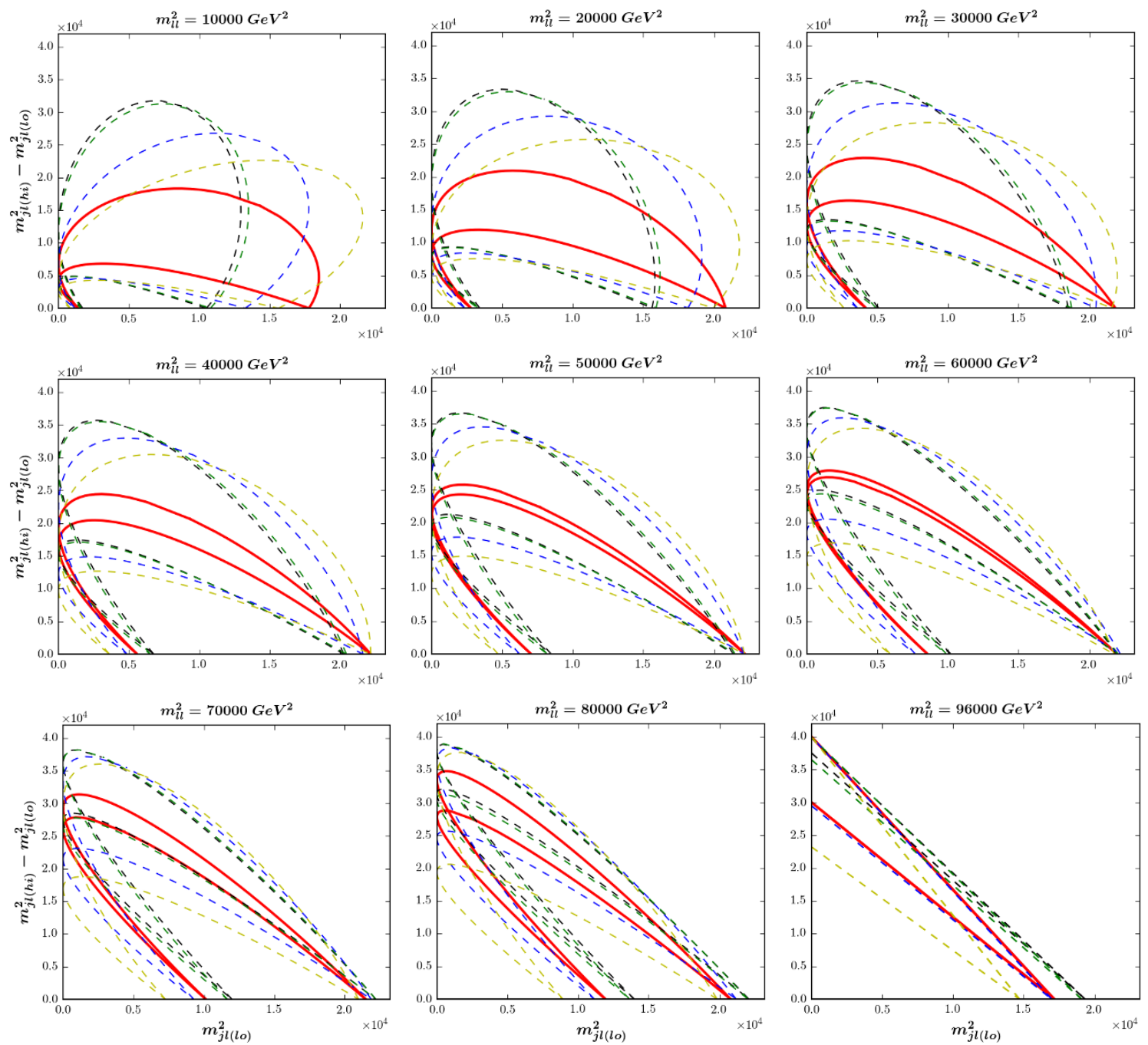

Figure 22. The same as figure 21, but for the auxiliary branch in regions $(4,2),(4,3)$ and $(2,3)$. The dashed lines represent points with $\tilde{m}_{A}=90 \mathrm{GeV}$ (black), $\tilde{m}_{A}=150 \mathrm{GeV}$ (green), $\tilde{m}_{A}=500 \mathrm{GeV}$ (blue) and $\tilde{m}_{A}=5000 \mathrm{GeV}$ (yellow). For reference, we also show the case of the true mass spectrum for point $P_{32}$ (red solid lines), although $P_{32}$ itself does not belong to the auxiliary branch.

measurement exercise to follow. The results shown in figure 22 for the auxiliary branch are also quite good. This should not come as a surprise, since the exercise in section 3 already indicated that the auxiliary branch has a different kinematic behavior, as reflected in the shape of the phase space boundary.

\subsection{A toy study with uniformly distributed background}

In the remainder of section 4 we shall repeat the two exercises from sections 4.2 and 4.3 , only this time we shall use $P_{32}$ as our input study point, and perform the measurement along the corresponding flat direction described in figures 15 and 16.

First we consider the case of uniformly distributed (in mass squared) background events, and proceed to evaluate the quantity $\bar{\Sigma}$ along the flat direction. As before, we fix 

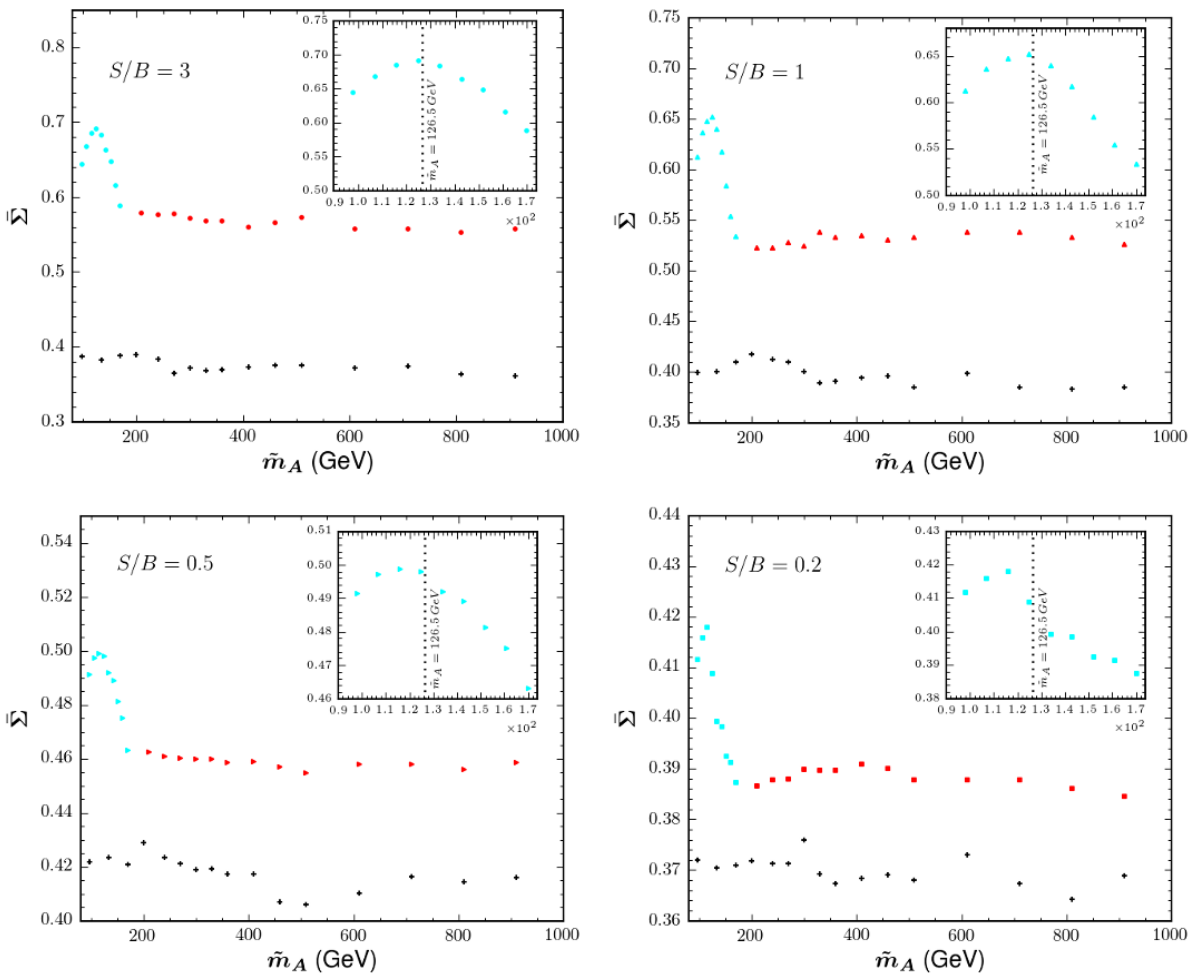

Figure 23. The same as figure 11, but now taking point $P_{32}$ as input and measuring along the flat direction depicted in figure 15 .

$N_{B}=1000$ and then vary the signal-to-background ratio inside the boundary surface $\mathcal{S}$. Figure 23 shows our results for the same choices of $S / B$ as in figure 11: $S / B=3$ (upper left panel), $S / B=1$ (upper right panel), $S / B=0.5$ (lower left panel) and $S / B=0.2$ (lower right panel). We find that the function $\bar{\Sigma}\left(\tilde{m}_{A}\right)$ once again peaks in the vicinity of the true value $m_{A}=126.5 \mathrm{GeV}$. Specifically, for $S / B=\{3.0,1.0,0.5,0.2\}$, the maxima are found at $\tilde{m}_{A}=\{125,125,116,116\} \mathrm{GeV}$, to be contrasted with the true value of $m_{A}=126.5 \mathrm{GeV}$. In all four cases, the auxiliary branch is disfavored, as it always gives low values for $\bar{\Sigma}$, while the true branch is restricted to a very narrow region near the true mass spectrum.

Figure 24 provides a consistency check on our fitting procedure, similarly to figure 12. We show two-dimensional views at fixed $m_{\ell \ell}^{2}$ of the Voronoi tessellation of the data for the case of $S / B=3$. The red solid line is the expected signal boundary for the nominal case of point $P_{32}$, i.e., with the true value $\tilde{m}_{A}=m_{A}=126.5 \mathrm{GeV}$. The black dashed line then corresponds to the best fit, i.e., a mass spectrum with $\tilde{m}_{A}=126 \mathrm{GeV}$, which was found to maximize the quantity $\bar{\Sigma}$ in the top left panel of figure 23 .

\subsection{A study with $t \bar{t}$ dilepton background events}

Our final task will be to repeat the $P_{32}$ exercise with dilepton $t \bar{t}$ events as was done in section 3.3. As before, we fix the number of signal events $N_{S}=3000$ and then consider several values for the number of background events: $N_{B}=\{3000,4000,5000,6000\}$. In each case, we compute the function $\bar{\Sigma}\left(\tilde{m}_{A}\right)$ along the flat direction of figure 15 . The results are 

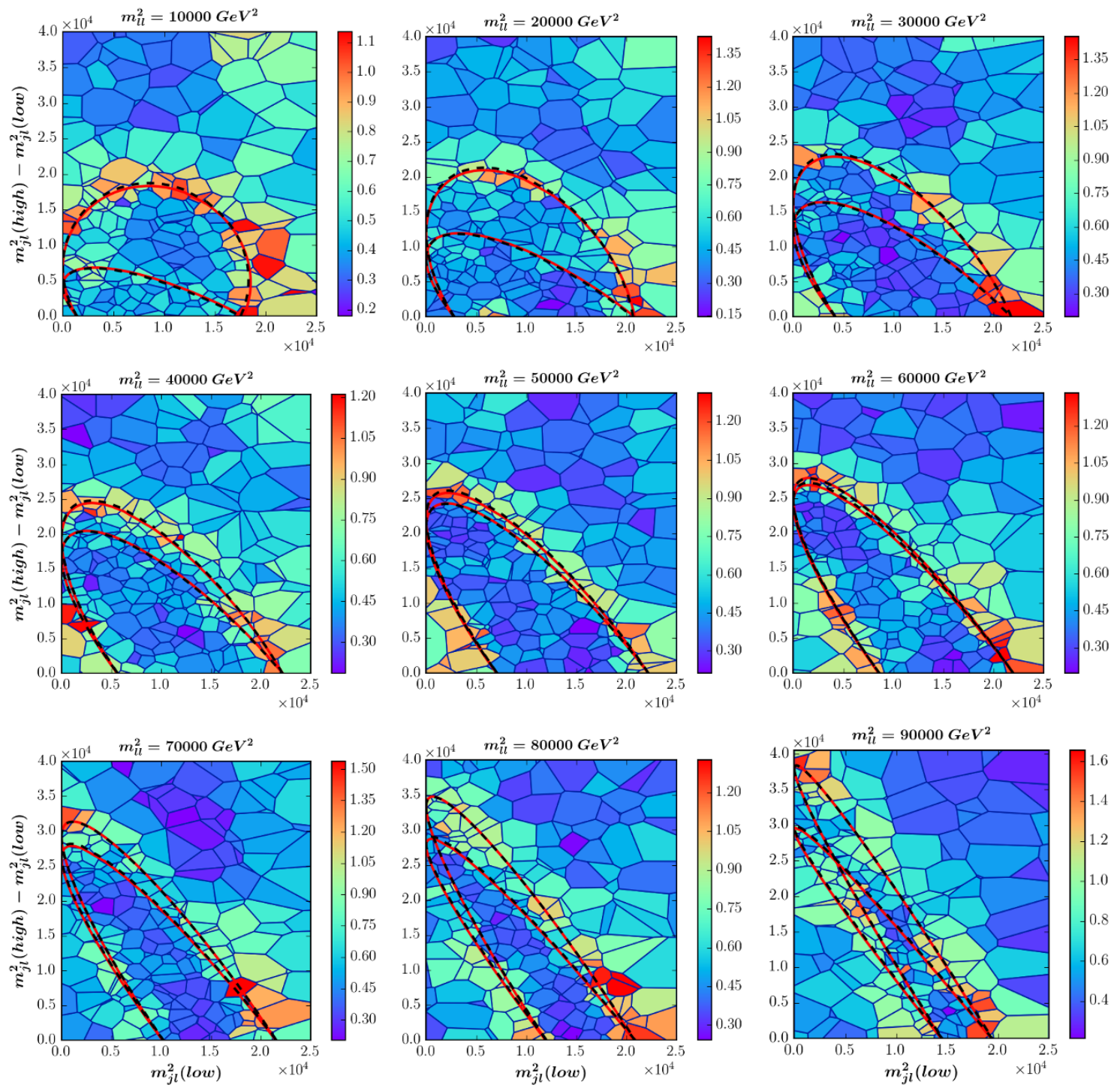

Figure 24. The same as figure 12, but for the exercise performed in section 4.2 , with point $P_{32}$ as input (solid red lines). The black dashed line corresponds to the mass spectrum with $\tilde{m}_{A}=125 \mathrm{GeV}$, which was found to maximize the quantity $\bar{\Sigma}$ in the top left panel of figure 23 .

shown in figure 25, which has the same qualitative behavior as figure 23. The $\bar{\Sigma}$ values for the auxiliary branch tend to be low, and the branch is disfavored. The global peak of $\bar{\Sigma}\left(\tilde{m}_{A}\right)$ is again found in the vicinity of the right answer (for $N_{B}=\{3000,4000,5000,6000\}$, the peak is at $\left.\tilde{m}_{A}=\{116,125,125,125\} \mathrm{GeV}\right)$, and the large $\tilde{m}_{A}$ tail of the true branch is also disfavored. One final consistency check is provided by figure 26 , which shows a comparison of the kinematic boundaries for the nominal study point $P_{32}$ with $m_{A}=126.5 \mathrm{GeV}$ (red solid lines), and the boundaries for the best fit value $\tilde{m}_{A}=125 \mathrm{GeV}$ (black dashed lines). 

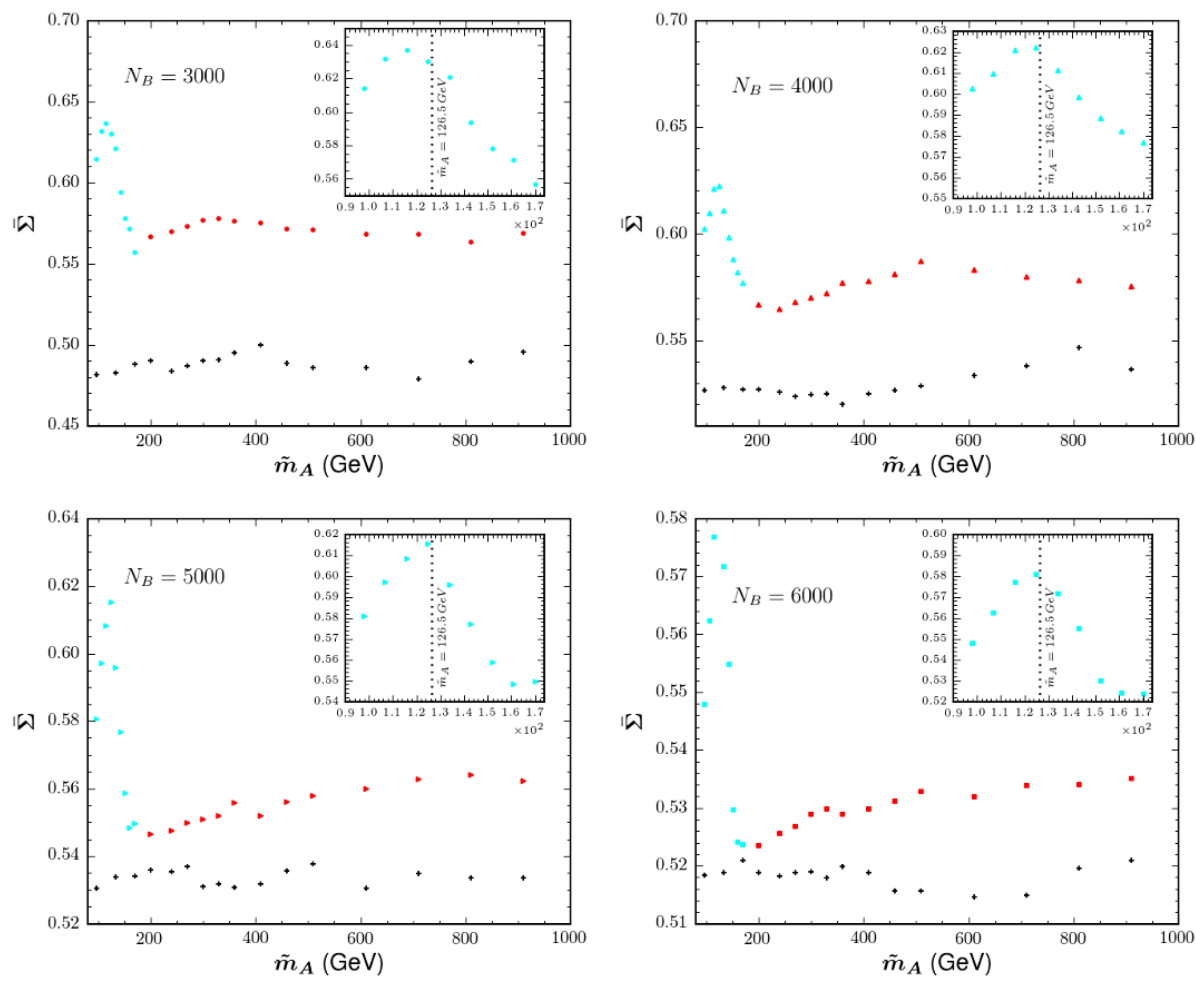

Figure 25. The same as figure 13, but using study point $P_{32}$ as input.

\subsection{A detector level study}

In this paper, we introduced the new Voronoi-based method for mass measurement as a proof of principle, and showed that at the parton level it does reasonably well in the two examples considered so far in sections 3 and 4 . Before concluding, we would like to also test the method in the presence of detector effects (this subsection) and combinatorics (see section 4.5 below). For this purpose, we first repeat the exercise from section 4.3, only this time we account for the finite detector resolution by smearing the jet energies with the typical hadronic calorimeter resolution

$$
\frac{\sigma}{E}=\left(\frac{1}{\sqrt{E}}\right)
$$

and electromagnetic calorimeter resolution

$$
\left(\frac{\sigma}{E}\right)^{2}=\left(\frac{0.0363}{\sqrt{E}}\right)^{2}+\left(\frac{0.124}{E}\right)^{2}+0.0026^{2}
$$

in CMS [108], with the energy measured in GeV. Smearing of the muon momenta is done according to the "Full System" values in figure 1.5 of [108]. The result of the fitting exercise is shown in figure 27. We see that the peak structure in the vicinity of the correct mass value $(126.5 \mathrm{GeV})$ is preserved, but somewhat degraded due to the detector resolution effects. 

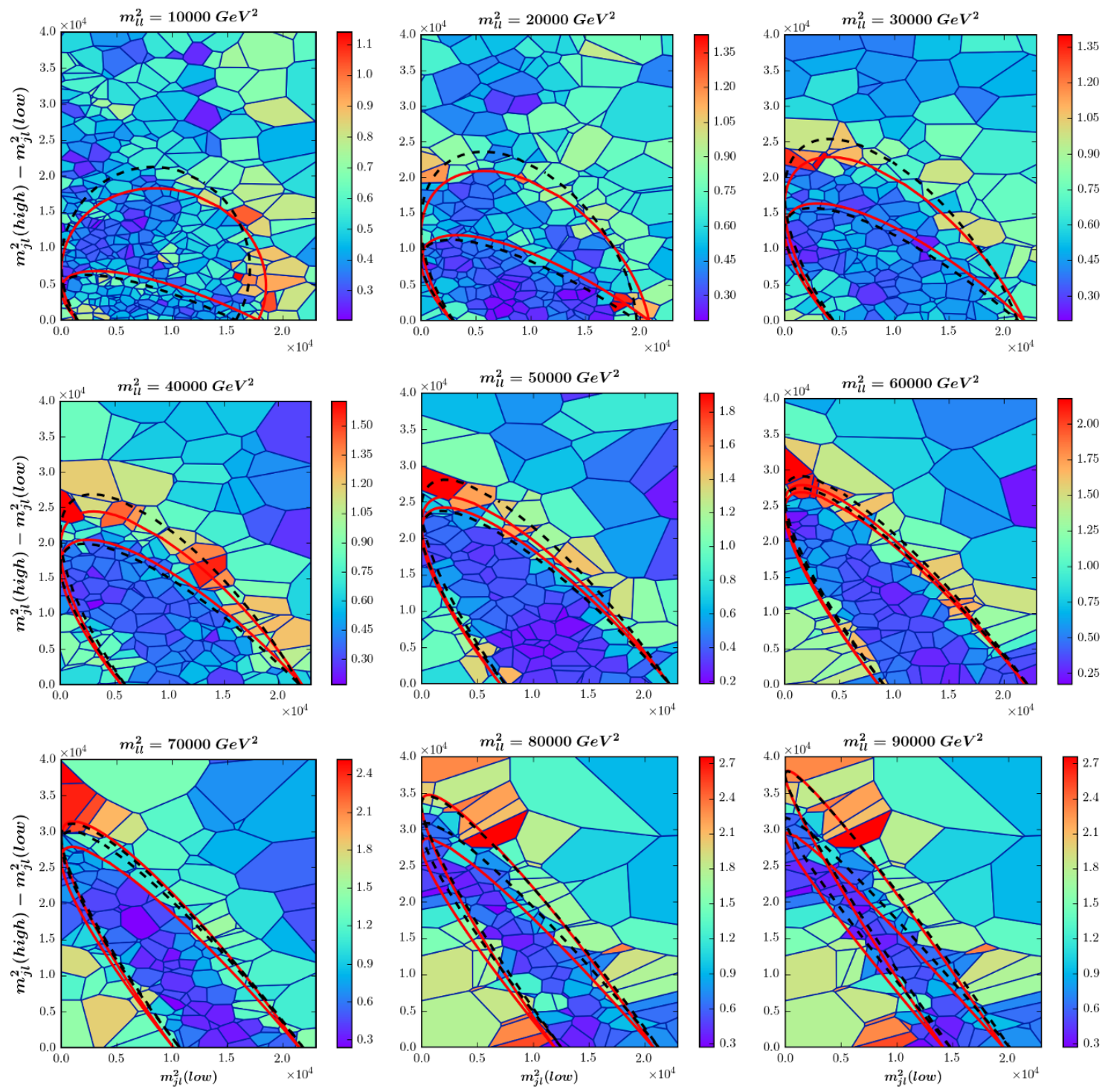

Figure 26. The same as figure 14, but for the exercise performed in section 4.3, using study point $P_{32}$ as input. The red solid line is the phase space boundary for the nominal value $m_{A}=126.5 \mathrm{GeV}$, while the black dashed line corresponds to the best fit value $\tilde{m}_{A}=116 \mathrm{GeV}$ found in the top left panel of figure 25.

\subsection{D-pair production and combinatorics effects}

Our proposed mass measurement method uses a single decay chain like the one depicted in figure 1. In that sense, the method is inclusive and model-independent, since it does not depend on what else is going on in the event. In particular, the method is equally applicable when particle $D$ is produced singly, in pairs, or in association with another object. Nevertheless, a well-motivated and widely studied class of models are the SUSYlike dark matter scenarios in which all particles $A, B, C$ and $D$ carry negative parity under the additional $Z_{2}$ symmetry. In that case, $D$ has to be produced in association with another negative parity object. If $A$ is a neutral dark matter candidate, then $D$ must carry color, 

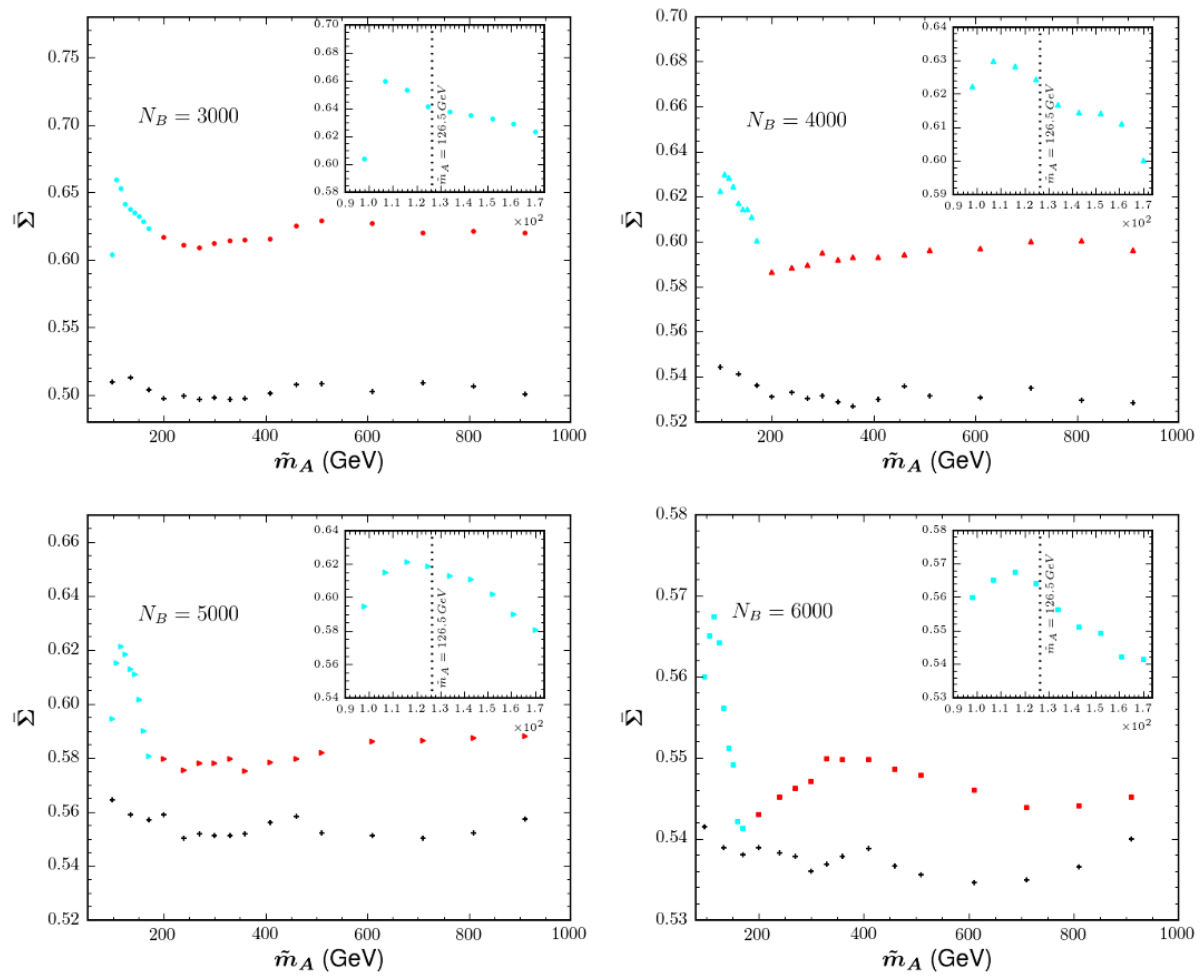

Figure 27. The same as figure 25, but accounting for the detector resolution as described in the text.

therefore $D$ pair-production is strong and may dominate the inclusive cross-section for $D$ production.

The presence of a second $D$ decay chain in the event can either be a blessing or a curse. If both $D$ particles decay the same way, as in figure 1, we can attempt to double our statistics by considering the second decay chain as well. However, this comes at a cost, since now we have to face the combinatorial problem of associating the different reconstructed final state objects to one of the two decay chains. First, there is a two-fold ambiguity of associating each jet to the correct side, and furthermore, there is an additional two-fold ambiguity in the case when all leptons are the same flavor. The simplest approach would be to consider all possible combinations and use the resulting data set for building the Voronoi tessellation, then proceeding with the fitting of the boundary surface as before. The result from this exercise is shown in figure 28 for the case of 100 (left panel) and 500 (right panel) signal events. We see that despite the pollution from wrong combinatorics, the peak in $\bar{\Sigma}$ is still very well visible, and found in the right location.

Our final study is reserved for the most challenging example so far - a case with a severe combinatorics problem, in the presence of SM $(t \bar{t})$ background events. For signal, let us again consider $D$-pair production, only this time let all three of the decay products of the second $D$ particle be QCD jets. Thus, each one of our signal events has 2 leptons and 4 jets, and picking the correct jet becomes a difficult task. Now, instead of using all possible combinations, we design a preselection cut in order to improve our chances of capturing 

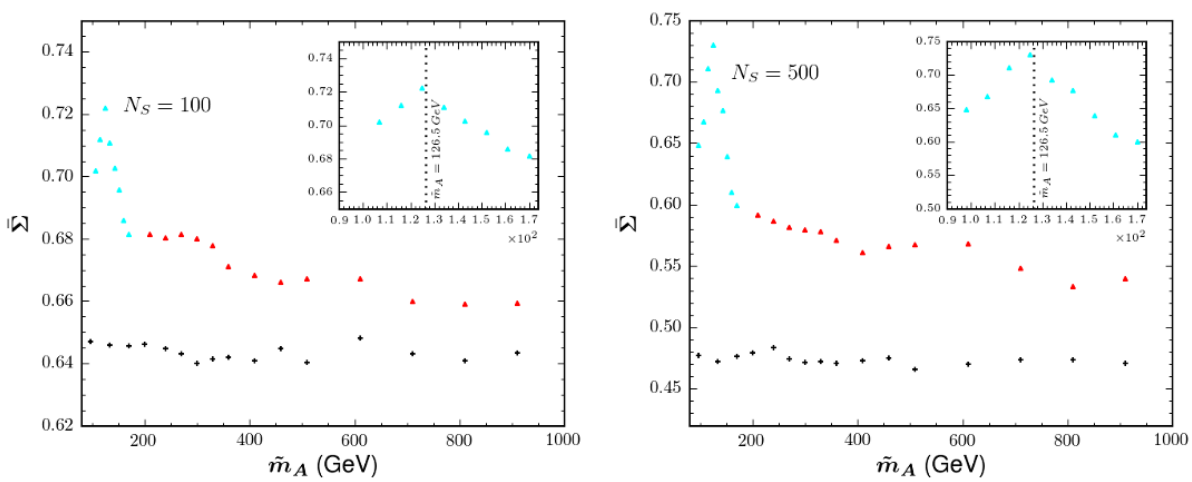

Figure 28. The same as figure 25, but for signal events where particles $D$ are pair-produced and decay as in figure 1. The left (right) panel is made with 100 (500) signal events.
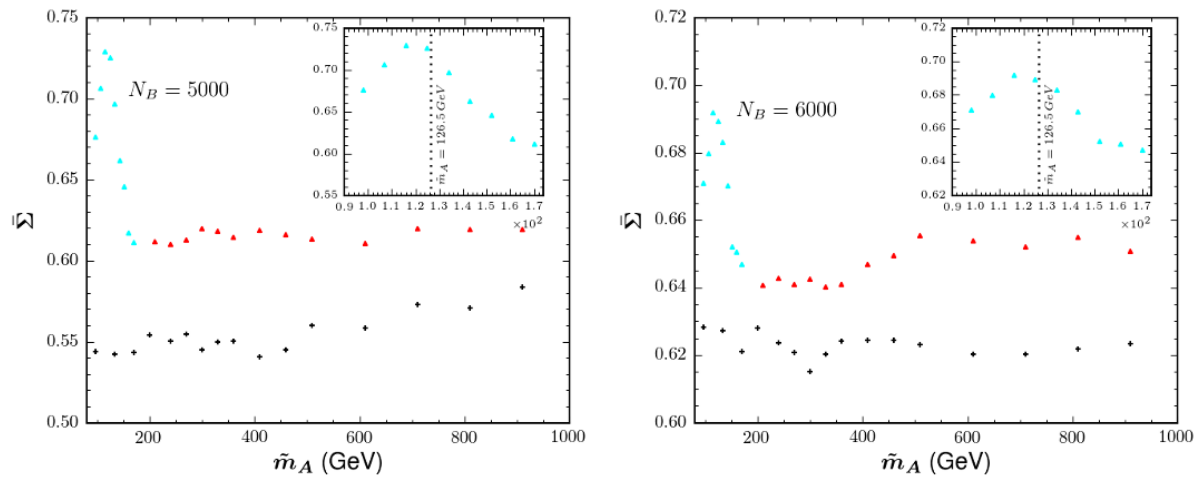

Figure 29. The same as the lower two plots in figure 25, but for signal events where particles $D$ are pair-produced and one of them decays as in figure 1 , while the other decays to 3 jets and particle A.

the correct jet pairing. For this purpose, we consider the four possible jet-lepton-lepton combinations and compute the corresponding three-body invariant masses. Next, we rankorder these four values $[17,18]$ and eliminate from further consideration the two jets which correspond to the two largest jet-lepton-lepton invariant masses, since those jets are very likely to come from the decay chain opposite the two leptons. The remaining two jets still cause a two-fold ambiguity, which we handle as in figure 28: by simply plotting both combinations. The end result from the analysis is shown in figure 29. As in the example from section 4.3, here we also include a certain number of $t \bar{t}$ background events: 5000 in the left panel and 6000 in the right panel. The fact that the $\bar{\Sigma}$ peak is again obtained in the correct location indicates that our method can be viable in the presence of combinatorial background due to pair production of particles $D$.

\section{Conclusions}

In this paper, we reconsidered the classic endpoint method for particle mass determination in SUSY-like decay chains like the one shown in figure 1. Our main points are: 
- We have identified a "flat direction" in the mass parameter space (1.1), along which mass differences can be measured relatively well, but the overall mass scale remains poorly constrained. (The analytical formulas parametrizing this flat direction can be found in appendix A.) We quantified the problem with examples of specific study points, $P_{31}$ and $P_{32}$, considered in sections 3 and 4 , respectively.

- We then proposed a new method for mass measurements in general, and for extracting the mass scale along the flat direction, in particular. The method takes advantage of the changes in the shape of the two-dimensional kinematic boundary surface within the fully differential three-dimensional space of observables, as one moves along the flat direction. We have tested our Voronoi-based algorithm [93, 95] for detecting the boundary surface and demonstrated that it can be usefully applied in order to lift the degeneracy along the flat direction. This approach represents the natural extension of the one-dimensional kinematic endpoint method to the relevant three dimensions of invariant mass phase space.

- We introduced a new variable, $\bar{\Sigma}$, which is the average RSD per unit area, calculated over the hypothesized kinematic boundary. We showed that the location of the $\bar{\Sigma}$ maximum correlates very well with the true values of the new particle masses, see figures $11,13,23,25$, and 27.

The work reported here can be extended in several directions. First of all, the method can be readily generalized to longer decay chains with more visible particles, where the boundary enhancement is even more pronounced [94], and therefore, the detection of the boundary surface should be in principle easier. One could also try to apply Voronoi-based boundary detection algorithms for the discovery of new physics. It is also interesting to develop a general and universal method for estimating the statistical significance of the local peaks found in figures 11, 13, 23 and 25, and hence the statistical precision of our mass measurement. These, along with many other interesting questions, will be investigated in future studies [109].

\section{Acknowledgments}

The research of the authors is supported by the National Science Foundation Grant Number PHY-1620610 and by the Department of Energy under Grants DE-SC0010296 and DESC0010504. DK is presently supported in part by the Korean Research Foundation (KRF) through the CERN-Korea Fellowship program, and also acknowledges support by the LHCTI postdoctoral fellowship under grant NSF-PHY-0969510.

\section{A Inverse formulas}

In this appendix we derive the inverse relations which define $m_{B}, m_{C}$ and $m_{D}$ in terms of the three measured endpoints

$$
a \equiv\left(m_{l l}^{\max }\right)^{2}, \quad b \equiv\left(m_{j \ell \ell}^{\max }\right)^{2}, \quad c \equiv\left(m_{j l(l o w)}^{\max }\right)^{2},
$$


and the remaining mass parameter $m_{A}$. For simplicity of notation, in this appendix we shall omit the tildes on the trial mass parameters $m_{A}, m_{B}, m_{C}$ and $m_{D}$.

The case of region $\mathbf{( 3 , 1 )}$. Region $(3,1)$ is defined by the following conditions

$$
\begin{aligned}
& R_{A B} \leq R_{B C} R_{C D}=R_{B D} \\
& R_{B C} \geq \frac{1}{2-R_{A B}}
\end{aligned}
$$

The kinematic endpoints are given by the following formulas:

$$
\begin{aligned}
a & =m_{D}^{2} R_{C D}\left(1-R_{A B}\right)\left(1-R_{B C}\right), \\
b & =m_{D}^{2}\left(1-R_{B D}\right)\left(1-R_{A B}\right), \\
c & =m_{D}^{2}\left(1-R_{B C}\right)\left(1-R_{C D}\right), \\
d & =m_{D}^{2}\left(1-R_{C D}\right)\left(1-R_{A B}\right) .
\end{aligned}
$$

The masses of $B, C$ and $D$ are given by

$$
\begin{aligned}
& m_{B}^{2}=\frac{1}{2 c}\left\{2 m_{A}^{2} c+a(b-a-c)+\left[4 m_{A}^{2} a c(b-a)+a^{2}(b-a-c)^{2}\right]^{1 / 2}\right\}, \\
& m_{C}^{2}=m_{B}^{2}\left(1+\frac{a}{m_{B}^{2}-m_{A}^{2}}\right), \\
& m_{D}^{2}=m_{B}^{2}\left(1+\frac{b}{m_{B}^{2}-m_{A}^{2}}\right),
\end{aligned}
$$

where in the right hand sides of the last two equations $m_{A}$ is an input, while $m_{B}$ is calculated from (A.8). Using (A.2) it is easy to show that in this region, we always have

$$
b-a-c=m_{D}^{2}\left(1-R_{C D}\right)\left(R_{B C}-R_{A B}\right) \geq 0,
$$

so that (A.8) always gives a non-negative result for $m_{B}^{2}$. Substituting (A.8)-(A.10) into (A.7), one can explicitly check that the $m_{A}$ dependence drops out and we recover the "bad" relation (2.12) in the form

$$
d=b-a
$$

The case of region $\mathbf{( 3 , 2 )}$. Region $(3,2)$ is defined by the following two conditions:

$$
\begin{aligned}
& R_{A B} \leq R_{B C} R_{C D}=R_{B D} \\
& R_{B C} \leq \frac{1}{2-R_{A B}}
\end{aligned}
$$

The kinematic endpoints are given by the following formulas:

$$
a=m_{C}^{2}\left(1-R_{A B}\right)\left(1-R_{B C}\right)
$$




$$
\begin{aligned}
& b=m_{D}^{2}\left(1-R_{A B}\right)\left(1-R_{B D}\right) \\
& c=m_{D}^{2}\left(1-R_{C D}\right)\left(1-R_{A B}\right)\left(2-R_{A B}\right)^{-1} \\
& d=m_{D}^{2}\left(1-R_{C D}\right)\left(1-R_{A B}\right) .
\end{aligned}
$$

The masses of $B, C$ and $D$ are given by [101]

$$
\begin{aligned}
& m_{B}^{2}=\frac{c m_{A}^{2}}{2 c-b+a}, \\
& m_{C}^{2}=m_{B}^{2}\left(1+\frac{a}{m_{B}^{2}-m_{A}^{2}}\right), \\
& m_{D}^{2}=m_{B}^{2}\left(1+\frac{b}{m_{B}^{2}-m_{A}^{2}}\right) .
\end{aligned}
$$

In this region, we always have

$$
2 c-b+a=m_{D}^{2}\left(1-R_{C D}\right)\left(\frac{2}{2-R_{A B}}-1\right) \geq 0,
$$

so that (A.18) always gives a non-negative result for $m_{B}^{2}$. The "bad" relation (2.12) is again satisfied in this region, so that along the flat direction the endpoint $d$ is again constant and given by (A.11), providing a useful cross-check on the obtained solution (A.18)-(A.20).

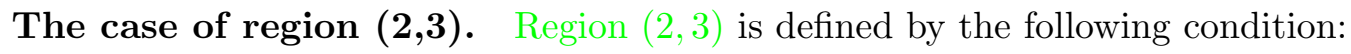

$$
R_{B C} \leq R_{A B} R_{C D}
$$

The kinematic endpoints are given by the following formulas:

$$
\begin{aligned}
& a=m_{C}^{2}\left(1-R_{A B}\right)\left(1-R_{B C}\right), \\
& b=m_{D}^{2}\left(1-R_{B C}\right)\left(1-R_{A B} R_{C D}\right), \\
& c=m_{D}^{2}\left(1-R_{C D}\right)\left(1-R_{A B}\right)\left(2-R_{A B}\right)^{-1}, \\
& d=m_{D}^{2}\left(1-R_{C D}\right)\left(1-R_{B C}\right) .
\end{aligned}
$$

The masses of $B, C$ and $D$ are given by

$$
\begin{aligned}
& m_{B}^{2}=\frac{2 m_{A}^{2}(b-a)+a(2 c-b+a)+\left[4 m_{A}^{2} a c(b-a)+a^{2}(2 c-b+a)^{2}\right]^{1 / 2}}{2(b-a)}, \\
& m_{C}^{2}=m_{B}^{2}\left(1+\frac{a}{m_{B}^{2}-m_{A}^{2}}\right) \\
& m_{D}^{2}=\left(1+\frac{a}{m_{B}^{2}-m_{A}^{2}}\right)\left[\frac{b}{a}\left(m_{B}^{2}-m_{A}^{2}\right)+m_{A}^{2}\right] .
\end{aligned}
$$

Using (A.22), (A.23) and (A.25) it is easy to show that in this region, we always have

$$
b-a=d \geq 0,
$$


and while the term $(2 c-b+a)$ can have either sign, the discriminant (i.e., the term inside the square root in (A.26)) is always larger than $a^{2}(2 c-b+a)^{2}$, which guarantees a nonnegative result for $m_{B}^{2}$. In this region, the relation (2.12) is again satisfied, so that $d$ is again given by (A.11), which can be used to cross-check the result (A.26)-(A.28).

The case of region $(\mathbf{4 , 1 )}$. Region $(4,1)$ is defined by the following conditions:

$$
\begin{aligned}
R_{A B} & \geq R_{B C} R_{C D}=R_{B D}, \\
R_{B C} & \geq \frac{1}{2-R_{A B}}, \\
R_{C D} & \geq R_{A B} R_{B C}=R_{A C} .
\end{aligned}
$$

For the case $(4,1)$ the endpoints are given by the following formulas:

$$
\begin{aligned}
& a=m_{C}^{2}\left(1-R_{A B}\right)\left(1-R_{B C}\right), \\
& b=m_{D}^{2}\left(1-\sqrt{R_{A D}}\right)^{2}, \\
& c=m_{D}^{2}\left(1-R_{C D}\right)\left(1-R_{B C}\right), \\
& d=m_{D}^{2}\left(1-R_{C D}\right)\left(1-R_{A B}\right) .
\end{aligned}
$$

The masses of $B, C$ and $D$ in terms of $a, b, c$ and $m_{A}$ are given by

$$
\begin{aligned}
& m_{B}^{2}=\frac{a m_{D}^{2}+c m_{A}^{2}+\left(m_{A}^{2}-a\right)(a+c)+a\left[\left(a+c-m_{A}^{2}-m_{D}^{2}\right)^{2}-4 m_{A}^{2} m_{D}^{2}\right]^{1 / 2}}{2(a+c)} \\
& m_{C}^{2}=m_{B}^{2}\left(1+\frac{a}{m_{B}^{2}-m_{A}^{2}}\right) \\
& m_{D}=m_{A}+\sqrt{b}
\end{aligned}
$$

where in (A.36) $m_{D}$ should be taken from (A.38), and the obtained result for $m_{B}$ should be used in (A.37). The $d$ endpoint is given by

$$
\begin{aligned}
d & =b-a-m_{D}^{2}\left(\sqrt{R_{A B}}-\sqrt{R_{B D}}\right)^{2} \\
& =\frac{a c\left\{(b-a-c)\left[b(1+\sqrt{r})+2 m_{A} \sqrt{b} \sqrt{r}\right]+2(2 b-a-c)\left(m_{A} \sqrt{b}+m_{A}^{2}\right)\right\}}{(a+c)\left[a(b-a-c)(1+\sqrt{r})+2 a m_{A} \sqrt{b}+2(a+c) m_{A}^{2}\right]},
\end{aligned}
$$

where

$$
r \equiv 1+\frac{4 m_{A}\left(m_{A}+\sqrt{b}\right)}{b-a-c} .
$$

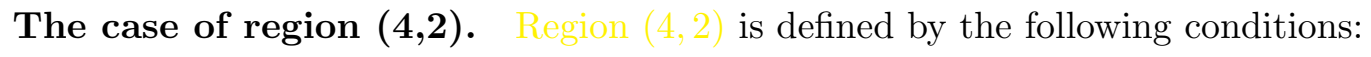

$$
\begin{aligned}
& R_{A B} \geq R_{B C} R_{C D}=R_{B D}, \\
& R_{B C} \geq R_{A B},
\end{aligned}
$$




$$
\begin{aligned}
& R_{B C} \leq \frac{1}{2-R_{A B}} \\
& R_{C D} \geq R_{A B} R_{B C}=R_{A C} .
\end{aligned}
$$

The kinematic endpoints are given by the following formulas:

$$
\begin{aligned}
& a=m_{C}^{2}\left(1-R_{A B}\right)\left(1-R_{B C}\right) \\
& b=m_{D}^{2}\left(1-\sqrt{R_{A D}}\right)^{2} \\
& c=m_{D}^{2}\left(1-R_{C D}\right)\left(1-R_{A B}\right)\left(2-R_{A B}\right)^{-1}, \\
& d=m_{D}^{2}\left(1-R_{C D}\right)\left(1-R_{A B}\right) .
\end{aligned}
$$

The masses of $B, C, D$ in terms of $a, b, c, m_{A}$ are the following

$$
\begin{aligned}
& m_{B}^{2}=\frac{1}{2}\left[m_{D}^{2}+m_{A}^{2}-a-2 c \pm \sqrt{\left(m_{D}^{2}+m_{A}^{2}-a-2 c\right)^{2}-4 m_{A}^{2}\left(m_{D}^{2}-c\right)}\right] \\
& m_{C}^{2}=m_{B}^{2}\left(1+\frac{a}{m_{B}^{2}-m_{A}^{2}}\right) \\
& m_{D}=m_{A}+\sqrt{b}
\end{aligned}
$$

Here, just as in (A.36)-(A.38), one should first find $m_{D}$ from (A.51), then use the result in (A.49) to obtain $m_{B}$, which will be needed in (A.50). The $d$ endpoint is then given by

$$
\begin{aligned}
d & =b-a-m_{D}^{2}\left(\sqrt{R_{A B}}-\sqrt{R_{B D}}\right)^{2} \\
& =\frac{c\left[a+3 b-2 c+6 m_{A} \sqrt{b}+2 m_{A}^{2}-\sqrt{\left(b-a-2 c+2 m_{A} \sqrt{b}\right)^{2}-4(a+c) m_{A}^{2}}\right]}{2\left(b-c+2 m_{A} \sqrt{b}+m_{A}^{2}\right)} .
\end{aligned}
$$

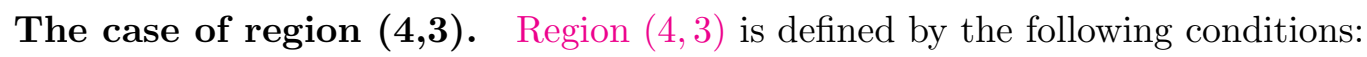

$$
\begin{aligned}
R_{A B} & \geq R_{B C}, \\
R_{B C} & \geq R_{A B} R_{C D}, \\
R_{C D} & \geq R_{A B} R_{B C}=R_{A C} .
\end{aligned}
$$

For the case $(4,3)$ the endpoints are given by the following formulas:

$$
\begin{aligned}
& a=m_{C}^{2}\left(1-R_{A B}\right)\left(1-R_{B C}\right) \\
& b=m_{D}^{2}\left(1-\sqrt{R_{A D}}\right)^{2} \\
& c=m_{D}^{2}\left(1-R_{C D}\right)\left(1-R_{A B}\right)\left(2-R_{A B}\right)^{-1}, \\
& d=m_{D}^{2}\left(1-R_{C D}\right)\left(1-R_{B C}\right) .
\end{aligned}
$$


The masses of $B, C, D$ in terms of $a, b, c, m_{A}$ are the following

$$
\begin{aligned}
& m_{B}^{2}=\frac{1}{2}\left[m_{D}^{2}+m_{A}^{2}-a-2 c-\sqrt{\left(m_{D}^{2}+m_{A}^{2}-a-2 c\right)^{2}-4 m_{A}^{2}\left(m_{D}^{2}-c\right)}\right], \\
& m_{C}^{2}=m_{B}^{2}\left(1+\frac{a}{m_{B}^{2}-m_{A}^{2}}\right), \\
& m_{D}=m_{A}+\sqrt{b}
\end{aligned}
$$

where again the masses are calculated in the order $m_{D}, m_{B}$ and then $m_{C}$. The $d$ endpoint is given by

$$
\begin{aligned}
d & =b-a-m_{D}^{2}\left(\sqrt{R_{B C}}-\sqrt{R_{A B} R_{C D}}\right)^{2} \\
& =\frac{4 a c\left[b-a-2 c+2 m_{A} \sqrt{b}+m_{A}^{2}-\sqrt{\left(b-a-2 c+2 m_{A} \sqrt{b}\right)^{2}-4(a+c) m_{A}^{2}}\right]}{\left(b-2 c+2 m_{A} \sqrt{b}-\sqrt{\left(b-a-2 c+2 m_{A} \sqrt{b}\right)^{2}-4(a+c) m_{A}^{2}}\right)^{2}-a^{2}} .
\end{aligned}
$$

Open Access. This article is distributed under the terms of the Creative Commons Attribution License (CC-BY 4.0), which permits any use, distribution and reproduction in any medium, provided the original author(s) and source are credited.

\section{References}

[1] P. Cushman et al., Working group report: WIMP dark matter direct detection, arXiv: 1310.8327 [INSPIRE].

[2] J. Buckley et al., Working group report: WIMP dark matter indirect detection, arXiv: 1310.7040 [INSPIRE].

[3] S. Arrenberg et al., Working group report: dark matter complementarity, arXiv:1310.8621 [INSPIRE].

[4] S.P. Martin, A Supersymmetry primer, Adv. Ser. Direct. High Energy Phys. 21 (2010) 1 [Adv. Ser. Direct. High Energy Phys. 18 (1998) 1] [hep-ph/9709356] [INSPIRE].

[5] T. Appelquist, H.-C. Cheng and B.A. Dobrescu, Bounds on universal extra dimensions, Phys. Rev. D 64 (2001) 035002 [hep-ph/0012100] [INSPIRE].

[6] H.-C. Cheng and I. Low, TeV symmetry and the little hierarchy problem, JHEP 09 (2003) 051 [hep-ph/0308199] [INSPIRE].

[7] D. Abercrombie et al., Dark matter benchmark models for early LHC run-2 searches: report of the ATLAS/CMS dark matter forum, arXiv:1507.00966 [INSPIRE].

[8] A.J. Barr and C.G. Lester, A review of the mass measurement techniques proposed for the Large Hadron Collider, J. Phys. G 37 (2010) 123001 [arXiv:1004.2732] [INSPIRE].

[9] W.S. Cho et al., On-shell constrained $M_{2}$ variables with applications to mass measurements and topology disambiguation, JHEP 08 (2014) 070 [arXiv:1401.1449] [INSPIRE].

[10] W.S. Cho, D. Kim, K.T. Matchev and M. Park, Probing resonance decays to two visible and multiple invisible particles, Phys. Rev. Lett. 112 (2014) 211801 [arXiv:1206.1546] [INSPIRE]. 
[11] F. Moortgat and L. Pape, CMS physics Technical Design Report. Volume 2, CERN-LHCC-2006 (2006), see chapter 13.4.

[12] S. Matsumoto, M.M. Nojiri and D. Nomura, Hunting for the top partner in the littlest Higgs model with T-parity at the CERN LHC, Phys. Rev. D 75 (2007) 055006 [hep-ph/0612249] [INSPIRE].

[13] A. Rajaraman and F. Yu, A new method for resolving combinatorial ambiguities at hadron colliders, Phys. Lett. B 700 (2011) 126 [arXiv:1009.2751] [INSPIRE].

[14] Y. Bai and H.-C. Cheng, Identifying dark matter event topologies at the LHC, JHEP 06 (2011) 021 [arXiv: 1012.1863] [INSPIRE].

[15] P. Baringer, K. Kong, M. McCaskey and D. Noonan, Revisiting combinatorial ambiguities at hadron colliders with $M_{T 2}$, JHEP 10 (2011) 101 [arXiv:1109.1563] [INSPIRE].

[16] K. Choi, D. Guadagnoli and C.B. Park, Reducing combinatorial uncertainties: a new technique based on MT2 variables, JHEP 11 (2011) 117 [arXiv:1109.2201] [INSPIRE].

[17] D. Kim, K.T. Matchev and M. Park, Using sorted invariant mass variables to evade combinatorial ambiguities in cascade decays, JHEP 02 (2016) 129 [arXiv: 1512.02222] [INSPIRE].

[18] M.D. Klimek, Ordered kinematic endpoints for 5-body cascade decays, JHEP 12 (2016) 132 [arXiv: 1610.08603] [INSPIRE].

[19] H.-C. Cheng, K.T. Matchev and M. Schmaltz, Bosonic supersymmetry? Getting fooled at the CERN LHC, Phys. Rev. D 66 (2002) 056006 [hep-ph/0205314] [InSPIRE].

[20] A. Freitas and D. Wyler, Phenomenology of mirror fermions in the littlest Higgs model with T-parity, JHEP 11 (2006) 061 [hep-ph/0609103] [INSPIRE].

[21] I. Hinchliffe and F.E. Paige, Measurements in gauge mediated SUSY breaking models at CERN LHC, Phys. Rev. D 60 (1999) 095002 [hep-ph/9812233] [INSPIRE].

[22] M.M. Nojiri, G. Polesello and D.R. Tovey, Proposal for a new reconstruction technique for SUSY processes at the LHC, hep-ph/0312317 [INSPIRE].

[23] K. Kawagoe, M.M. Nojiri and G. Polesello, A new SUSY mass reconstruction method at the CERN LHC, Phys. Rev. D 71 (2005) 035008 [hep-ph/0410160] [INSPIRE].

[24] H.-C. Cheng, J.F. Gunion, Z. Han, G. Marandella and B. McElrath, Mass determination in SUSY-like events with missing energy, JHEP 12 (2007) 076 [arXiv:0707.0030] [INSPIRE].

[25] M.M. Nojiri and M. Takeuchi, Study of the top reconstruction in top-partner events at the $L H C, J H E P 10$ (2008) 025 [arXiv:0802.4142] [INSPIRE].

[26] H.-C. Cheng, D. Engelhardt, J.F. Gunion, Z. Han and B. McElrath, Accurate mass determinations in decay chains with missing energy, Phys. Rev. Lett. 100 (2008) 252001 [arXiv:0802.4290] [INSPIRE].

[27] H.-C. Cheng, J.F. Gunion, Z. Han and B. McElrath, Accurate mass determinations in decay chains with missing energy. II, Phys. Rev. D 80 (2009) 035020 [arXiv:0905.1344] [INSPIRE].

[28] B. Webber, Mass determination in sequential particle decay chains, JHEP 09 (2009) 124 [arXiv: 0907.5307] [INSPIRE].

[29] C. Autermann, B. Mura, C. Sander, H. Schettler and P. Schleper, Determination of supersymmetric masses using kinematic fits at the LHC, arXiv:0911.2607 [INSPIRE]. 
[30] Z. Kang, N. Kersting, S. Kraml, A.R. Raklev and M.J. White, Neutralino reconstruction at the LHC from decay-frame kinematics, Eur. Phys. J. C 70 (2010) 271 [arXiv:0908.1550] [INSPIRE].

[31] M.M. Nojiri, K. Sakurai and B.R. Webber, Reconstructing particle masses from pairs of decay chains, JHEP 06 (2010) 069 [arXiv: 1005.2532] [INSPIRE].

[32] Z. Kang, N. Kersting and M. White, Mass estimation without using MET in early LHC data, arXiv: 1007.0382 [INSPIRE].

[33] J. Hubisz and J. Shao, Mass measurement in boosted decay chains with missing energy, Phys. Rev. D 84 (2011) 035031 [arXiv: 1009.1148] [INSPIRE].

[34] H.-C. Cheng, Z. Han, I.-W. Kim and L.-T. Wang, Missing momentum reconstruction and spin measurements at hadron colliders, JHEP 11 (2010) 122 [arXiv:1008.0405] [INSPIRE].

[35] B. Gripaios, K. Sakurai and B. Webber, Polynomials, Riemann surfaces and reconstructing missing-energy events, JHEP 09 (2011) 140 [arXiv:1103.3438] [INSPIRE].

[36] G.G. Ross and M. Serna, Mass determination of new states at hadron colliders, Phys. Lett. B 665 (2008) 212 [arXiv:0712.0943] [INSPIRE].

[37] A.J. Barr, G.G. Ross and M. Serna, The precision determination of invisible-particle masses at the LHC, Phys. Rev. D 78 (2008) 056006 [arXiv:0806.3224] [INSPIRE].

[38] A.J. Barr, A. Pinder and M. Serna, Precision determination of invisible-particle masses at the CERN LHC. II., Phys. Rev. D 79 (2009) 074005 [arXiv:0811.2138] [InSPIRE].

[39] P. Konar, K. Kong and K.T. Matchev, $\sqrt{\hat{s}}_{\text {min }}$ : a global inclusive variable for determining the mass scale of new physics in events with missing energy at hadron colliders, JHEP 03 (2009) 085 [arXiv: 0812.1042] [inSPIRE].

[40] P. Konar, K. Kong, K.T. Matchev and M. Park, RECO level $\sqrt{s}_{\text {min }}$ and subsystem $\sqrt{s}_{\text {min }}$ : Improved global inclusive variables for measuring the new physics mass scale in $E_{T}$ events at hadron colliders, JHEP 06 (2011) 041 [arXiv: 1006.0653] [INSPIRE].

[41] A.J. Barr et al., Guide to transverse projections and mass-constraining variables, Phys. Rev. D 84 (2011) 095031 [arXiv:1105.2977] [INSPIRE].

[42] T. Robens, $\sqrt{\hat{s}}_{\text {min }}$ resurrected, JHEP 02 (2012) 051 [arXiv: 1109.1018] [INSPIRE].

[43] R. Mahbubani, K.T. Matchev and M. Park, Re-interpreting the Oxbridge stransverse mass variable MT2 in general cases, JHEP 03 (2013) 134 [arXiv:1212.1720] [INSPIRE].

[44] Y. Bai, H.-C. Cheng, J. Gallicchio and J. Gu, A toolkit of the stop search via the chargino decay, JHEP 08 (2013) 085 [arXiv: 1304.3148] [INSPIRE].

[45] W.S. Cho et al., Improving the sensitivity of stop searches with on-shell constrained invariant mass variables, JHEP 05 (2015) 040 [arXiv: 1411.0664] [INSPIRE].

[46] W.S. Cho et al., OPTIMASS: a package for the minimization of kinematic mass functions with constraints, JHEP 01 (2016) 026 [arXiv: 1508.00589] [INSPIRE].

[47] P. Konar and A.K. Swain, Mass reconstruction with $M_{2}$ under constraint in semi-invisible production at a hadron collider, Phys. Rev. D 93 (2016) 015021 [arXiv:1509.00298] [INSPIRE].

[48] C.G. Lester and D.J. Summers, Measuring masses of semiinvisibly decaying particles pair produced at hadron colliders, Phys. Lett. B 463 (1999) 99 [hep-ph/9906349] [INSPIRE].

[49] A. Barr, C. Lester and P. Stephens, $m(T 2)$ : the truth behind the glamour, J. Phys. G 29 (2003) 2343 [hep-ph/0304226] [INSPIRE]. 
[50] M. Baumgart, T. Hartman, C. Kilic and L.-T. Wang, Discovery and measurement of sleptons, binos and winos with a Z-prime, JHEP 11 (2007) 084 [hep-ph/0608172] [INSPIRE].

[51] C. Lester and A. Barr, MTGEN: Mass scale measurements in pair-production at colliders, JHEP 12 (2007) 102 [arXiv:0708.1028] [INSPIRE].

[52] D.R. Tovey, On measuring the masses of pair-produced semi-invisibly decaying particles at hadron colliders, JHEP 04 (2008) 034 [arXiv:0802.2879] [INSPIRE].

[53] M. Serna, A short comparison between $m(T 2)$ and $m(C T)$, JHEP 06 (2008) 004 [arXiv: 0804.3344] [INSPIRE].

[54] M.M. Nojiri, K. Sakurai, Y. Shimizu and M. Takeuchi, Handling jets + missing E(T) channel using inclusive $m(T 2)$, JHEP 10 (2008) 100 [arXiv:0808.1094] [INSPIRE].

[55] W.S. Cho, K. Choi, Y.G. Kim and C.B. Park, M(T2)-assisted on-shell reconstruction of missing momenta and its application to spin measurement at the LHC, Phys. Rev. D 79 (2009) 031701 [arXiv:0810.4853] [inSPIRE].

[56] H.-C. Cheng and Z. Han, Minimal kinematic constraints and m(T2), JHEP 12 (2008) 063 [arXiv:0810.5178] [INSPIRE].

[57] M. Burns, K. Kong, K.T. Matchev and M. Park, Using subsystem MT2 for complete mass determinations in decay chains with missing energy at hadron colliders, JHEP 03 (2009) 143 [arXiv:0810.5576] [INSPIRE].

[58] K. Choi, S. Choi, J.S. Lee and C.B. Park, Reconstructing the Higgs boson in dileptonic $W$ decays at hadron collider, Phys. Rev. D 80 (2009) 073010 [arXiv:0908.0079] [INSPIRE].

[59] K.T. Matchev and M. Park, A General method for determining the masses of semi-invisibly decaying particles at hadron colliders, Phys. Rev. Lett. 107 (2011) 061801 [arXiv: 0910.1584] [INSPIRE].

[60] G. Polesello and D.R. Tovey, Supersymmetric particle mass measurement with the boost-corrected contransverse mass, JHEP 03 (2010) 030 [arXiv:0910.0174] [INSPIRE].

[61] P. Konar, K. Kong, K.T. Matchev and M. Park, Superpartner mass measurement technique using $1 D$ orthogonal decompositions of the Cambridge transverse mass variable $M_{T 2}$, Phys. Rev. Lett. 105 (2010) 051802 [arXiv:0910.3679] [INSPIRE].

[62] W.S. Cho, J.E. Kim and J.-H. Kim, Amplification of endpoint structure for new particle mass measurement at the LHC, Phys. Rev. D 81 (2010) 095010 [arXiv:0912.2354] [INSPIRE].

[63] M.M. Nojiri and K. Sakurai, Controlling ISR in sparticle mass reconstruction, Phys. Rev. D 82 (2010) 115026 [arXiv: 1008.1813] [INSPIRE].

[64] W.S. Cho, K. Choi, Y.G. Kim and C.B. Park, Gluino stransverse mass, Phys. Rev. Lett. 100 (2008) 171801 [arXiv: 0709.0288] [INSPIRE].

[65] B. Gripaios, Transverse observables and mass determination at hadron colliders, JHEP 02 (2008) 053 [arXiv: 0709.2740] [INSPIRE].

[66] A.J. Barr, B. Gripaios and C.G. Lester, Weighing wimps with kinks at colliders: invisible particle mass measurements from endpoints, JHEP 02 (2008) 014 [arXiv:0711.4008] [INSPIRE].

[67] W.S. Cho, K. Choi, Y.G. Kim and C.B. Park, Measuring superparticle masses at hadron collider using the transverse mass kink, JHEP 02 (2008) 035 [arXiv:0711.4526] [INSPIRE]. 
[68] M.M. Nojiri, Y. Shimizu, S. Okada and K. Kawagoe, Inclusive transverse mass analysis for squark and gluino mass determination, JHEP 06 (2008) 035 [arXiv:0802.2412] [INSPIRE].

[69] A.J. Barr, B. Gripaios and C.G. Lester, Transverse masses and kinematic constraints: from the boundary to the crease, JHEP 11 (2009) 096 [arXiv:0908.3779] [INSPIRE].

[70] K.T. Matchev, F. Moortgat, L. Pape and M. Park, Precision sparticle spectroscopy in the inclusive same-sign dilepton channel at LHC, Phys. Rev. D 82 (2010) 077701 [arXiv:0909.4300] [INSPIRE].

[71] P. Konar, K. Kong, K.T. Matchev and M. Park, Dark matter particle spectroscopy at the LHC: generalizing M(T2) to asymmetric event topologies, JHEP 04 (2010) 086 [arXiv: 0911.4126] [INSPIRE].

[72] K. Agashe, R. Franceschini and D. Kim, Simple "invariance" of two-body decay kinematics, Phys. Rev. D 88 (2013) 057701 [arXiv: 1209.0772] [inSPIRE].

[73] K. Agashe, R. Franceschini and D. Kim, Using energy peaks to measure new particle masses, JHEP 11 (2014) 059 [arXiv:1309.4776] [INSPIRE].

[74] K. Agashe, R. Franceschini, D. Kim and K. Wardlow, Mass measurement using energy spectra in three-body decays, JHEP 05 (2016) 138 [arXiv:1503.03836] [INSPIRE].

[75] K. Agashe, R. Franceschini, S. Hong and D. Kim, Energy spectra of massive two-body decay products and mass measurement, JHEP 04 (2016) 151 [arXiv: 1512.02265] [INSPIRE].

[76] K.T. Matchev, F. Moortgat, L. Pape and M. Park, Precise reconstruction of sparticle masses without ambiguities, JHEP 08 (2009) 104 [arXiv:0906.2417] [INSPIRE].

[77] I. Hinchliffe, F.E. Paige, M.D. Shapiro, J. Soderqvist and W. Yao, Precision SUSY measurements at CERN LHC, Phys. Rev. D 55 (1997) 5520 [hep-ph/9610544] [INSPIRE].

[78] H. Bachacou, I. Hinchliffe and F.E. Paige, Measurements of masses in SUGRA models at CERN LHC, Phys. Rev. D 62 (2000) 015009 [hep-ph/9907518] [INSPIRE].

[79] B.C. Allanach, C.G. Lester, M.A. Parker and B.R. Webber, Measuring sparticle masses in nonuniversal string inspired models at the LHC, JHEP 09 (2000) 004 [hep-ph/0007009] [INSPIRE].

[80] C.G. Lester, Model independent sparticle mass measurements at ATLAS, CERN-THESIS-2004-003 (2004).

[81] B.K. Gjelsten, D.J. Miller and P. Osland, Measurement of SUSY masses via cascade decays for SPS 1a, JHEP 12 (2004) 003 [hep-ph/0410303] [INSPIRE].

[82] B.K. Gjelsten, D.J. Miller and P. Osland, Measurement of the gluino mass via cascade decays for SPS 1a, JHEP 06 (2005) 015 [hep-ph/0501033] [INSPIRE].

[83] C.G. Lester, M.A. Parker and M.J. White, Determining SUSY model parameters and masses at the LHC using cross-sections, kinematic edges and other observables, JHEP 01 (2006) 080 [hep-ph/0508143] [INSPIRE].

[84] D.J. Miller, P. Osland and A.R. Raklev, Invariant mass distributions in cascade decays, JHEP 03 (2006) 034 [hep-ph/0510356] [INSPIRE].

[85] ATLAS collaboration, ATLAS detector and physics performance: technical design report, 2, CERN-LHCC-99-15 (1999).

[86] M.M. Nojiri, D. Toya and T. Kobayashi, Lepton energy asymmetry and precision SUSY study at hadron colliders, Phys. Rev. D 62 (2000) 075009 [hep-ph/0001267] [INSPIRE]. 
[87] C.G. Lester, Constrained invariant mass distributions in cascade decays: the shape of the 'm(qll)-threshold' and similar distributions, Phys. Lett. B 655 (2007) 39 [hep-ph/0603171] [INSPIRE].

[88] B.K. Gjelsten, D.J. Miller and P. Osland, Resolving ambiguities in mass determinations at future colliders, eConf C 050318 (2005) 0211 [hep-ph/0507232] [INSPIRE].

[89] M. Burns, K.T. Matchev and M. Park, Using kinematic boundary lines for particle mass measurements and disambiguation in SUSY-like events with missing energy, JHEP 05 (2009) 094 [arXiv:0903.4371] [INSPIRE].

[90] D. Costanzo and D.R. Tovey, Supersymmetric particle mass measurement with invariant mass correlations, JHEP 04 (2009) 084 [arXiv:0902.2331] [INSPIRE].

[91] C. Lester, Mass and spin measurement techniques (for the LHC), in The dark secrets of the Terascale, T. Tait and K. Matchev eds., World Scientific, Singapore (2013).

[92] P. Agrawal, C. Kilic, C. White and J.-H. Yu, Improved mass measurement using the boundary of many-body phase space, Phys. Rev. D 89 (2014) 015021 [arXiv:1308.6560] [INSPIRE].

[93] D. Debnath, J.S. Gainer, C. Kilic, D. Kim, K.T. Matchev and Y.-P. Yang, Identifying phase space boundaries with Voronoi tessellations, Eur. Phys. J. C 76 (2016) 645 [arXiv: 1606.02721] [INSPIRE].

[94] B. Altunkaynak, C. Kilic and M.D. Klimek, Multidimensional phase space methods for mass measurements and decay topology determination, Eur. Phys. J. C 77 (2017) 61 [arXiv: 1611.09764] [INSPIRE].

[95] D. Debnath, J.S. Gainer, D. Kim and K.T. Matchev, Edge detecting new physics the Voronoi way, Europhys. Lett. 114 (2016) 41001 [arXiv:1506.04141] [INSPIRE].

[96] S. Okabe, B. Boots and K. Sugihara, Spatial tessellations: concepts and applications of Voronoi diagrams, John Wiley \& Sons, U.S.A. (1992).

[97] C.B. Barber, D.P. Dobkin and H.T. Huhdanpaa, The Quickhull algorithm for convex hulls, ACM Trans. Math. Soft. 22 (1996) 469, see http://www.qhull.org.

[98] D. Debnath, J.S. Gainer, D. Kim and K.T. Matchev, Discovering new physics with Voronoi tessellations, arXiv:1511.02724 [INSPIRE].

[99] TGenPhaseSpace Class Reference, https://root.cern.ch/doc/v608/classTGenPhaseSpace.html.

[100] CMS collaboration, Measurement of masses in the $t \bar{t}$ system by kinematic endpoints in pp collisions at $\sqrt{s}=7$ TeV, Eur. Phys. J. C 73 (2013) 2494 [arXiv:1304.5783] [InSPIRE].

[101] B.K. Gjelsten, D.J. Miller, P. Osland and A.R. Raklev, Mass determination in cascade decays using shape formulas, AIP Conf. Proc. 903 (2007) 257 [hep-ph/0611259] [INSPIRE].

[102] J. Alwall et al., The automated computation of tree-level and next-to-leading order differential cross sections and their matching to parton shower simulations, JHEP 07 (2014) 079 [arXiv: 1405.0301] [INSPIRE].

[103] J. Reuter and D. Wiesler, Distorted mass edges at LHC from supersymmetric leptoquarks, Phys. Rev. D 84 (2011) 015012 [arXiv:1010.4215] [INSPIRE].

[104] B.C. Allanach, S. Grab and H.E. Haber, Supersymmetric Monojets at the Large Hadron Collider, JHEP 01 (2011) 138 [Erratum ibid. 1107 (2011) 087] [Erratum ibid. 09 (2011) 027] [arXiv: 1010.4261] [INSPIRE]. 
[105] C. Macesanu, C.D. McMullen and S. Nandi, Collider implications of universal extra dimensions, Phys. Rev. D 66 (2002) 015009 [hep-ph/0201300] [INSPIRE].

[106] M. ElKacimi, D. Goujdami, H. Przysiezniak and P.Z. Skands, One universal extra dimension in PYTHIA, Comput. Phys. Commun. 181 (2010) 122 [arXiv:0901.4087] [INSPIRE].

[107] J. Beuria, A. Datta, D. Debnath and K.T. Matchev, LHC collider phenomenology of minimal universal extra dimensions, arXiv:1702.00413 [INSPIRE].

[108] CMS collaboration, CMS physics: technical design report. Volume 1: detector performance and software, CERN-LHCC-2006-001 (2006).

[109] D. Debnath et al., Enhancing discovery sensitivity for SUSY-like events using kinematic boundary surfaces in phase space, in progress. 